\title{
Catalytic Enantioselective Synthesis of Amino Skipped Diynes
}

Paulo H. S. Paioti, ${ }^{\dagger}$ Khalil A. Abboud, ${ }^{\dagger}$ and Aaron Aponick*, ${ }^{+}$

${ }^{\dagger}$ Center for Heterocyclic Compounds, Department of Chemistry, University of Florida, Gainesville, Florida 32611, United States

${ }^{\ddagger}$ Center for X-Ray Crystallography, Department of Chemistry, University of Florida, Gainesville, Florida 32611, United States

\section{Supporting Information}

1. General Considerations.

2. Synthesis of Aldehydes, Alkynes and Amine Starting Materials.......................................... S3

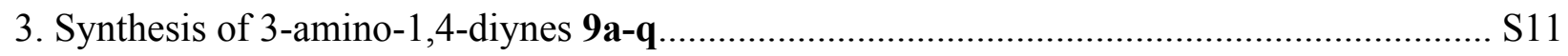

4. Synthesis of Compounds 10, 11, 13, 14, 15, 16, 17 and 4 ............................................ S29

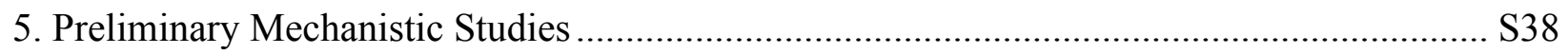

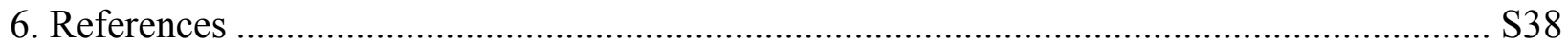

7. NMR Spectra of Aldehydes, Alkynes and Amine Starting Materials ................................... S41

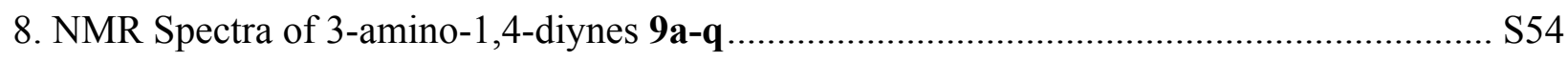

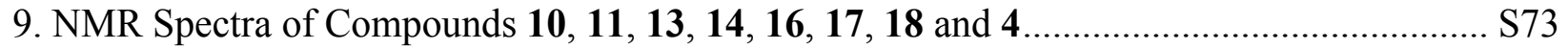

10. X-Ray Crystallographic Data of Compound ent-9b....................................................... S82 


\section{General Considerations}

All reactions were carried out under an atmosphere of nitrogen unless otherwise specified. Anhydrous solvents were transferred via syringe to flame-dried glassware, which had been cooled under a stream of dry nitrogen. Anhydrous tetrahydrofuran (THF), acetonitrile, diethyl ether, dichloromethane, toluene, benzene and pentane were dried using an mBraun solvent purification system. Analytical thin layer chromatography (TLC) was performed using $250 \mu \mathrm{m}$ Silica Gel 60 F254 pre-coated plates. Flash column chromatography was performed using 230-400 Mesh 60A Silica Gel. Proton nuclear magnetic resonance $\left({ }^{1} \mathrm{H}\right.$ NMR) spectra were recorded using Varian Unity Inova $500 \mathrm{MHz}$ and Varian Mercury $300 \mathrm{MHz}$ spectrometers. Chemical shifts $(\delta)$ are reported in parts per million (ppm) downfield relative to tetramethylsilane (TMS, $0.0 \mathrm{ppm}$ ) or $\mathrm{CDCl}_{3}(7.27$ ppm). Coupling constants $(J)$ are reported in Hz. Multiplicities are reported using the following abbreviations: s, singlet; d, doublet; t, triplet; q, quartet; p, pentet; m, multiplet; b, broad; Carbon-13 nuclear magnetic resonance $\left({ }^{13} \mathrm{C}\right.$ NMR) spectra were recorded using a Varian Unity Mercury 300 spectrometer at $75 \mathrm{MHz}$ and a Varian Unity Inova $500 \mathrm{MHz}$ at $125 \mathrm{MHz}$. Chemical shifts are reported in $\mathrm{ppm}$ relative to the carbon resonance of $\mathrm{CDCl}_{3}$ (77.23 ppm). Fluorine-19 nuclear magnetic resonance $\left({ }^{19} \mathrm{~F}\right.$ NMR) spectra were recorded using a Varian Unity Mercury 300 spectrometer at $282 \mathrm{MHz}$. Specific Optical rotations were obtained on a JASCD P - 2000 Series Polarimeter (wavelength $=589 \mathrm{~nm}$ ). High resolution mass spectra $($ HRMS) were obtained by Mass Spectrometry Core Laboratory of University of Florida, and are reported as $\mathrm{m} / \mathrm{z}$ (relative ratio). The Instrument Agilent 6200 was used for ESI-TOF analysis and an IonSense DART ET-100 ionization source was coupled to the instrument for DART-TOF analysis. Accurate $\mathrm{m} / \mathrm{z}$ are reported for the molecular ion $[\mathrm{M}+\mathrm{H}]^{+}$or $[\mathrm{M}+\mathrm{Na}]^{+}$. Enantiomeric ratios were determined by chiral HPLC analysis (Shimadzu) using Chiralpak IA, Chiralcel OD-H and OJ-H columns. Phenylacetylene, TMSacetylene, TIPS-acetylene, pent-1-yne, methylpropiolate, 2-iodoaniline, 4-bromoiodoaniline, 4iodoanisole, 1-bromo-4-iodobenzene, 1-fluoro-4-iodobenzene, dibenzylamine, morpholine, 4piperidone hydrate hydrochloride were purchased from Sigma-Aldrich. All purchased reagents were used without further purification. 


\section{Synthesis of Aldehydes, Alkynes and Amine Starting Materials}

2.1 Representative Procedure for the Preparation of Ynals 6a, 6e and $\mathbf{6 f}$

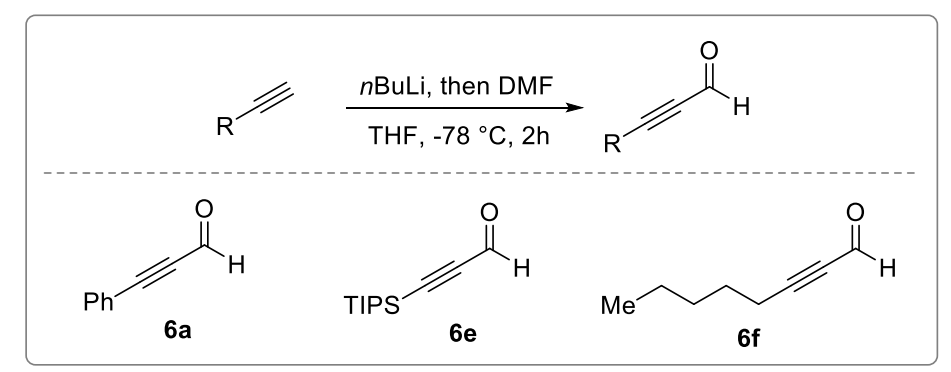

Adapting a literature procedure, ${ }^{1}$ an $n \mathrm{BuLi}$ solution $(2.0 \mathrm{M}$ in hexanes, $3.64 \mathrm{~mL}, 9.105$ mmol, 1.0 equiv) was added to a stirred solution of phenylacetylene $(0.930 \mathrm{~g}, 9.105 \mathrm{mmol}, 1.0$ equiv) in THF $(25 \mathrm{~mL})$ at $-78^{\circ} \mathrm{C}$. The mixture was stirred for 30 minutes then removed from the dry ice/acetone bath and stirred for 10 minutes before returning to the $-78^{\circ} \mathrm{C}$ bath. $\mathrm{DMF}(1.40$ $\mathrm{mL}, 18.210,2.0$ equiv) was then added to the flask and the reaction was stirred at $-78{ }^{\circ} \mathrm{C}$ for 10 minutes, warmed to room temperature and allowed to stir for 45 additional minutes. To quench the reaction, the mixture was poured into a stirred solution of $10 \%$ aqueous solution of $\mathrm{KH}_{2} \mathrm{PO}_{4}$ $(100 \mathrm{~mL})$ and $\mathrm{Et}_{2} \mathrm{O}(100 \mathrm{~mL})$ at $0^{\circ} \mathrm{C}$ and stirred for $5 \mathrm{~min}$, at which point the layers were separated and the organic layer was washed with water $(50 \mathrm{~mL})$, dried over MgSO4, filtered, and concentrated under reduced pressure to give a residue which was purified by flash column chromatography on silica gel (0-5\% EtOAc/hexanes gradient) to afford the desired propargyl aldehyde $6 \mathbf{a}$ in $75 \%$ yield $(0.889 \mathrm{~g}, 8.092 \mathrm{mmol})$ as a yellowish oil.

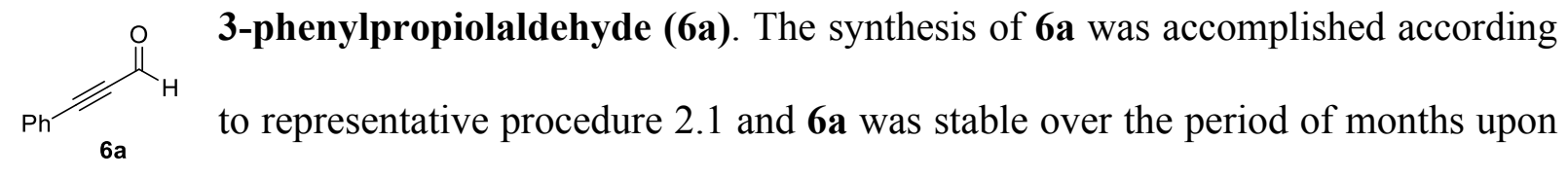

storage at $-5^{\circ} \mathrm{C}$. The spectroscopic data matched the reported data for this compound. ${ }^{1}$ Compound 6a was obtained as a yellowish oil in $75 \%$ yield $(0.889 \mathrm{~g}, 8.092 \mathrm{mmol}) . \mathrm{R}_{f}=0.47$ 
(10\% EtOAc in hexanes). ${ }^{1} \mathrm{H}$ NMR $\left(500 \mathrm{MHz}, \mathrm{CDCl}_{3}\right) \delta 9.44(\mathrm{~s}, 1 \mathrm{H}), 7.66-7.57(\mathrm{~m}, 2 \mathrm{H}), 7.53$

$-7.48(\mathrm{~m}, 1 \mathrm{H}), 7.45-7.39(\mathrm{~m}, 2 \mathrm{H}) .{ }^{13} \mathrm{C} \mathrm{NMR}\left(125 \mathrm{MHz}, \mathrm{CDCl}_{3}\right) \delta 176.9,133.4,131.5,128.9$, 119.6, 95.3, 88.6.

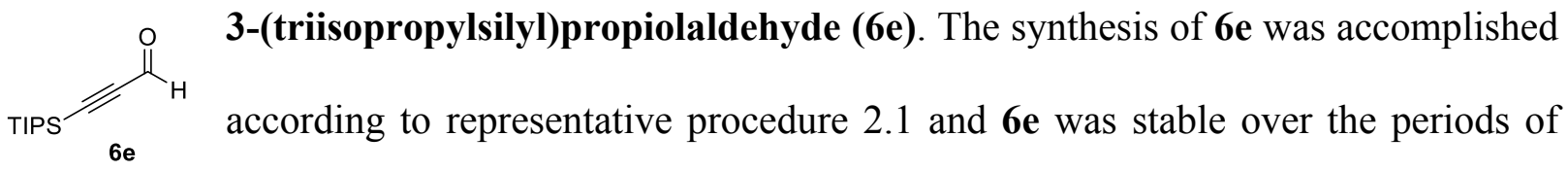
months upon storage at $-5^{\circ} \mathrm{C}$. The spectroscopic data matched the reported data for this compound. ${ }^{2}$ Compound $6 \mathrm{e}$ was obtained as a colorless oil in $78 \%$ yield $(1.475 \mathrm{~g}, 7.023 \mathrm{mmol})$. $\mathrm{R}_{f}=0.70\left(10 \%\right.$ EtOAc in hexanes). ${ }^{1} \mathrm{H}$ NMR $\left(500 \mathrm{MHz}, \mathrm{CDCl}_{3}\right) \delta 9.21(\mathrm{~s}, 1 \mathrm{H}), 1.12(\mathrm{~s}, 18 \mathrm{H})$, $1.12-1.10(\mathrm{~m}, 3 \mathrm{H}) .{ }^{13} \mathrm{C}$ NMR $\left(125 \mathrm{MHz}, \mathrm{CDCl}_{3}\right) \delta 176.7,104.7,100.9,18.6,11.2$.

representative procedure 2.1 and $\mathbf{6 f}$ was stable over the periods of weeks upon storage at $-5^{\circ} \mathrm{C}$. The spectroscopic data matched the reported data for this compound. ${ }^{3}$ Compound $6 \mathbf{f}$ was obtained as a colorless oil in $86 \%$ yield $(1.627 \mathrm{~g}, 13.102 \mathrm{mmol}) . \mathrm{R}_{f}=0.60$ (10\% EtOAc in hexanes). ${ }^{1} \mathrm{H}$ NMR $\left(500 \mathrm{MHz}, \mathrm{CDCl}_{3}\right) \delta 9.19(\mathrm{~s}, 1 \mathrm{H}), 2.42(\mathrm{~s}, 2 \mathrm{H}), 1.72-1.50$ (m, 2H), $1.52-1.24(\mathrm{~m}, 4 \mathrm{H}), 0.92(\mathrm{td}, J=7.2,1.4 \mathrm{~Hz}, 3 \mathrm{H})$.

2.2 Representative Procedure for the Preparation of Ynals $\mathbf{6 b}, \mathbf{6 c}$ and $\mathbf{6 d}$

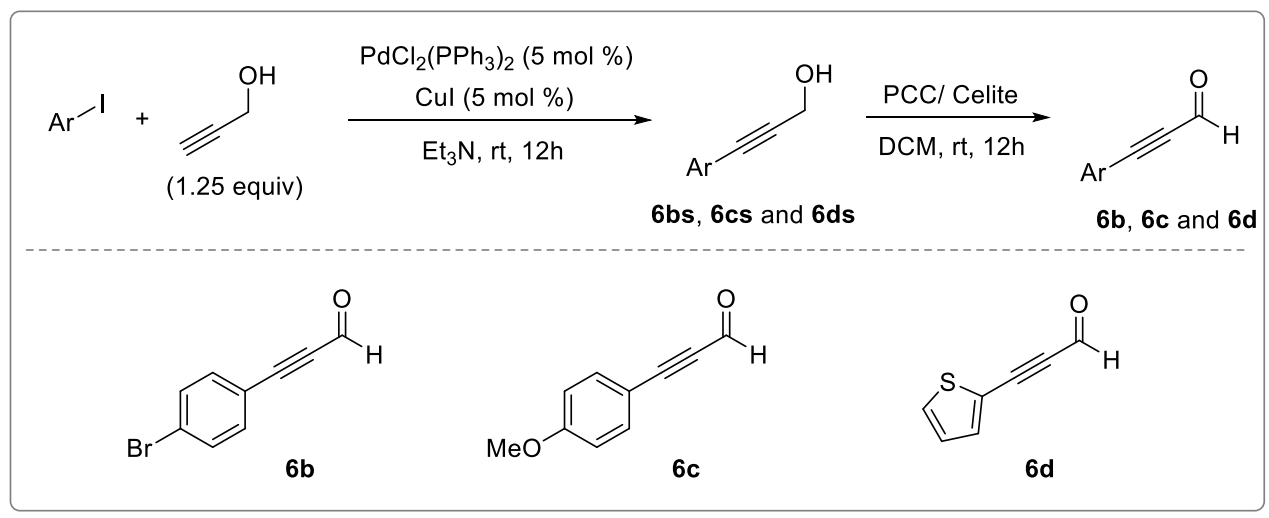


Adapting a literature procedure, ${ }^{1} \mathrm{CuI}(47.6 \mathrm{mg}, 0.25 \mathrm{mmol}, 5 \mathrm{~mol} \%), \mathrm{PdCl}_{2}\left(\mathrm{PPh}_{3}\right)_{2}$ (175.5 mg, $0.25 \mathrm{mmol}, 5 \mathrm{~mol} \%$ ) and propargyl alcohol ( $0.37 \mathrm{~mL}, 6.25 \mathrm{mmol}, 1.25$ equiv) were added to a stirred solution of 1-bromo-4-iodobenzene (1.415 g, $5.000 \mathrm{mmol}, 1.0$ equiv) in $\mathrm{Et}_{3} \mathrm{~N}$ (13.9 $\mathrm{mL}, 100 \mathrm{mmol}, 20$ equiv) at room temperature. The mixture was stirred for $12 \mathrm{~h}$, concentrated under reduced pressure, and purified by flash colum chromatography on silica gel (40\% EtOAc in hexanes) to afford the 3-(4-bromophenyl)prop-2-yn-1-ol (0.988 g, $4.70 \mathrm{mmol})$ in $94 \%$ yield as a yellow solid that was taken to on to the next step of the sequence.

Following a related literature procedure, ${ }^{4}$ PCC (0.689 g, $3.198 \mathrm{mmol}, 1.5$ equiv) and celite $(0.689 \mathrm{~g})$ were added to a stirred solution of 3-(4-bromophenyl)prop-2-yn-1-ol (0.450 g, $2.132 \mathrm{mmol}, 1.0$ equiv) in dichloromethane $(20 \mathrm{~mL})$, at room temperature. The mixture was stirred for $12 \mathrm{~h}$ and directly submitted to flash column chromatography on silica gel (10\% EtOAc in hexanes) to afford the 3-(4-bromophenyl)propiolaldehyde $\mathbf{6 b}(0.272 \mathrm{~g}, 1.30 \mathrm{mmol})$ in $61 \%$ yield as a yellow solid.

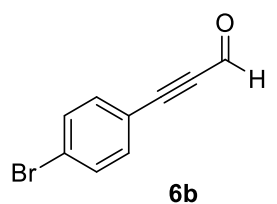

3-(4-bromophenyl)propiolaldehyde (6b). The synthesis of alcohol 6 bs was accomplished according to representative procedure 2.2. The spectroscopic data matched the reported data for this compound. ${ }^{4}$ Compound $6 \mathbf{b s}$ was obtained as a yellow solid in $94 \%$ yield $(0.988 \mathrm{~g}, 4.70 \mathrm{mmol}) . \mathrm{R}_{f}=0.09(20 \%$ EtOAc in hexanes). ${ }^{1} \mathrm{H}$ NMR $\left(500 \mathrm{MHz}, \mathrm{CDCl}_{3}\right) \delta 7.43(\mathrm{~d}, J=8.5 \mathrm{~Hz}, 2 \mathrm{H}), 7.27(\mathrm{~d}, J=8.5 \mathrm{~Hz}, 2 \mathrm{H}), 4.48$ (s, 2H), $2.12(\mathrm{bs}, 1 \mathrm{H}) .{ }^{13} \mathrm{C}$ NMR $\left(125 \mathrm{MHz}, \mathrm{CDCl}_{3}\right) \delta 133.3,131.8,123.0,121.6,88.6,84.8$, 51.7.

The synthesis of $\mathbf{6 b}$ was accomplished according to representative procedure 2.2 and $\mathbf{6 b}$ was stable over the periods of months upon storage at $-5^{\circ} \mathrm{C}$. The spectroscopic data matched the reported data for this compound. ${ }^{1}$ Compound $\mathbf{6 b}$ was obtained as a yellow solid in $61 \%$ yield 
(0.272 g, $1.30 \mathrm{mmol}) . \mathrm{R}_{f}=0.56\left(20 \%\right.$ EtOAc in hexanes). ${ }^{1} \mathrm{H} \mathrm{NMR}\left(500 \mathrm{MHz}, \mathrm{CDCl}_{3}\right) \delta 9.37(\mathrm{~s}$, $1 \mathrm{H}), 7.52(\mathrm{~d}, J=8.4 \mathrm{~Hz}, 2 \mathrm{H}), 7.42(\mathrm{~d}, J=8.4 \mathrm{~Hz}, 2 \mathrm{H}) .{ }^{13} \mathrm{C} \mathrm{NMR}\left(125 \mathrm{MHz}, \mathrm{CDCl}_{3}\right) \delta 176.7,134.7$, 132.4, 126.4, 118.5, 93.8, 89.3.

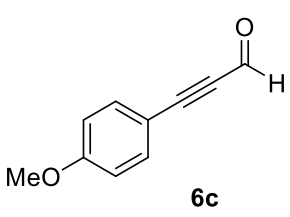

3-(4-methoxyphenyl)propiolaldehyde (6c). The synthesis of alcohol 6cs was accomplished according to representative procedure 2.2. The spectroscopic data matched the reported data for this compound. ${ }^{4}$ Compound 6cs was obtained as a yellow solid in $85 \%$ yield $(1.051 \mathrm{~g}, 6.48 \mathrm{mmol}) . \mathrm{R}_{f}=0.20$ (20\% EtOAc in hexanes). ${ }^{1} \mathrm{H}$ NMR $\left(500 \mathrm{MHz}, \mathrm{CDCl}_{3}\right) \delta 7.38(\mathrm{~d}, J=8.8 \mathrm{~Hz}, 2 \mathrm{H}), 6.85(\mathrm{~d}, J=8.8 \mathrm{~Hz}, 2 \mathrm{H}), 4.49$ $(\mathrm{d}, J=4.4 \mathrm{~Hz}, 2 \mathrm{H}), 3.82(\mathrm{~s}, 3 \mathrm{H}), 1.77(\mathrm{bs}, 1 \mathrm{H}) .{ }^{13} \mathrm{C} \mathrm{NMR}\left(125 \mathrm{MHz}, \mathrm{CDCl}_{3}\right) \delta 160.0,133.4$, $114.8,114.2,86.1,85.9,55.5,51.9$.

The synthesis of $\mathbf{6 c}$ was accomplished according to representative procedure 2.2 and $\mathbf{6 c}$ was stable over the period of months upon storage at $-5^{\circ} \mathrm{C}$. The spectroscopic data matched the reported data for this compound. ${ }^{1}$ Compound $\mathbf{6 c}$ was obtained as a yellow solid in $41 \%$ yield $(0.194 \mathrm{~g}, 1.21 \mathrm{mmol}) . \mathrm{R}_{f}=0.56\left(20 \%\right.$ EtOAc in hexanes). ${ }^{1} \mathrm{H} \mathrm{NMR}\left(500 \mathrm{MHz}, \mathrm{CDCl}_{3}\right) \delta 9.37(\mathrm{~s}$, $1 \mathrm{H}), 7.53(\mathrm{dd}, J=7.0,2.0 \mathrm{~Hz}, 2 \mathrm{H}), 6.89(\mathrm{dd}, J=7.0,2.0 \mathrm{~Hz}, 2 \mathrm{H}), 3.82(\mathrm{~s}, 3 \mathrm{H}) .{ }^{13} \mathrm{C} \mathrm{NMR}$ $\left(125 \mathrm{MHz}, \mathrm{CDCl}_{3}\right) \delta 176.8,162.3,135.5,114.6,111.2,96.7,88.9,55.6$.

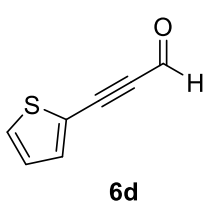

3-(thiophen-2-yl)propiolaldehyde (6d). The synthesis of alcohol 6ds was accomplished according to representative procedure 2.2. The compound $\mathbf{6 d s}$ was obtained as an orange solid in $82 \%$ yield $(1.108 \mathrm{~g}, 8.02 \mathrm{mmol}) . \mathrm{R}_{f}=0.20(20 \%$ EtOAc in hexanes) ${ }^{1} \mathrm{H}$ NMR $\left(500 \mathrm{MHz}, \mathrm{CDCl}_{3}\right) \delta 7.28(\mathrm{dd}, \mathrm{J}=5.2,1.2 \mathrm{~Hz}, 1 \mathrm{H}), 7.24(\mathrm{dd}, J=$ 3.6, $1.2 \mathrm{~Hz}, 1 \mathrm{H}), 6.99(\mathrm{dd}, J=5.2,3.6 \mathrm{~Hz}, 1 \mathrm{H}), 4.53(\mathrm{~s}, 2 \mathrm{H}), 2.25(\mathrm{bd}, J=23.2 \mathrm{~Hz}, 1 \mathrm{H}) .{ }^{13} \mathrm{C}$ NMR (125MHz, $\left.\mathrm{CDCl}_{3}\right) \delta 132.6,127.6,127.1,122.6,91.4,79.2,51.8$. 
The synthesis of $\mathbf{6 d}$ was accomplished according to representative procedure 2.2 and $\mathbf{6 d}$ was stored at $-5^{\circ} \mathrm{C}$. The spectroscopic data collected matched the reported data for this compound. ${ }^{1}$ Compound $\mathbf{6 d}$ was obtained as a yellow solid in $37 \%$ yield $(0.219,1.61 \mathrm{mmol}) . \mathrm{R}_{f}=$ $0.50\left(20 \%\right.$ EtOAc in hexanes). ${ }^{1} \mathrm{H}$ NMR $\left(500 \mathrm{MHz}, \mathrm{CDCl}_{3}\right) \delta 9.41(\mathrm{~d}, J=0.7 \mathrm{~Hz}, 1 \mathrm{H}), 7.68-$ $7.44(\mathrm{~m}, 2 \mathrm{H}), 7.11(\mathrm{ddd}, J=5.0,3.7,0.7 \mathrm{~Hz}, 1 \mathrm{H}) .{ }^{13} \mathrm{C} \mathrm{NMR}\left(125 \mathrm{MHz}, \mathrm{CDCl}_{3}\right) \delta$ 176.2, 137.7, $132.8,128.1,119.4,93.4,89.4$.

2.3 Procedure for the Preparation of Propargyl Aldehyde $\mathbf{6 g}$

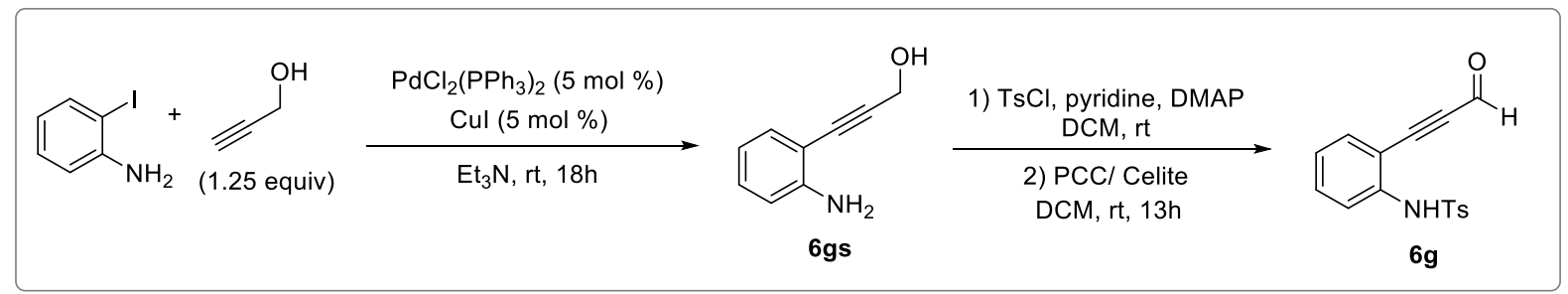

3-(2-aminophenyl)prop-2-yn-1-ol (6gs). Adapting a literature procedure, ${ }^{1} \mathrm{CuI}(43.5 \mathrm{mg}$, $0.23 \mathrm{mmol}, 5 \mathrm{~mol} \%), \mathrm{PdCl}_{2}\left(\mathrm{PPh}_{3}\right)_{2}(160.2 \mathrm{mg}, 0.23 \mathrm{mmol}, 5 \mathrm{~mol} \%)$ and propargyl alcohol ( $0.34 \mathrm{~mL}, 5.70 \mathrm{mmol}, 1.25$ equiv) were added to a stirred solution of 2-iodoaniline (1.00 g, $4.566 \mathrm{mmol}, 1.0$ equiv) in $\mathrm{Et}_{3} \mathrm{~N}(12.7 \mathrm{~mL}, 91.32 \mathrm{mmol}, 20$ equiv), at room temperature. The mixture was allowed to react for $18 \mathrm{~h}$, concentrated under reduced pressure, and submitted to flash colum chromatography on silica gel (40\% EtOAc in hexanes) to afford the compound $\mathbf{6 g s}$ $(0.416 \mathrm{~g}, 2.83 \mathrm{mmol})$ in $61 \%$ yield as a orange solid. $\mathrm{R}_{f}=0.23$ (40\% EtOAc in hexanes). ${ }^{1} \mathrm{H}$ $\operatorname{NMR}\left(500 \mathrm{MHz}, \mathrm{CDCl}_{3}\right) \delta 7.29(\mathrm{dd}, J=8.3,1.5 \mathrm{~Hz}, 1 \mathrm{H}), 7.16(\mathrm{td}, J=7.8,1.5 \mathrm{~Hz}, 1 \mathrm{H}), 6.72(\mathrm{~d}$, $\mathrm{J}=7.8 \mathrm{~Hz}, 2 \mathrm{H}), 4.55(\mathrm{~s}, 2 \mathrm{H}), 4.30(\mathrm{~s}, 1 \mathrm{H}), 4.28(\mathrm{bs}, 2 \mathrm{H}) .{ }^{13} \mathrm{C} \mathrm{NMR}\left(125 \mathrm{MHz}, \mathrm{CDCl}_{3}\right)$ $\delta$ 148.1, 132.5, 130.1, 118.2, 114.7, 107.5, 93.0, 82.4, 51.8. 
4-methyl- $N$-(2-(3-oxoprop-1-yn-1-yl)phenyl)benzenesulfonamide (6g). Adapting a literature procedure ${ }^{4}$ pyridine $(0.27 \mathrm{~mL}, 3.28 \mathrm{mmol}, 3.0$ equiv $)$, catalytic DMAP (1 crystal) and $\mathrm{TsCl}(0.209 \mathrm{~g}, 1.094 \mathrm{mmol}, 1.0$ equiv) were added to a solution of $\mathbf{6 g s}$ obtained above $(0.161 \mathrm{~g}$, $1.094 \mathrm{mmol})$ in dichloromethane $(4.0 \mathrm{~mL})$ at room temperature and the mixture was stirred for $16 \mathrm{~h}$. The reaction was then diluted with dichloromethane $(4.0 \mathrm{~mL})$ and quenched with a saturated solution of $\mathrm{NH}_{4} \mathrm{Cl}(4.0 \mathrm{~mL})$. The organic layer was separated, dried over $\mathrm{MgSO}_{4}$, concentrated, and subjected to flash column chromatography on silica gel $(20-40 \%$ EtOAc in hexanes, gradient) to furnish compound the sulfonamide as a white solid, which was taken directly to the next step, in 55\% yield $(0.181 \mathrm{~g}, 0.602 \mathrm{mmol})$. PCC $(0.0778 \mathrm{~g}, 0.361 \mathrm{mmol}, 1.5$ equiv) and celite (0.0778 g) were added to a stirred solution of $N$-(2-(3-hydroxyprop-1-yn-1yl)phenyl)-4-methylbenzenesulfonamide thus obtained $(0.0725 \mathrm{~g}, 0.240 \mathrm{mmol}, 1.0 \mathrm{equiv})$ in dichloromethane $(2.5 \mathrm{~mL})$, at room temperature. The mixture was allowed to react for $13 \mathrm{~h}$ then directly subjected to flash column chromatography on silica gel (20-40\% EtOAc in hexanes, gradient) to afford compound $\mathbf{6 g}(0.0321 \mathrm{~g}, 0.107 \mathrm{mmol})$ in $45 \%$ yield as a slightly red solid. The compound $6 \mathrm{~g}$ was stored at $-5^{\circ} \mathrm{C}$ and used freshly. $\mathrm{R}_{f}=0.69$ (40\% EtOAc in hexanes). ${ }^{1} \mathrm{H}$ NMR $\left(500 \mathrm{MHz}, \mathrm{CDCl}_{3}\right) \delta 9.36(\mathrm{~s}, 1 \mathrm{H}), 7.70(\mathrm{~d}, J=8.2 \mathrm{~Hz}, 2 \mathrm{H}), 7.65(\mathrm{~d}, \mathrm{~J}=8.0 \mathrm{~Hz}, 2 \mathrm{H}), 7.45$ (ddt, $J=7.5,4.8,2.4 \mathrm{~Hz}, 2 \mathrm{H}), 7.32(\mathrm{~s}, 1 \mathrm{H}), 7.23(\mathrm{~d}, J=8.0 \mathrm{~Hz}, 2 \mathrm{H}), 7.12(\mathrm{t}, J=7.5 \mathrm{~Hz}, 1 \mathrm{H})$, $2.37(\mathrm{~s}, 3 \mathrm{H}) .{ }^{13} \mathrm{C}$ NMR $\left(125 \mathrm{MHz}, \mathrm{CDCl}_{3}\right) \delta 176.0,144.7,139.8,136.0,134.4,133.0,130.0$ 127.5, 125.1, 121.4, 111.1, 94.1, 89.2, 21.8.

2.4 Procedure for the Preparation of Amine 7e 


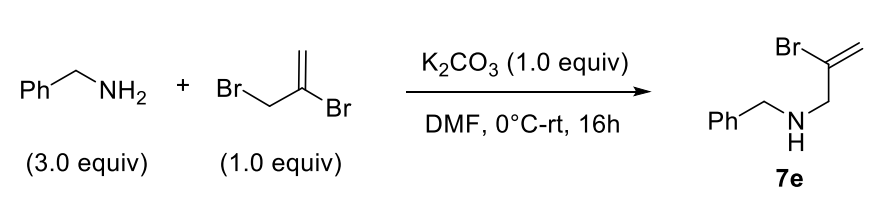

$\mathrm{N}$-benzyl-2-bromoprop-2-en-1-amine (7e). Adapting a literature procedure, ${ }^{5} \mathrm{~K}_{2} \mathrm{CO}_{3}$ ( $0.422 \mathrm{~g}, 3.055 \mathrm{mmol}, 1.0$ equiv) was added to a stirred solution of benzylamine $(1.0 \mathrm{~mL}, 9.164$ mmol, 3.0 equiv) and 2,3-bromopropene (0.373 mL, 80\% sln, 1.0 equiv) in DMF (45 mL) at 0 ${ }^{\circ} \mathrm{C}$. The reaction was warmed to room temperature and stirred for $16 \mathrm{~h}$ at which point $\mathrm{Et}_{2} \mathrm{O}(100$ $\mathrm{mL})$ and $\mathrm{H}_{2} \mathrm{O}(30 \mathrm{~mL})$ were added to the reaction mixture. The organic layer was separated, dried over $\mathrm{MgSO}_{4}$, concentrated in vacuo and subjected to flash column chromatography on silica gel (20\% EtOAc in hexanes) to furnish compound $7 \mathrm{e}$ in $62 \%$ yield $(0.431 \mathrm{~g}, 1.906 \mathrm{mmol})$ as a yellowish oil. The spectroscopic data matched the reported data for this compound. ${ }^{5} \mathrm{R}_{f}=$ $0.46\left(40 \%\right.$ EtOAc in hexanes). ${ }^{1} \mathrm{H}$ NMR $\left(500 \mathrm{MHz}, \mathrm{CDCl}_{3}\right) \delta 7.44-7.18(\mathrm{~m}, 5 \mathrm{H}), 5.82(\mathrm{~s}, 1 \mathrm{H})$, $5.61(\mathrm{~s}, 1 \mathrm{H}), 3.77(\mathrm{~s}, 2 \mathrm{H}), 3.49(\mathrm{~d}, J=1.3 \mathrm{~Hz}, 2 \mathrm{H}), 1.78(\mathrm{bs}, 1 \mathrm{H})$.

2.5 Procedure for the Preparation of Alkyne $\mathbf{8 f}$

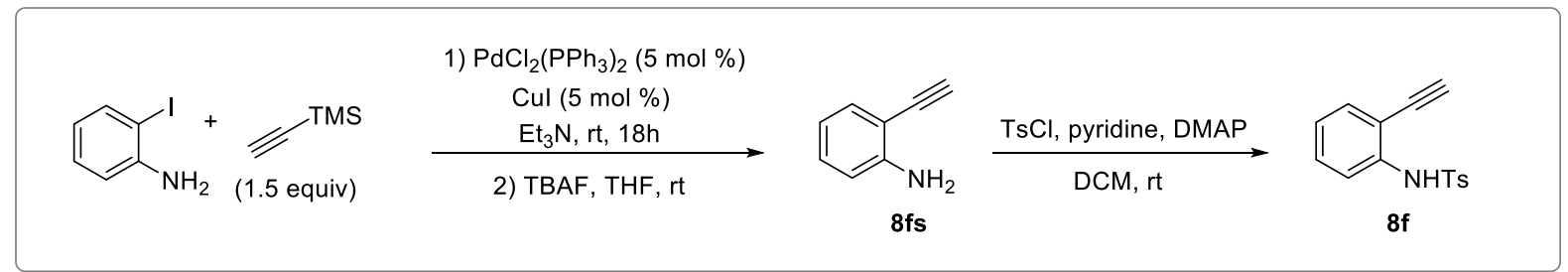

2-ethynylaniline (8fs). Adapting a literature procedure, ${ }^{6} \mathrm{CuI}(43.5 \mathrm{mg}, 0.23 \mathrm{mmol}, 5 \mathrm{~mol}$ \%), $\left(\mathrm{Ph}_{3} \mathrm{P}\right)_{2} \mathrm{PdCl}_{2}(160.2 \mathrm{mg}, 0.23 \mathrm{mmol}, 5 \mathrm{~mol} \%)$, and trimethylsilylacetylene $(0.97 \mathrm{~mL}, 6.85$ mmol, 1.5 equiv) were added to a stirred solution of 2-iodoaniline (1.00 g, $4.566 \mathrm{mmol}, 1.0$ equiv) in $\mathrm{Et}_{3} \mathrm{~N}$ (12.7 mL, $91.32 \mathrm{mmol}, 20$ equiv), at room temperature. The mixture was stirred for $18 \mathrm{~h}$, concentrated under reduced pressure, and submitted to flash colum chromatography on 
silica gel (20\% EtOAc in hexanes) to afford the coupling product $(0.416 \mathrm{~g}, 2.83 \mathrm{mmol})$ in $85 \%$ yield as a yellowish oil that was taken to the next step. $\mathrm{R}_{f}=0.60$ ( $20 \%$ EtOAc in hexanes). TBAF (1.0 M in THF, $1.90 \mathrm{~mL}, 1.90 \mathrm{mmol}, 1.5$ equiv) was added to a stirred solution of the silylacetylene obtained above in THF $(8.0 \mathrm{~mL})$, at room temperature. The reaction was stirred for 1h before the addition of EtOAc $(15 \mathrm{~mL})$ and $\mathrm{H}_{2} \mathrm{O}(5 \mathrm{~mL})$. The organic layer was separated, dried over $\mathrm{MgSO}_{4}$, concentrated, and subjected to flash column chromatography on silica gel (20-30\% EtOAc in hexanes) to furnish compound $\mathbf{8 f s}$ in $64 \%$ yield $(0.0952 \mathrm{~g}, 0.813 \mathrm{mmol})$ as a

yellowish oil. The spectroscopic data matched the reported data for this compound. ${ }^{6} \mathrm{R}_{f}=0.60$ (20\% EtOAc in hexanes). ${ }^{1} \mathrm{H}$ NMR $\left(500 \mathrm{MHz}, \mathrm{CDCl}_{3}\right) \delta 7.33(\mathrm{~d}, J=7.0 \mathrm{~Hz}, 1 \mathrm{H}), 7.16(\mathrm{t}, J=7.4$ $\mathrm{Hz}, 1 \mathrm{H}), 6.76-6.57(\mathrm{~m}, 2 \mathrm{H}), 4.25$ (bs, 2H), $3.40(\mathrm{~s}, 1 \mathrm{H})$.

N-(2-ethynylphenyl)-4-methylbenzenesulfonamide (8f). Pyridine $(0.16 \mathrm{~mL}, 1.94$ mmol, 5.0 equiv), catalytic DMAP ( 1 crystal), and $\mathrm{TsCl}(0.0813 \mathrm{~g}, 0.426 \mathrm{mmol}, 1.1$ equiv) was added to a stirred solution of $\mathbf{8} \mathbf{f s}$ in dichloromethane $(1.6 \mathrm{~mL})$, at room temperature. The mixture was stirred for $24 \mathrm{~h}$, diluted with dichloromethane $(4.0 \mathrm{~mL})$, and quenched with $\mathrm{NH}_{4} \mathrm{Cl}$ (saturated aqueous solution, $4.0 \mathrm{~mL}$ ). The organic layer was separated, dried over $\mathrm{MgSO}_{4}$, concentrated in vacuo, and subjected to flash column chromatography on silica gel $(2-10 \%$ EtOAc in hexanes gradient) to furnish compound $\mathbf{8 f}$ in $58 \%$ yield $(0.062 \mathrm{~g}, 0.229 \mathrm{mmol})$ as a white solid. The spectroscopic data matched the reported data for this compound. ${ }^{6} \mathrm{R}_{f}=0.43$ (20\% EtOAc in hexanes). ${ }^{1} \mathrm{H}$ NMR $\left(500 \mathrm{MHz}, \mathrm{CDCl}_{3}\right) \delta 7.70(\mathrm{~d}, J=8.4 \mathrm{~Hz}, 2 \mathrm{H}), 7.60(\mathrm{~d}, J=$ $8.4 \mathrm{~Hz}, 1 \mathrm{H}), 7.36-7.31(\mathrm{~d}, J=8.4 \mathrm{~Hz}, 1 \mathrm{H}), 7.31-7.24(\mathrm{~m}, 2 \mathrm{H}), 7.22(\mathrm{~d}, J=7.8 \mathrm{~Hz}, 2 \mathrm{H}), 7.01$ $(\operatorname{td}, J=7.8,1.2 \mathrm{~Hz}, 1 \mathrm{H}), 3.38(\mathrm{~s}, 1 \mathrm{H}), 2.36(\mathrm{~s}, 3 \mathrm{H}) \cdot{ }^{13} \mathrm{C}$ NMR $\left(125 \mathrm{MHz}, \mathrm{CDCl}_{3}\right) \delta$ 144.3, 138.6, 136.1, 132.7, 130.3, 129.8, 127.5, 124.3, 119.5, 112.8, 84.6, 78.8, 21.7. 


\section{Synthesis of 3-amino-1,4-diynes 9a-q}

3.1 General Procedure for the Preparation of 3-amino-1,4-diynes

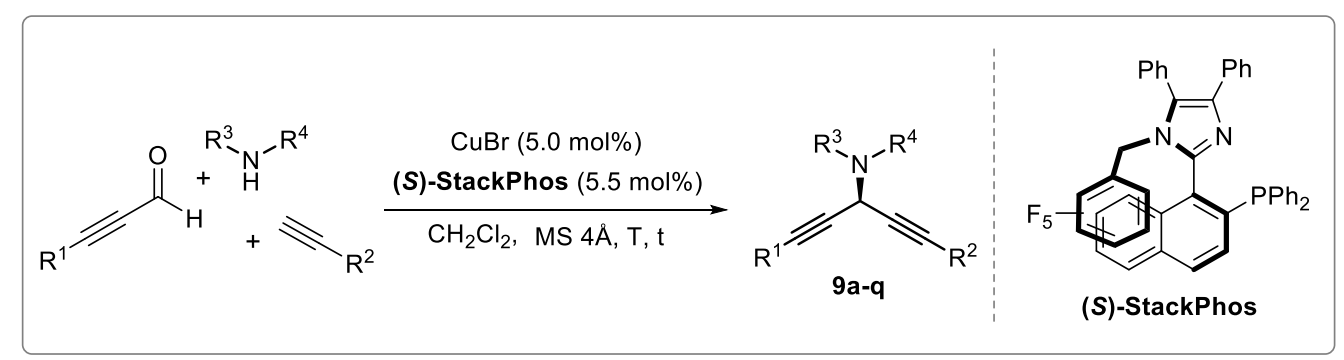

$\mathrm{CuBr}(0.5-2.0 \mathrm{mg}, 0.0035-0.0139 \mathrm{mmol}, 5 \mathrm{~mol} \%)$ and activated $4 \AA$ molecular sieves (50 $\mathrm{mg} / \mathrm{mg}$ of $\mathrm{CuBr}$ ) were added to an oven-dried test tube, which was then capped with a rubber septum. (S)-StackPhos (2.7-10.9 $\mathrm{mg}, 0.0038-0.0153 \mathrm{mmol}, 5.5 \mathrm{~mol} \%$ ) was added to the test tube, followed by anhydrous dichloromethane $(1.0 \mathrm{~mL} / \mathrm{mg}$ of $\mathrm{CuBr})$. The mixture was stirred for $20 \mathrm{~min}$ at room temperature and formation of a yellowish solution was observed. The alkyne (0.0697-0.2788 mmol, 1.0 equiv) and the aldehyde (0.0697-0.2788 mmol, 1.0 equiv) were then added to the tube and the mixture was cooled to $0^{\circ} \mathrm{C}$ for $5 \mathrm{~min}$, at which point the amine $(0.0697$ $0.2788 \mathrm{mmol}, 1.0$ equiv) and anhydrous dichloromethane $(0.4 \mathrm{~mL} / \mathrm{mg}$ of $\mathrm{CuBr})$ were added (final concentration of substrate in dichloromethane was $=0.1 \mathrm{M}$ ). The septum was then replaced with a plastic cap and during the course of the reaction. After the indicated time, flash column chromatography on silica gel (0-5\% EtOAc in hexanes, gradient) afforded the desired 3-amino1,4-diynes. For the preparation of racemic products, the same procedure was followed with racStackPhos. In the cases where TMS-acetylene and pentyne were employed, 1.5 equiv of the alkyne were added to the reactions due to their volatility. Any modification of this procedure, full characterization of compounds, as well as the determination of enantiomeric excesses can be found in the section 3.2. 
3.2 Characterization of 3-amino-1,4-diynes

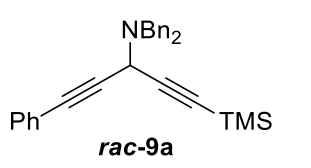

$N, N$-dibenzyl-1-phenyl-5-(trimethylsilyl)penta-1,4-diyn-3-amine (rac-9a).

$95 \%$ yield $(54.0 \mathrm{mg}, 0.132 \mathrm{mmol})$ as a yellowish oil. The reaction was carried out for $24 \mathrm{~h} . \mathrm{R}_{f}=$ $0.81\left(20 \%\right.$ EtOAc in hexanes, $\mathrm{UV} / \mathrm{KMnO}_{4}$ stain $) .{ }^{1} \mathrm{H} \mathrm{NMR}\left(500 \mathrm{MHz}, \mathrm{CDCl}_{3}\right) \delta 7.55(\mathrm{ddd}, J=$ 4.8, 3.4, 1.7 Hz, 2H), $7.52-7.47(\mathrm{~m}, 4 \mathrm{H}), 7.42-7.36(\mathrm{~m}, 7 \mathrm{H}), 7.35-7.29(\mathrm{~m}, 2 \mathrm{H}), 4.64(\mathrm{~s}, 1 \mathrm{H})$, $3.87(\mathrm{~d}, J=5.5 \mathrm{~Hz}, 2 \mathrm{H}), 3.82(\mathrm{~d}, J=5.5 \mathrm{~Hz}, 2 \mathrm{H}), 0.30(\mathrm{~s}, 9 \mathrm{H}) .{ }^{13} \mathrm{C} \mathrm{NMR}\left(125 \mathrm{MHz}, \mathrm{CDCl}_{3}\right) \delta$ 139.2, 132.1, 129.3, 128.5, 127.3, 123.0, 100.5, 89.2, 84.6, 84.3, 55.0, 45.5, 0.3. HRMS (ESITOF) $\mathrm{m} / \mathrm{z}:[\mathrm{M}+\mathrm{H}]^{+}$Calcd for $\mathrm{C}_{28} \mathrm{H}_{30} \mathrm{NSi}^{+}$408.2142; Found 408.2151.

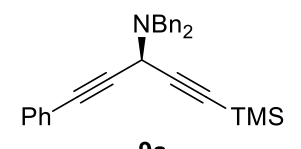

$9 \mathbf{a}$

\section{(R)-N,N-dibenzyl-1-phenyl-5-(trimethylsilyl)penta-1,4-diyn-3-amine (9a).} Compound 9a was prepared using procedure 3.1 with $(S)$-StackPhos in 73\% yield (95.2 $\mathrm{mg}, 0.233 \mathrm{mmol})$ as a white solid. The reaction was carried out for 24h. $[\alpha]^{24}=-69.65\left(c 1.0, \mathrm{CHCl}_{3}\right) . \mathrm{MP}=76^{\circ} \mathrm{C}$. Enantiomeric excess was determined by HPLC with a Chiralcel OD-H column (100:0 $n$-hexane:isopropanol, $0.2 \mathrm{~mL} / \mathrm{min}, 254 \mathrm{~nm})$; $\operatorname{minor} \mathrm{t}_{\mathrm{r}}=$ $30.2 \mathrm{~min}$; major $\mathrm{t}_{\mathrm{r}}=32.7 \mathrm{~min} ; 96 \%$ ee. The absolute configuration was determined in analogy to $9 b$.

Chromatograms of rac-9a and 9a:
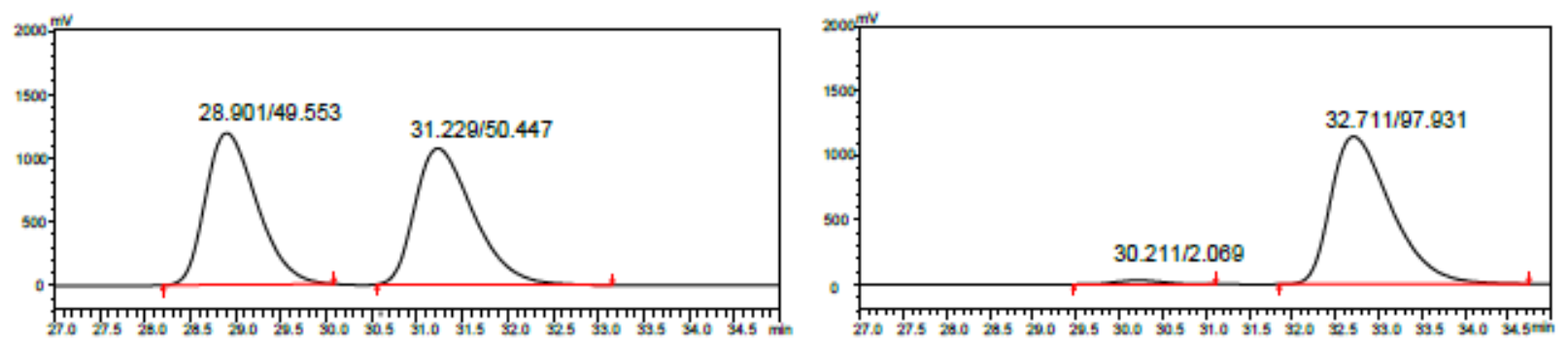


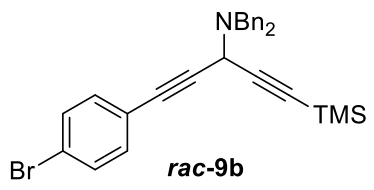

\section{$N, N$-dibenzyl-1-(4-bromophenyl)-5-(trimethylsilyl)penta-1,4-diyn-3-}

amine (rac-9b). Compound rac-9b was prepared using procedure 3.1 with rac-StackPhos in $83 \%$ yield $(30.8 \mathrm{mg}, 0.063 \mathrm{mmol})$ as a white solid. The reaction was carried out for $24 \mathrm{~h} . \mathrm{R}_{f}=0.79\left(20 \%\right.$ EtOAc in hexanes, UV/ $\mathrm{KMnO}_{4}$ stain). ${ }^{1} \mathrm{H}$ NMR (500 MHz, $\left.\mathrm{CDCl}_{3}\right) \delta 7.35-7.28(\mathrm{~m}, 6 \mathrm{H}), 7.24-7.19(\mathrm{~m}, 6 \mathrm{H}), 7.17-7.12(\mathrm{~m}$, 2H), $4.45(\mathrm{~s}, 1 \mathrm{H}), 3.67(\mathrm{~d}, J=13.5 \mathrm{~Hz}, 2 \mathrm{H}), 3.63(\mathrm{~d}, J=13.5 \mathrm{~Hz}, 2 \mathrm{H}), 0.12(\mathrm{~s}, 9 \mathrm{H}) .{ }^{13} \mathrm{C}$ NMR $\left(125 \mathrm{MHz}, \mathrm{CDCl}_{3}\right) \delta 139.1,133.6,131.7,129.2,128.5,127.4$ 122.8, 121.9, 100.0, 89.5, 86.0, 83.2, 55.1, 45.5, 0.3. HRMS (ESI-TOF) $\mathrm{m} / \mathrm{z}$ : $[\mathrm{M}+\mathrm{H}]^{+}$Calcd for $\mathrm{C}_{28} \mathrm{H}_{29} \mathrm{NSiBr}^{+}$486,1247; Found 486,1254 .

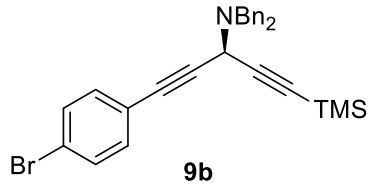

(R)-N,N-dibenzyl-1-(4-bromophenyl)-5-(trimethylsilyl)penta-1,4diyn-3-amine (9b). Compound 9b was prepared using procedure 3.1 with $(S)$-StackPhos in 79\% yield $(59.2 \mathrm{mg}, 0.122 \mathrm{mmol})$ as a white solid.

The reaction was carried out for $24 \mathrm{~h} .[\alpha]^{24} \mathrm{D}=-84.58\left(c 1.0, \mathrm{CHCl}_{3}\right) . \mathrm{MP}=109^{\circ} \mathrm{C}$. Enantiomeric excess was determined by HPLC with a Chiralcel OD-H column (100:0 $n$-hexane:isopropanol, $0.2 \mathrm{~mL} / \mathrm{min}, 254 \mathrm{~nm}$ ); minor $t_{\mathrm{r}}=47.2 \mathrm{~min}$; major $\mathrm{t}_{\mathrm{r}}=41.6 \mathrm{~min} ; 96 \%$ ee. The absolute configuration was determined by the optical rotation and retention time in the HPLC, which are the opposite if compared to those of compound ent-9b.

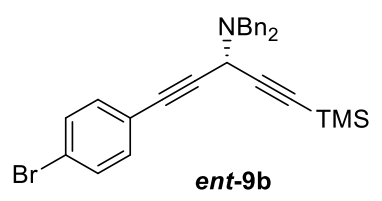

(S)-N,N-dibenzyl-1-(4-bromophenyl)-5-(trimethylsilyl)penta-1,4diyn-3-amine (ent-9b). Compound ent-9b was prepared using procedure 3.1 with $(R)$-StackPhos in $74 \%$ yield $(41.4 \mathrm{mg}, 0.085 \mathrm{mmol})$ as a white solid. The reaction was carried out for $24 \mathrm{~h} .[\alpha]^{24}{ }_{\mathrm{D}}=+64.25\left(c 0.5, \mathrm{CHCl}_{3}\right)$. Enantiomeric excess was determined by HPLC with a Chiralcel OD-H column (100:0 nhexane:isopropanol, $0.2 \mathrm{~mL} / \mathrm{min}, 254 \mathrm{~nm}$ ); minor $\mathrm{t}_{\mathrm{r}}=40.6 \mathrm{~min}$; major $\mathrm{t}_{\mathrm{r}}=45.1 \mathrm{~min} ; 96 \%$ ee. The 
absolute configuration was determined by single crystal X-Ray crystallography analysis with Flack parameter $=-0.013$ (See section 10 for more details).

Chromatograms of rac-9b and $\mathbf{9 b}$ :
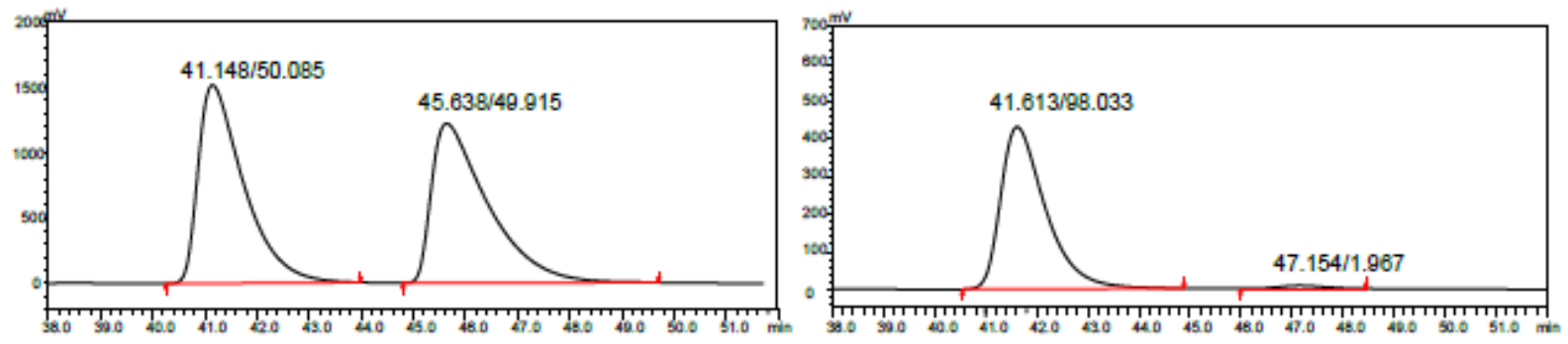

Chromatograms of rac-9b and ent-9b:
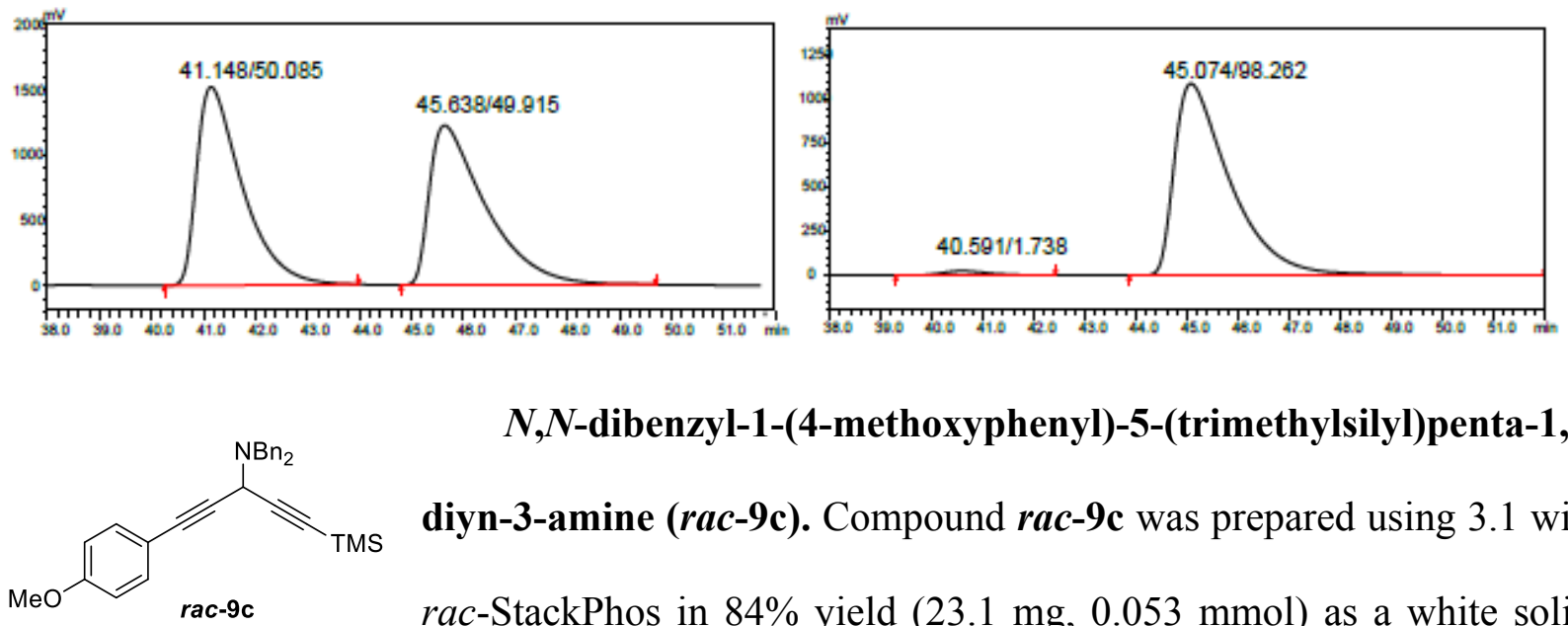

$N, N$-dibenzyl-1-(4-methoxyphenyl)-5-(trimethylsilyl)penta-1,4diyn-3-amine (rac-9c). Compound rac-9c was prepared using 3.1 with rac-StackPhos in $84 \%$ yield $(23.1 \mathrm{mg}, 0.053 \mathrm{mmol})$ as a white solid.

The reaction was carried out for $24 \mathrm{~h} . \mathrm{R}_{f}=0.62$ (20\% EtOAc in hexanes, UV/ $\mathrm{KMnO}_{4}$ stain). ${ }^{1} \mathrm{H}$ NMR (500 MHz, $\left.\mathrm{CDCl}_{3}\right) \delta 7.48-7.43(\mathrm{~m}, 6 \mathrm{H}), 7.39-7.34(\mathrm{~m}, 4 \mathrm{H}), 7.29(\mathrm{tt}, J=6.5,1.4 \mathrm{~Hz}$, 2H), $6.90-6.85(\mathrm{~m}, 2 \mathrm{H}), 4.59(\mathrm{~s}, 1 \mathrm{H}), 3.85(\mathrm{~s}, 3 \mathrm{H}), 3.84(\mathrm{~d}, J=13.4 \mathrm{~Hz}, 2 \mathrm{H}), 3.78(\mathrm{~d}, J=13.4$ $\mathrm{Hz}, 2 \mathrm{H}), 0.27$ (s, 9H). ${ }^{13} \mathrm{C}$ NMR (125 MHz, $\left.\mathrm{CDCl}_{3}\right) \delta 159.8,139.3,133.6,129.3,128.5,127.3$, $115.1,114.1,100.7,89.0,84.2,83.2,55.5,55.0,45.6,0.3$. HRMS (ESI-TOF) m/z: $[\mathrm{M}+\mathrm{H}]^{+}$ Calcd for $\mathrm{C}_{29} \mathrm{H}_{32} \mathrm{NOSi}^{+}$438.2248; Found 438.2256. 


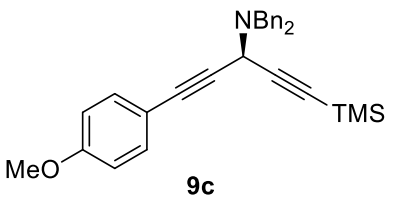

(R)-N,N-dibenzyl-1-(4-methoxyphenyl)-5-(trimethylsilyl)penta-1,4diyn-3-amine (9c). Compound 9c was prepared using procedure 3.1 with $(S)$-StackPhos in $77 \%$ yield $(32.7 \mathrm{mg}, 0.075 \mathrm{mmol})$ as a white solid. The reaction was carried out for $24 \mathrm{~h} .[\alpha]^{24}{ }_{\mathrm{D}}=-72.98\left(c 1.0, \mathrm{CHCl}_{3}\right) . \mathrm{MP}=85{ }^{\circ} \mathrm{C}$. Enantiomeric excess was determined by HPLC with a Chiralcel OD-H column (100:0 nhexane:isopropanol, $0.5 \mathrm{~mL} / \mathrm{min}, 254 \mathrm{~nm}$ ); minor $\mathrm{t}_{\mathrm{r}}=38.6 \mathrm{~min}$; major $\mathrm{t}_{\mathrm{r}}=42.6 \mathrm{~min} ; 94 \%$ ee. The absolute configuration was determined in analogy to $\mathbf{9 b}$.

Chromatograms of rac-9c and $9 \mathrm{c}$ :
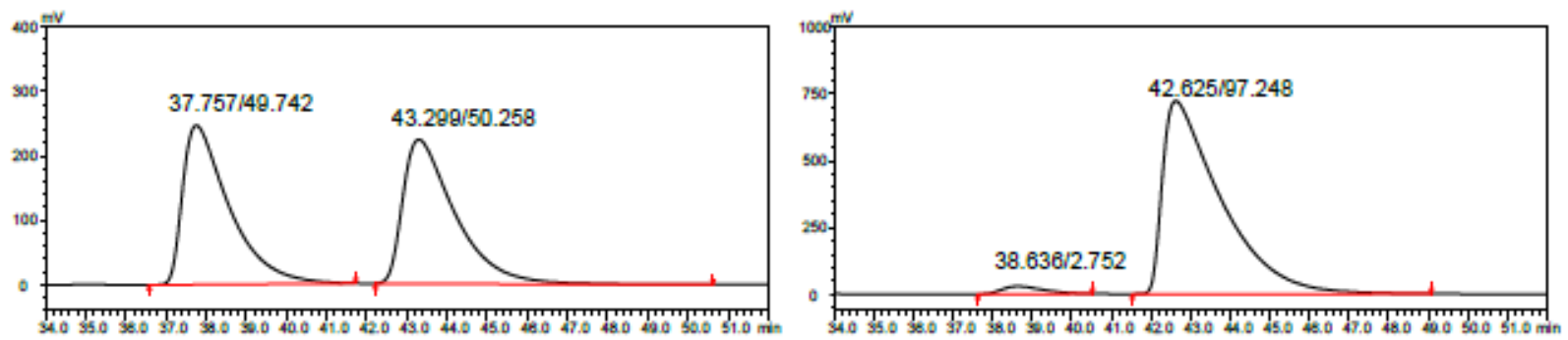

$N, N$-dibenzyl-1-(thiophen-2-yl)-5-(trimethylsilyl)penta-1,4-diyn-3-

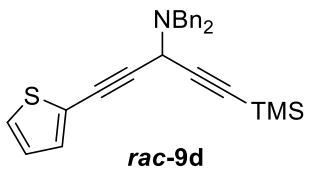

amine (rac-9d). Compound rac-9d was prepared using procedure 3.1 with rac-StackPhos in $44 \%$ yield $(20.1 \mathrm{mg}, 0.049 \mathrm{mmol})$ as a colorless oil. The

reaction was carried out for $24 \mathrm{~h} . \mathrm{R}_{f}=0.73\left(20 \%\right.$ EtOAc in hexanes, UV/ $\mathrm{KMnO}_{4}$ stain). ${ }^{1} \mathrm{H}$ $\operatorname{NMR}\left(500 \mathrm{MHz}, \mathrm{CDCl}_{3}\right) \delta 7.49-7.44(\mathrm{~m}, 4 \mathrm{H}), 7.38(\mathrm{t}, J=7.5 \mathrm{~Hz}, 4 \mathrm{H}), 7.34-7.26(\mathrm{~m}, 4 \mathrm{H})$, $7.02(\mathrm{dd}, J=5.1,3.6 \mathrm{~Hz}, 1 \mathrm{H}), 4.64(\mathrm{~s}, 1 \mathrm{H}), 3.81(\mathrm{~s}, 4 \mathrm{H}), 0.28(\mathrm{~s}, 9 \mathrm{H}) .{ }^{13} \mathrm{C}$ NMR $(125 \mathrm{MHz}$, $\left.\mathrm{CDCl}_{3}\right) \delta 139.1,132.6,129.3,128.5,127.4,127.3,127.1,122.9,100.2,89.5,88.6,77.6,55.1$, 45.8, 0.3. HRMS (DART-TOF) m/z: $[\mathrm{M}+\mathrm{H}]^{+}$Calcd for $\mathrm{C}_{26} \mathrm{H}_{28} \mathrm{NSSi}^{+}$414.1706; Found 414,1714. 


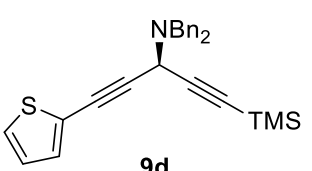

$9 d$

reaction was carried out for $24 \mathrm{~h} .[\alpha]_{\mathrm{D}}^{24}=-59.80\left(c 1.0, \mathrm{CHCl}_{3}\right)$. Enantiomeric excess was determined to be $96 \%$ ee after desilylation (see procedure for 9d' below). The absolute configuration was determined in analogy to $9 \mathbf{b}$.

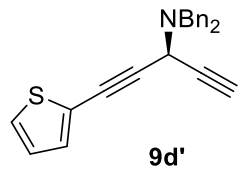

(S)- $N, N$-dibenzyl-1-(thiophen-2-yl)penta-1,4-diyn-3-amine (9d'). KF $\quad(9.5$ $\mathrm{mg}, 0.25 \mathrm{mmol}, 10.0$ equiv) was added to a stirred solution of $\mathbf{9 d}(10.3 \mathrm{mg}$, $0.025 \mathrm{mmol}, 1.0$ equiv) in $\mathrm{MeCN}(1.0 \mathrm{~mL})$ and $\mathrm{H}_{2} \mathrm{O}(0.25 \mathrm{~mL})$, at room temperature and resulting solution was stirred for $16 \mathrm{~h}$. The mixture was then concentrated under reduced pressure and EtOAc $(2.0 \mathrm{~mL})$ was added. The organic layer was washed with water $(1.0$ $\mathrm{mL}$ ), separated, dried over $\mathrm{MgSO}_{4}$, concentrated, and subjected to flash column chromatography (0-5\% EtOAc in hexanes) to deliver compound 9d' in $75 \%$ yield $(6.2 \mathrm{mg}, 0.018 \mathrm{mmol})$ as a colorless oil. $\mathrm{R}_{f}=0.58$ (20\% EtOAc in hexanes, UV/ $\mathrm{KMnO}_{4}$ stain). ${ }^{1} \mathrm{H}$ NMR (500 MHz, $\left.\mathrm{CDCl}_{3}\right) \delta 7.48-7.44(\mathrm{~m}, 4 \mathrm{H}), 7.39-7.34(\mathrm{~m}, 4 \mathrm{H}), 7.32-7.26(\mathrm{~m}, 4 \mathrm{H}), 7.01(\mathrm{dd}, J=5.1,3.7$ $\mathrm{Hz}, 1 \mathrm{H}), 4.64(\mathrm{~d}, J=2.4 \mathrm{~Hz}, 1 \mathrm{H}), 3.82(\mathrm{~s}, 4 \mathrm{H}), 2.48(\mathrm{~d}, J=2.4 \mathrm{~Hz}, 1 \mathrm{H}) .{ }^{13} \mathrm{C}$ NMR $(125 \mathrm{MHz}$ $\left.\mathrm{CDCl}_{3}\right) \delta 138.9,132.7,129.2,128.6,127.5,127.1,122.7,88.1,78.7,77.8,72.6,55.0,44.9$. HRMS (ESI-TOF) m/z: $[\mathrm{M}+\mathrm{H}]^{+}$Calcd for $\mathrm{C}_{23} \mathrm{H}_{19} \mathrm{NS}^{+}$342.1311; Found 342.1319. $[\alpha]^{24}{ }_{\mathrm{D}}=-$ $124.31\left(c\right.$ 0.5, $\left.\mathrm{CHCl}_{3}\right)$. Enantiomeric excess was determined by HPLC with a Chiralcel OD-H column (99.9:0.1 $n$-hexane:isopropanol, $0.5 \mathrm{~mL} / \mathrm{min}, 215 \mathrm{~nm}$ ); minor $t_{\mathrm{r}}=26.7 \mathrm{~min}$; major $\mathrm{t}_{\mathrm{r}}=$ $31.7 \mathrm{~min} ; 96 \%$ ee. The absolute configuration was determined in analogy to $\mathbf{9 d}$. 
Chromatograms of rac-9d' and 9d':
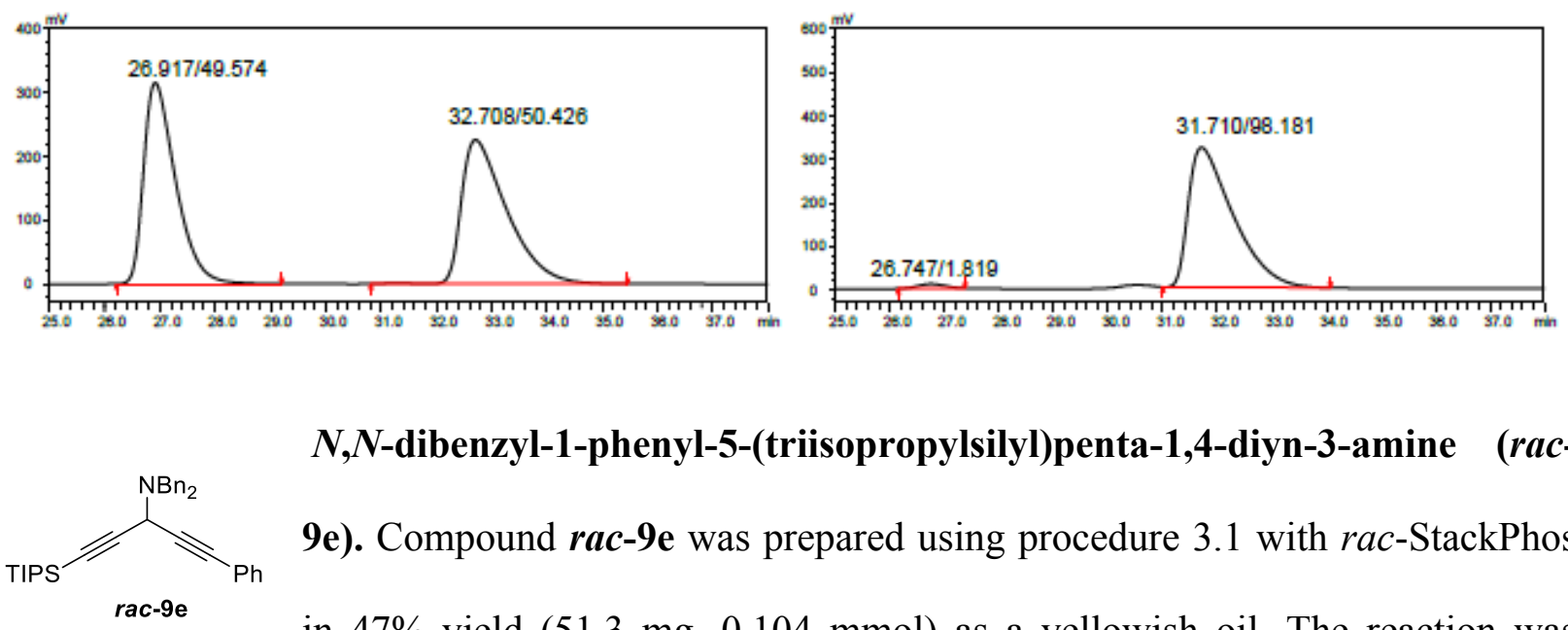

$N, N$-dibenzyl-1-phenyl-5-(triisopropylsilyl)penta-1,4-diyn-3-amine $\quad$ (rac-

9e). Compound rac-9e was prepared using procedure 3.1 with $r a c$-StackPhos in $47 \%$ yield $(51.3 \mathrm{mg}, 0.104 \mathrm{mmol})$ as a yellowish oil. The reaction was carried out for $24 \mathrm{~h} . \mathrm{R}_{f}=0.57$ (10\% EtOAc in hexanes, UV/ $\mathrm{KMnO}_{4}$ stain). ${ }^{1} \mathrm{H}$ NMR (500 MHz, $\left.\mathrm{CDCl}_{3}\right) \delta 7.51-7.45(\mathrm{~m}, 7 \mathrm{H}), 7.39-7.33(\mathrm{~m}, 6 \mathrm{H}), 7.32-7.27(\mathrm{~m}, 2 \mathrm{H}), 4.63(\mathrm{~s}, 1 \mathrm{H}), 3.92(\mathrm{~d}, J$ $=13.4 \mathrm{~Hz}, 2 \mathrm{H}), 3.78(\mathrm{~d}, J=13.4 \mathrm{~Hz}, 2 \mathrm{H}), 1.18(\mathrm{~s}, 9 \mathrm{H}) .{ }^{13} \mathrm{C} \mathrm{NMR}\left(125 \mathrm{MHz}, \mathrm{CDCl}_{3}\right) \delta 139.3$, $132.1,129.3,128.5,128.4,127.3,123.2,102.2,85.5,85.3,83.9,55.1,45.6,18.9,11.5$. HRMS (ESI-TOF) m/z: [M+H] ${ }^{+}$Calcd for $\mathrm{C}_{34} \mathrm{H}_{42} \mathrm{NSi}^{+}$492.3081; Found 492.3087.

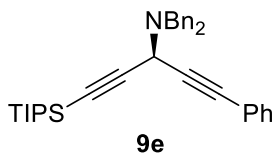

(S)- $N, N$-dibenzyl-1-phenyl-5-(triisopropylsilyl)penta-1,4-diyn-3-amine

out for $24 \mathrm{~h} .[\alpha]^{24}{ }_{\mathrm{D}}=+29.69\left(c 1.0, \mathrm{CHCl}_{3}\right)$. Enantiomeric excess was determined by HPLC with a Chiralcel OD-H column (100:0 $n$-hexane:isopropanol, $0.25 \mathrm{~mL} / \mathrm{min}, 254 \mathrm{~nm})$; minor $\mathrm{t}_{\mathrm{r}}=23.1$ $\min$; major $\mathrm{t}_{\mathrm{r}}=22.1 \mathrm{~min} ; 96 \%$ ee. The absolute configuration was determined in analogy to $\mathbf{9 b}$. 
Chromatograms of rac-9e and $9 \mathrm{e}$ :
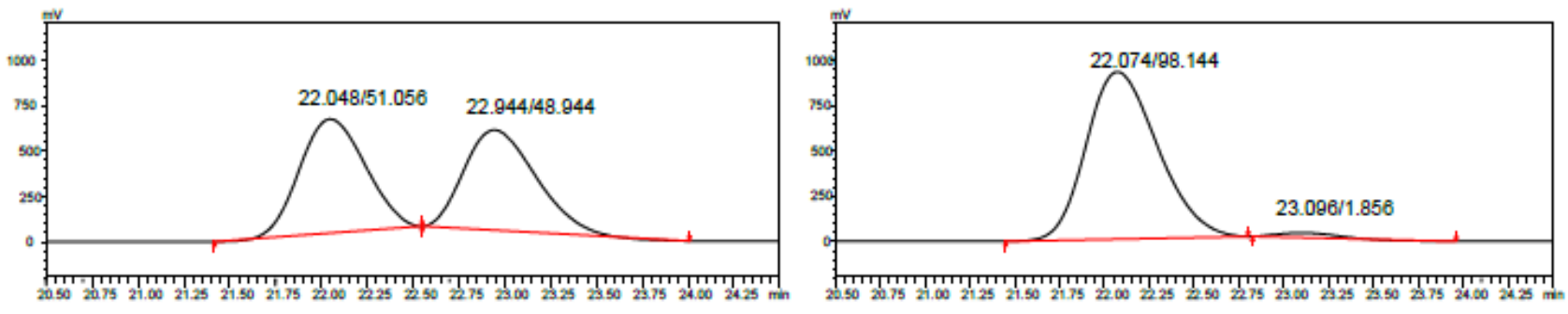

$N, N$-dibenzyl-1-(triisopropylsilyl)-5-(trimethylsilyl)penta-1,4-diyn-3-

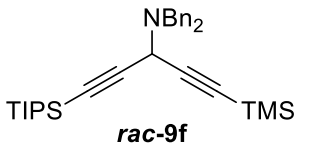

amine (rac-9f). Compound rac-9f was prepared using procedure 3.1 with rac-StackPhos in $82 \%$ yield $(140.1 \mathrm{mg}, 0.287 \mathrm{mmol})$ as a yellowish oil. The reaction was carried out for $6 \mathrm{~h} . \mathrm{R}_{f}=0.83\left(20 \%\right.$ EtOAc in hexanes, $\mathrm{UV} / \mathrm{KMnO}_{4}$ stain). ${ }^{1} \mathrm{H} \mathrm{NMR}$ $\left(500 \mathrm{MHz}, \mathrm{CDCl}_{3}\right) \delta 7.42(\mathrm{~d}, J=7.5 \mathrm{~Hz}, 4 \mathrm{H}), 7.34(\mathrm{t}, J=7.5 \mathrm{~Hz}, 4 \mathrm{H}), 7.31-7.23(\mathrm{~m}, 2 \mathrm{H}), 4.38$ (s, 1H), $3.82(\mathrm{~d}, J=13.4 \mathrm{~Hz}, 2 \mathrm{H}), 3.69(\mathrm{~d}, J=13.4 \mathrm{~Hz}, 2 \mathrm{H}), 1.15(\mathrm{~s}, 21 \mathrm{H}), 0.22(\mathrm{~s}, 9 \mathrm{H}) .{ }^{13} \mathrm{C}$ NMR $\left(125 \mathrm{MHz}\right.$, acetone- $\left.d_{6}\right) \delta 139.9,130.0,129.5,128.4,103.5,101.8,89.7,86.1,55.6,46.4$, 19.3, 12.3, 0.3. HRMS (ESI-TOF) $\mathrm{m} / \mathrm{z}:[\mathrm{M}+\mathrm{H}]^{+}$Calcd for $\mathrm{C}_{31} \mathrm{H}_{46} \mathrm{NSi}_{2}{ }^{+}$488.3163; Found 488.3174 .

\section{(S)-N,N-dibenzyl-1-(triisopropylsilyl)-5-(trimethylsilyl)penta-1,4-diyn-}

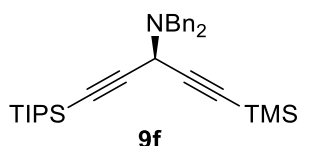

3-amine (9f). Compound 9f was prepared using procedure 3.1 with $(S)$ StackPhos in $72 \%$ yield $(34.2 \mathrm{mg}, 0.070 \mathrm{mmol})$ as a yellowish oil. The reaction was carried out for $6 \mathrm{~h}$. Enantiomeric excess was determined to be $96 \%$ ee after TMSdeprotection (see synthesis of $\mathbf{1 3}$ in section 4.3). The absolute configuration was determined by analogy to $\mathbf{9 b}$.

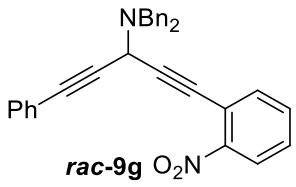

$N, N$-dibenzyl-1-(2-nitrophenyl)-5-phenylpenta-1,4-diyn-3-amine $\quad$ (rac-

9g). Compound rac-9g was prepared using procedure 3.1 with rac-StackPhos 
in $77 \%$ yield ( $34.6 \mathrm{mg}, 0.101 \mathrm{mmol}$ ) as a yellowish oil. The reaction was carried out for $3 \mathrm{~h} . \mathrm{R}_{f}=$ $0.43\left(20 \%\right.$ EtOAc in hexanes, UV/ $\mathrm{KMnO}_{4}$ stain). ${ }^{1} \mathrm{H}$ NMR $\left(500 \mathrm{MHz}, \mathrm{CDCl}_{3}\right) \delta 8.11$ (dd, $J=$ 8.3, $1.3 \mathrm{~Hz}, 1 \mathrm{H}), 7.73(\mathrm{dd}, J=7.8,1.5 \mathrm{~Hz}, 1 \mathrm{H}), 7.62(\mathrm{td}, J=7.6,1.3 \mathrm{~Hz}, 1 \mathrm{H}), 7.57-7.48(\mathrm{~m}$, 7H), $7.41-7.34(\mathrm{~m}, 7 \mathrm{H}), 7.33-7.29(\mathrm{~m}, 2 \mathrm{H}), 4.92(\mathrm{~s}, 1 \mathrm{H}), 3.99(\mathrm{~d}, J=13.5 \mathrm{~Hz}, 2 \mathrm{H}), 3.92(\mathrm{~d}, J$ $=13.5 \mathrm{~Hz}, 2 \mathrm{H}) .{ }^{13} \mathrm{C} \mathrm{NMR}\left(125 \mathrm{MHz}, \mathrm{CDCl}_{3}\right) \delta 150.1,139.1,135.4,133.0,132.2,129.3,129.0$, $128.7,128.6,128.5,127.4,124.8,122.8,118.4,93.0,84.7,84.2,79.8,55.2,45.7$. HRMS (ESITOF) m/z: $[\mathrm{M}+\mathrm{H}]^{+}$Calcd for $\mathrm{C}_{31} \mathrm{H}_{25} \mathrm{~N}_{2} \mathrm{O}_{2}{ }^{+}$457.1911; Found 457.1922.

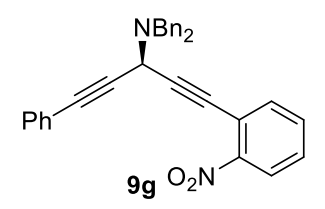

(R)- $\quad N, N$-dibenzyl-1-(2-nitrophenyl)-5-phenylpenta-1,4-diyn-3-amine

$(9 \mathrm{~g})$. Compound $9 \mathrm{~g}$ was prepared using procedure 3.1 with $(S)$-StackPhos in $86 \%$ yield $(38.5 \mathrm{mg}, 0.084 \mathrm{mmol})$ as a yellowish oil. The reaction was carried out for 3h. $[\alpha]^{24}=-4.00\left(c 1.0, \mathrm{CHCl}_{3}\right)$. Enantiomeric excess was determined by HPLC with a Chiralcel OD-H column (99.9:0.1 $n$-hexane:isopropanol, $0.5 \mathrm{~mL} / \mathrm{min}, 215 \mathrm{~nm})$; $\operatorname{minor} \mathrm{t}_{\mathrm{r}}=$ $75.1 \mathrm{~min}$; major $\mathrm{t}_{\mathrm{r}}=80.9 \mathrm{~min} ; 95 \%$ ee. The absolute configuration was determined in analogy to compound 9b.

Chromatograms of $\mathbf{r a c}-\mathbf{9 g}$ and $\mathbf{9 g}$ :
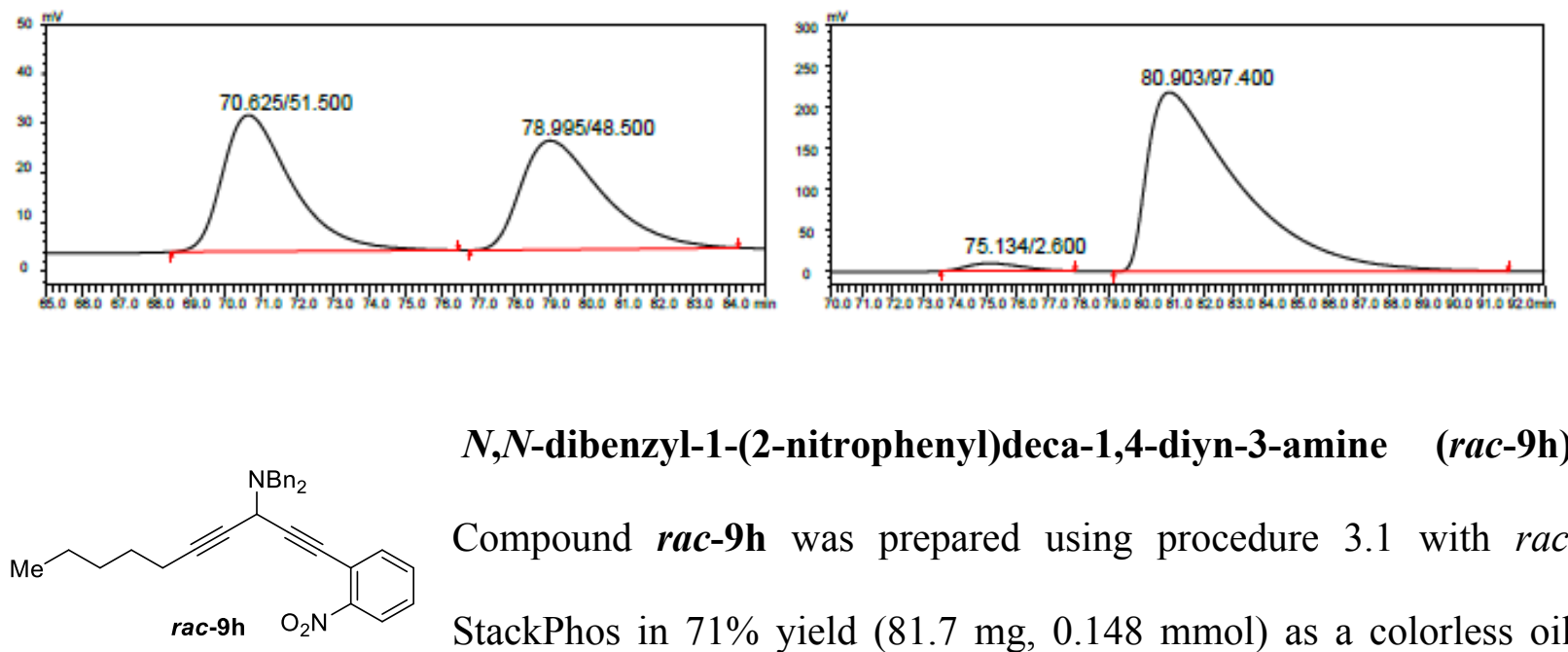

$N, N$-dibenzyl-1-(2-nitrophenyl)deca-1,4-diyn-3-amine $\quad$ (rac-9h).

Compound rac-9h was prepared using procedure 3.1 with racStackPhos in $71 \%$ yield $(81.7 \mathrm{mg}, 0.148 \mathrm{mmol})$ as a colorless oil. 
The reaction was carried out for $3 \mathrm{~h} . \mathrm{R}_{f}=0.59$ (20\% EtOAc in hexanes, UV/ $\mathrm{KMnO}_{4}$ stain). ${ }^{1} \mathrm{H}$ NMR (500 MHz, $\left.\mathrm{CDCl}_{3}\right) \delta 8.07(\mathrm{~d}, J=7.9 \mathrm{~Hz}, 1 \mathrm{H}), 7.68(\mathrm{~d}, J=7.7 \mathrm{~Hz}, 1 \mathrm{H}), 7.61-7.55(\mathrm{~m}$, 1H), $7.48(\mathrm{~d}, J=7.6 \mathrm{~Hz}, 5 \mathrm{H}), 7.35$ (t, $J=7.5 \mathrm{~Hz}, 4 \mathrm{H}), 7.27(\mathrm{t}, J=7.3 \mathrm{~Hz}, 2 \mathrm{H}), 4.65$ (s, 1H), 3.90 $(\mathrm{d}, J=13.5 \mathrm{~Hz}, 2 \mathrm{H}), 3.81(\mathrm{~d}, J=13.5 \mathrm{~Hz}, 2 \mathrm{H}), 2.29(\mathrm{td}, J=7.2,2.2 \mathrm{~Hz}, 2 \mathrm{H}), 1.59(\mathrm{p}, J=7.2 \mathrm{~Hz}$, 2H), $1.49-1.29(\mathrm{~m}, 4 \mathrm{H}), 0.93(\mathrm{t}, J=7.2 \mathrm{~Hz}, 3 \mathrm{H}) .{ }^{13} \mathrm{C} \mathrm{NMR}\left(125 \mathrm{MHz}, \mathrm{CDCl}_{3}\right) \delta 150.1,139.3$, $135.4,132.9,129.3,128.9,128.5,127.3,124.8,118.5,93.9,85.5,79.3,74.8,54.9,45.2,31.3$, 28.6, 22.4, 19.0, 14.2. HRMS (ESI-TOF) m/z: $[\mathrm{M}+\mathrm{H}]^{+}$Calcd for $\mathrm{C}_{30} \mathrm{H}_{31} \mathrm{~N}_{2} \mathrm{O}_{2}{ }^{+}$451.2380; Found 451.2388.

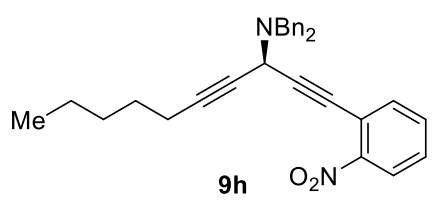

$(R)-N, N$-dibenzyl-1-(2-nitrophenyl)deca-1,4-diyn-3-amine $\quad(9 \mathrm{~h})$. Compound 9h was prepared using procedure 3.1 with $(S)$-StackPhos in $64 \%$ yield $(29.3 \mathrm{mg}, 0.053 \mathrm{mmol})$ as a colorless oil. The reaction was carried out for $6 \mathrm{~h} .[\alpha]_{\mathrm{D}}^{24}=+57.80\left(c\right.$ 1.0, $\left.\mathrm{CHCl}_{3}\right)$. Enantiomeric excess was determined by HPLC with a Chiralcel OD-H column (100:0 n-hexane:isopropanol, 0.5 mL/min, $254 \mathrm{~nm}$ ); minor $\mathrm{t}_{\mathrm{r}}=109.9 \mathrm{~min}$; major $\mathrm{t}_{\mathrm{r}}=114.2 \mathrm{~min} ; 96 \%$ ee. The absolute configuration was determined in analogy to $\mathbf{9 b}$.

Chromatograms of rac-9h and $\mathbf{9 h}$ :
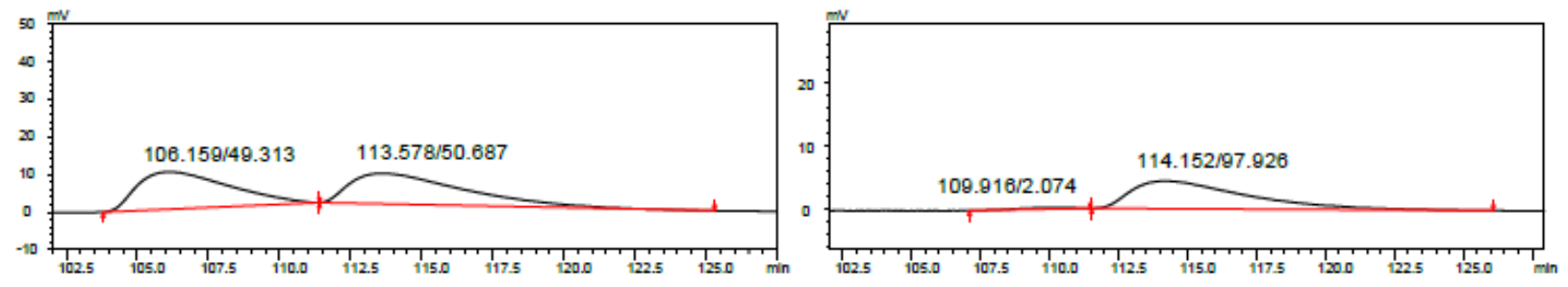

$N, N$-dibenzyl-1-phenylocta-1,4-diyn-3-amine (rac-9i). Compound rac-9i

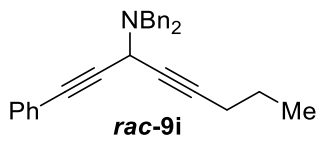

was prepared using procedure 3.1 with $\mathrm{rac}$-StackPhos in $72 \%$ yield $(36.2$ $\mathrm{mg}, 0.096 \mathrm{mmol}$ ) as a colorless oil. The reaction was carried out for $24 \mathrm{~h} . \mathrm{R}_{f}$ 
$=0.73\left(20 \%\right.$ EtOAc in hexanes, $\mathrm{UV} / \mathrm{KMnO}_{4}$ stain $) .{ }^{1} \mathrm{H} \mathrm{NMR}\left(500 \mathrm{MHz}, \mathrm{CDCl}_{3}\right) \delta 7.55-7.52$ (m, 2H), $7.51-7.47(\mathrm{~m}, 4 \mathrm{H}), 7.41-7.33(\mathrm{~m}, 7 \mathrm{H}), 7.33-7.28(\mathrm{~m}, 2 \mathrm{H}), 4.62(\mathrm{t}, J=2.2 \mathrm{~Hz}, 1 \mathrm{H})$, $3.86(\mathrm{~d}, J=13.5 \mathrm{~Hz}, 2 \mathrm{H}), 3.83(\mathrm{~d}, J=13.5 \mathrm{~Hz}, 2 \mathrm{H}), 2.31(\mathrm{td}, J=7.0,2.2 \mathrm{~Hz}, 2 \mathrm{H}), 1.65(\mathrm{~h}, J=$ $7.3 \mathrm{~Hz}, 2 \mathrm{H}), 1.09(\mathrm{t}, J=7.4 \mathrm{~Hz}, 3 \mathrm{H}) .{ }^{13} \mathrm{C}$ NMR $\left(125 \mathrm{MHz}, \mathrm{CDCl}_{3}\right) \delta 139.4,132.1,129.2,128.5$, 128.4, 127.3, 123.1, 85.5, 84.9, 83.9, 75.4, 55.0, 44.9, 22.5, 21.0, 13.8. HRMS (ESI-TOF) m/z: $[\mathrm{M}+\mathrm{H}]^{+}$Calcd for $\mathrm{C}_{28} \mathrm{H}_{28} \mathrm{~N}^{+}$378.2216; Found 378.2225.

(S)- $N, N$-dibenzyl-1-phenylocta-1,4-diyn-3-amine (9i). Compound 9i $\sim_{M e}^{\mathrm{NBn}_{2}}$ was prepared using procedure 3.1 with $(S)$-StackPhos in $84 \%$ yield $(27.8$ $\mathrm{mg}, 0.074 \mathrm{mmol}$ ) as a colorless oil. The reaction was carried out for $24 \mathrm{~h}$ and $9 \mathbf{i}$ was isolated in $86 \%$ ee. $[\alpha]^{24}=-69.37\left(c 1.0, \mathrm{CHCl}_{3}, 86 \%\right.$ ee sample). The preparation of compound $9 \mathbf{i}$ was also carried out for $24 \mathrm{~h}$, at $-25^{\circ} \mathrm{C}$, generating $9 \mathrm{i}$ in $65 \%$ yield $(21.3 \mathrm{mg}, 0.057$ mmol) and $88 \%$ ee. Enantiomeric excess was determined by HPLC with a Chiralcel OD-H column (100:0 $n$-hexane:isopropanol, $1.0 \mathrm{~mL} / \mathrm{min}, 254 \mathrm{~nm})$; minor $\mathrm{t}_{\mathrm{r}}=11.0 \mathrm{~min}$; major $\mathrm{t}_{\mathrm{r}}=15.4$ min. The absolute configuration was determined in analogy to compound $\mathbf{9 b}$.

Chromatograms of rac-9i and 9i:
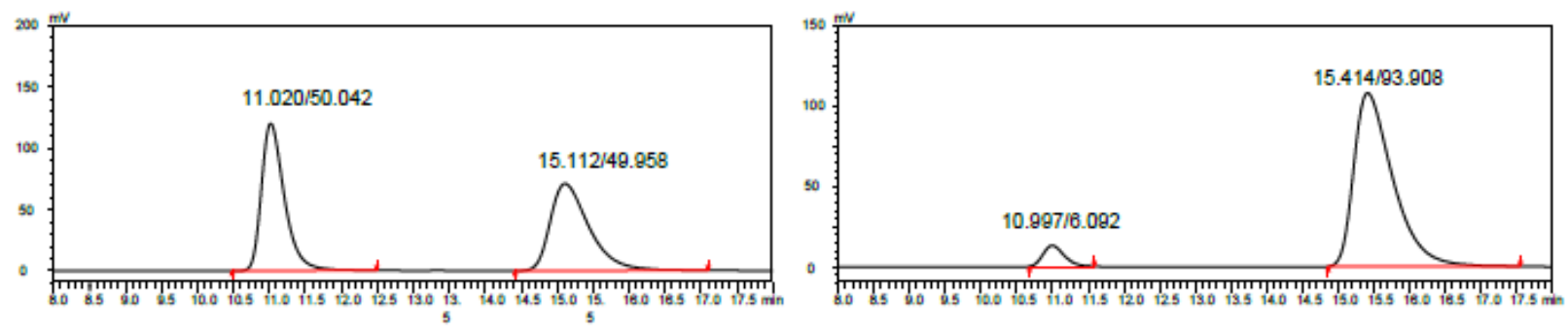

$N, N$-dibenzyltrideca-4,7-diyn-6-amine (rac-9j). Compound rac-

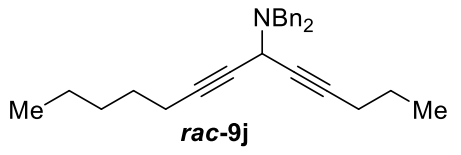

9j was prepared using procedure 3.1 with $\mathrm{rac}$-StackPhos in $93 \%$ yield $(97.4 \mathrm{mg}, 0.277 \mathrm{mmol})$ as a colorless oil. The reaction was 
carried out for $24 \mathrm{~h} . \mathrm{R}_{f}=0.82\left(20 \%\right.$ EtOAc in hexanes, UV/ $\mathrm{KMnO}_{4}$ stain). ${ }^{1} \mathrm{H} \mathrm{NMR}(500 \mathrm{MHz}$, $\left.\mathrm{CDCl}_{3}\right) \delta 7.42(\mathrm{~d}, J=6.6 \mathrm{~Hz}, 4 \mathrm{H}), 7.32(\mathrm{t}, J=7.1 \mathrm{~Hz}, 4 \mathrm{H}), 7.30-7.15(\mathrm{~m}, 2 \mathrm{H}), 4.34(\mathrm{~s}, 1 \mathrm{H})$, $3.72(\mathrm{~s}, 4 \mathrm{H}), 2.32-2.16(\mathrm{~m}, 4 \mathrm{H}), 1.69-1.50(\mathrm{~m}, 4 \mathrm{H}), 1.49-1.30(\mathrm{~m}, 4 \mathrm{H}), 1.04(\mathrm{td}, J=7.4,1.5$ $\mathrm{Hz}, 3 \mathrm{H}), 0.92(\mathrm{td}, J=7.3,1.4 \mathrm{~Hz}, 3 \mathrm{H}) .{ }^{13} \mathrm{C} \mathrm{NMR}\left(125 \mathrm{MHz}, \mathrm{CDCl}_{3}\right) \delta 139.6,129.2,128.4$, $127.2,84.4,84.3,76.1,76.0,54.8,44.4,31.3,28.7,22.5,21.0,19.0,14.2,13.8$. HRMS (ESITOF) m/z: $[\mathrm{M}+\mathrm{H}]^{+}$Calcd for $\mathrm{C}_{27} \mathrm{H}_{34} \mathrm{~N}^{+} 372.2686$; Found 372.2695 .

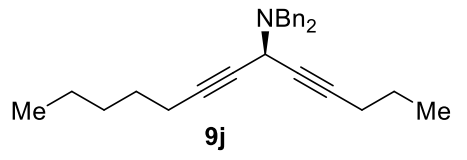

(S)-N,N-dibenzyltrideca-4,7-diyn-6-amine (9j). Compound 9j was prepared using procedure 3.1 with $(S)$-StackPhos in $86 \%$ yield (17.9 $\mathrm{mg}, 0.051 \mathrm{mmol})$ as a colorless oil. The reaction was carried out for $24 \mathrm{~h} .[\alpha]^{24}=-8.70\left(c 1.0, \mathrm{CHCl}_{3}\right)$. Enantiomeric excess was determined to be $82 \%$ by HPLC of the corresponding Mosher's amide 18 (91: $9 \mathrm{dr}$, see synthesis and chromatograms in section 4.4). The absolute configuration was determined in analogy to $\mathbf{9 b}$.

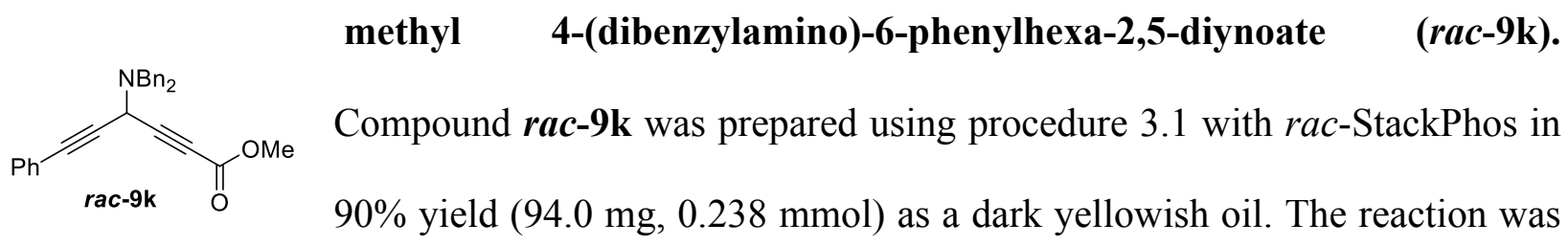
carried out for $3 \mathrm{~h} . \mathrm{R}_{f}=0.50$ (20\% EtOAc in hexanes, UV/ $\mathrm{KMnO}_{4}$ stain). ${ }^{1} \mathrm{H}$ NMR (500 MHz, $\left.\mathrm{CDCl}_{3}\right) \delta 7.53-7.50(\mathrm{~m}, 2 \mathrm{H}), 7.47(\mathrm{~d}, J=7.0 \mathrm{~Hz}, 4 \mathrm{H}), 7.40-7.36(\mathrm{~m}, 7 \mathrm{H}), 7.33-7.28(\mathrm{~m}$, 2H), $4.76(\mathrm{~s}, 1 \mathrm{H}), 3.90(\mathrm{~d}, J=13.5 \mathrm{~Hz}, 2 \mathrm{H}), 3.85(\mathrm{~s}, 3 \mathrm{H}), 3.79(\mathrm{~d}, J=13.5 \mathrm{~Hz}, 2 \mathrm{H}) .{ }^{13} \mathrm{C} \mathrm{NMR}$ $\left(125 \mathrm{MHz}, \mathrm{CDCl}_{3}\right) \delta 153.9,138.5,132.2,129.2,128.9,128.7,128.5,127.6,122.3,85.6,82.9$, 82.3,75.8, 55.2, 53.1, 44.9. HRMS (DART-TOF) m/z: $[\mathrm{M}+\mathrm{H}]^{+}$Calcd for $\mathrm{C}_{27} \mathrm{H}_{24} \mathrm{NO}_{2}{ }^{+}$394.1802; Found 394.1819. 


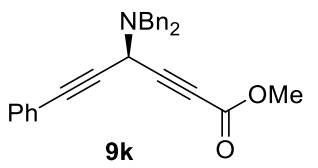

methyl (R)-4-(dibenzylamino)-6-phenylhexa-2,5-diynoate

(9k).

Compound 9k was prepared using procedure 3.1 with $(S)$-StackPhos in $96 \%$ yield $(50.5 \mathrm{mg}, 0.128 \mathrm{mmol})$ as a dark yellowish oil. The reaction was carried out for $1 \mathrm{~h}$ and $9 \mathbf{k}$ was isolated in $82 \%$ ee. Compound $9 \mathbf{k}$ was found to be unstable to silica gel chromatograghy so the procedure was modified and the reaction was quenched with $\mathrm{H}_{2} \mathrm{O}$, and the organic layer was separated, dried over $\mathrm{MgSO}_{4}$, concentrated to deliver the product without the presence of impurities (see ${ }^{1} \mathrm{H}$ and ${ }^{13} \mathrm{C}$ NMR spectra of the crude reaction mixture in section 8). $\quad[\alpha]^{24}{ }_{\mathrm{D}}=-14.83\left(c 1.0, \mathrm{CHCl}_{3}, 82 \%\right.$ ee sample). The preparation of compound $9 \mathbf{k}$ was also carried out for $1 \mathrm{~h}$ at $-25^{\circ} \mathrm{C}$, generating 9k in 93\% yield $(24.7 \mathrm{mg}, 0.062 \mathrm{mmol})$ and 86\% ee. Enantiomeric excess was determined by HPLC with a Chiralcel OD-H column (99:1 nhexane:isopropanol, $0.5 \mathrm{~mL} / \mathrm{min}, 254 \mathrm{~nm}$ ); minor $t_{\mathrm{r}}=11.9 \mathrm{~min}$; major $\mathrm{t}_{\mathrm{r}}=12.6 \mathrm{~min}$. The absolute configuration was determined in analogy to compound $\mathbf{9 b}$.

Chromatograms of rac-9k and $9 \mathbf{k}$ :
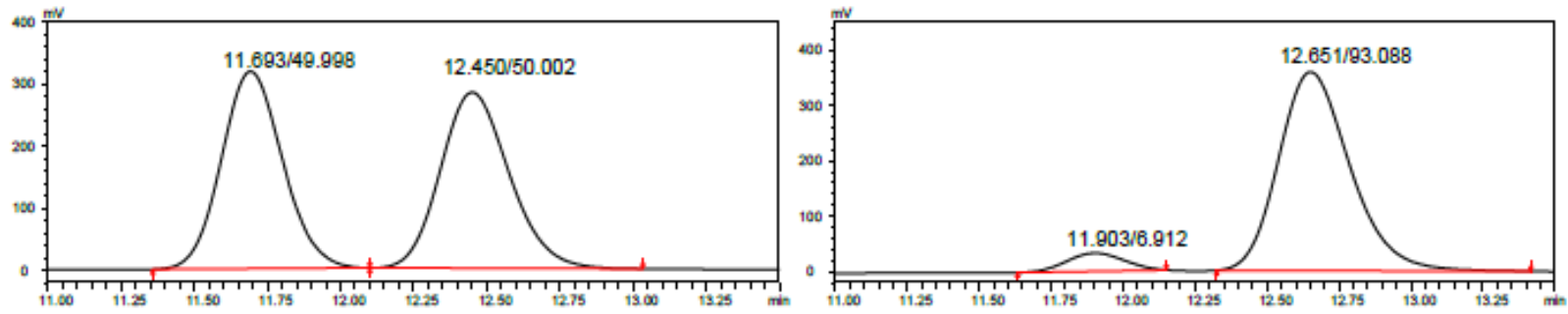

4-(1-phenyl-5-(trimethylsilyl)penta-1,4-diyn-3-yl)morpholine $\quad$ (rac-9l).

Compound rac-91 was prepared using procedure 3.1 with rac-StackPhos in Ph rac-91 TMS $73 \%$ yield $(30.2 \mathrm{mg}, 0.101 \mathrm{mmol})$ as a colorless oil. The reaction was carried out for $24 \mathrm{~h} . \mathrm{R}_{f}=0.53\left(20 \%\right.$ EtOAc in hexanes, $\mathrm{UV} / \mathrm{KMnO}_{4}$ stain). ${ }^{1} \mathrm{H} \mathrm{NMR}\left(500 \mathrm{MHz}, \mathrm{CDCl}_{3}\right)$ $\delta 7.56-7.43(\mathrm{~m}, 2 \mathrm{H}), 7.41-7.28(\mathrm{~m}, 3 \mathrm{H}), 4.58(\mathrm{~s}, 1 \mathrm{H}), 3.83(\mathrm{t}, J=4.7 \mathrm{~Hz}, 2 \mathrm{H}), 2.78(\mathrm{t}, J=4.7$ $\mathrm{Hz}, 2 \mathrm{H}), 0.24(\mathrm{~s}, 9 \mathrm{H}) .{ }^{13} \mathrm{C} \mathrm{NMR}\left(125 \mathrm{MHz}, \mathrm{CDCl}_{3}\right) \delta 132.1,128.7,128.4,122.6,98.9,90.2$, 
85.1, 83.3, 67.1, 50.2, 49.6, 0.1. HRMS (ESI-TOF) m/z: $[\mathrm{M}+\mathrm{H}]^{+}$Calcd for $\mathrm{C}_{18} \mathrm{H}_{24} \mathrm{NOSi}^{+}$ 298.1622; Found 298.1632.

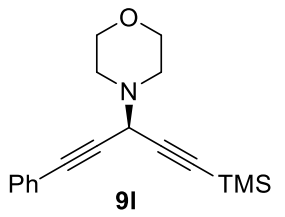

\section{(R)-4-(1-phenyl-5-(trimethylsilyl)penta-1,4-diyn-3-yl)morpholine}

(91).

Compound 91 was prepared using procedure 3.1 with $(S)$-StackPhos in $69 \%$ yield $(28.6 \mathrm{mg}, 0.096 \mathrm{mmol})$ as a colorless oil. The reaction was carried out for $24 \mathrm{~h} .[\alpha]^{24}=-72.98\left(c\right.$ 1.0, $\left.\mathrm{CHCl}_{3}\right)$. Enantiomeric excess was determined by HPLC with a Chiralcel OD-H column (99:1 $n$-hexane:isopropanol, $0.5 \mathrm{~mL} / \mathrm{min}, 215 \mathrm{~nm})$; $\operatorname{minor} \mathrm{t}_{\mathrm{r}}=10.5 \mathrm{~min}$; major $t_{r}=18.3 \mathrm{~min} ; 96 \%$ ee. The absolute configuration was determined in analogy to $\mathbf{9 b}$.

\section{Chromatograms of rac-9l and 91:}
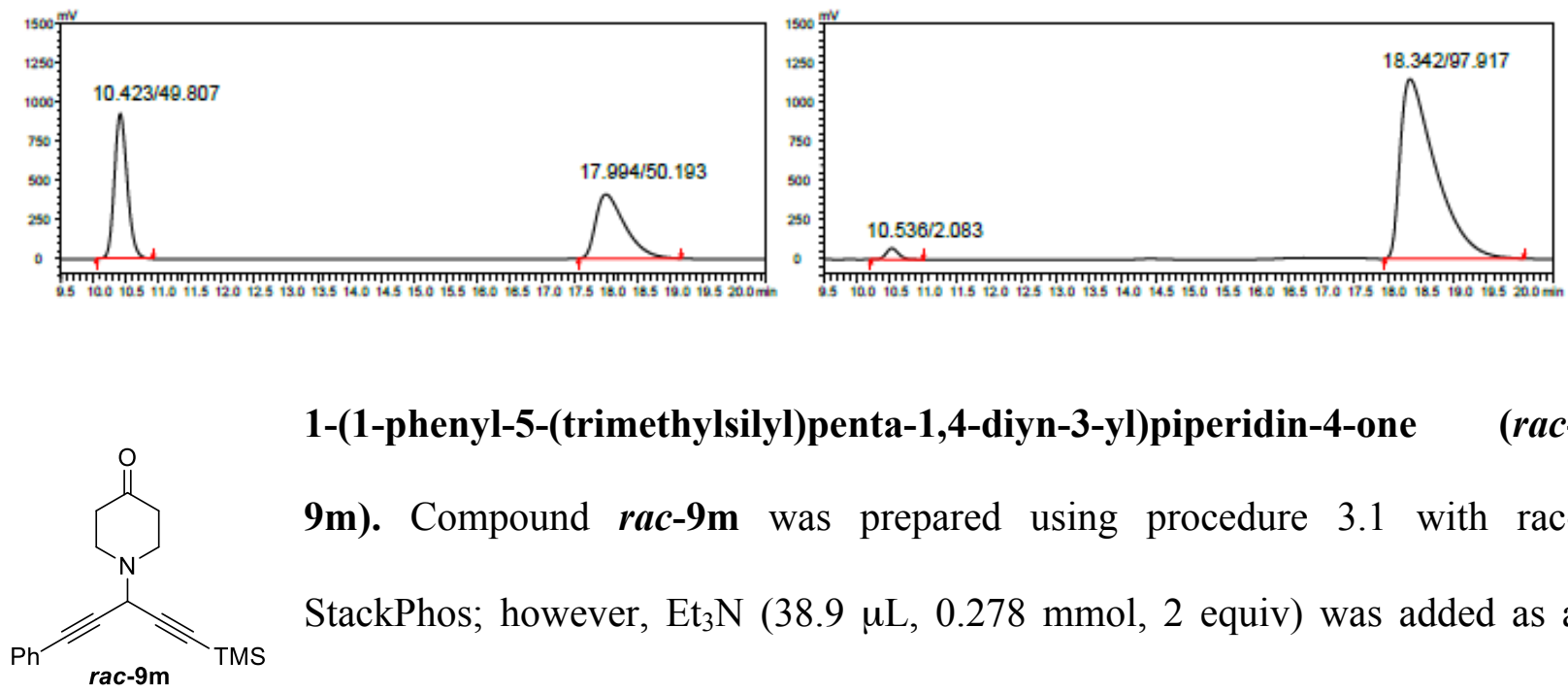

1-(1-phenyl-5-(trimethylsilyl)penta-1,4-diyn-3-yl)piperidin-4-one (rac-

9m). Compound rac-9m was prepared using procedure 3.1 with racStackPhos; however, $\mathrm{Et}_{3} \mathrm{~N}(38.9 \mu \mathrm{L}, 0.278 \mathrm{mmol}, 2$ equiv) was added as a final component to neutralize the 4-piperidone hydrate hydrochloride salt and deliver the product in $51 \%$ yield $(26.4 \mathrm{mg}, 0.085 \mathrm{mmol})$ as a yellowish oil. The reaction was carried out for $24 \mathrm{~h}$ at room temperature. $\mathrm{R}_{f}=0.38$ (20\% EtOAc in hexanes, $\mathrm{UV} / \mathrm{KMnO}_{4}$ stain). ${ }^{1} \mathrm{H}$ NMR $\left(500 \mathrm{MHz}, \mathrm{CDCl}_{3}\right) \delta 7.50-7.44(\mathrm{~m}, 2 \mathrm{H}), 7.36-7.28(\mathrm{~m}, 3 \mathrm{H}), 4.74(\mathrm{~s}, 1 \mathrm{H}), 3.03(\mathrm{t}, J=$ $6.1 \mathrm{~Hz}, 4 \mathrm{H}), 2.57(\mathrm{t}, J=6.2 \mathrm{~Hz}, 4 \mathrm{H}), 0.21(\mathrm{~s}, 9 \mathrm{H}) .{ }^{13} \mathrm{C} \mathrm{NMR}\left(125 \mathrm{MHz}, \mathrm{CDCl}_{3}\right) \delta 208.6,132.1$, 
128.9, 128.5, 122.4, 98.7, 90.2, 85.0, 83.2, 49.6, 49.4, 41.4, 0.1. HRMS (ESI-TOF) m/z: [M+H] ${ }^{+}$ Calcd for $\mathrm{C}_{19} \mathrm{H}_{24} \mathrm{NOSi}^{+}$310.1622; Found 310.1623.

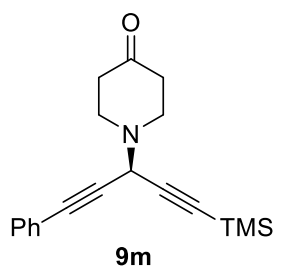

\section{(R)-1-(1-phenyl-5-(trimethylsilyl)penta-1,4-diyn-3-yl)piperidin-4-one}

(9m). Compound 9m was prepared using procedure 3.1 with $(S)$-StackPhos, however; however, $\mathrm{Et}_{3} \mathrm{~N}(38.9 \mu \mathrm{L}, 0.278 \mathrm{mmol}, 2$ equiv) was added as a final component to neutralize the 4-piperidone hydrate hydrochloride salt and deliver the product in $62 \%$ yield $(31.2 \mathrm{mg}, 0.101 \mathrm{mmol})$ as a yellowish oil. The reaction was carried out for $24 \mathrm{~h}$ at room temperature. $[\alpha]^{24}{ }_{\mathrm{D}}=+1.97\left(c 1.0, \mathrm{CHCl}_{3}\right)$. Enantiomeric excess was determined by HPLC with a Chiralcel OD-H column (99:1 $n$-hexane:isopropanol, $0.5 \mathrm{~mL} / \mathrm{min}$, $215 \mathrm{~nm}$ ); minor $t_{r}=19.4$ min; major $t_{r}=28.1$ min; $91 \%$ ee. The absolute configuration was determined in analogy to $\mathbf{9 b}$.

Chromatograms of rac-9m and 9m:
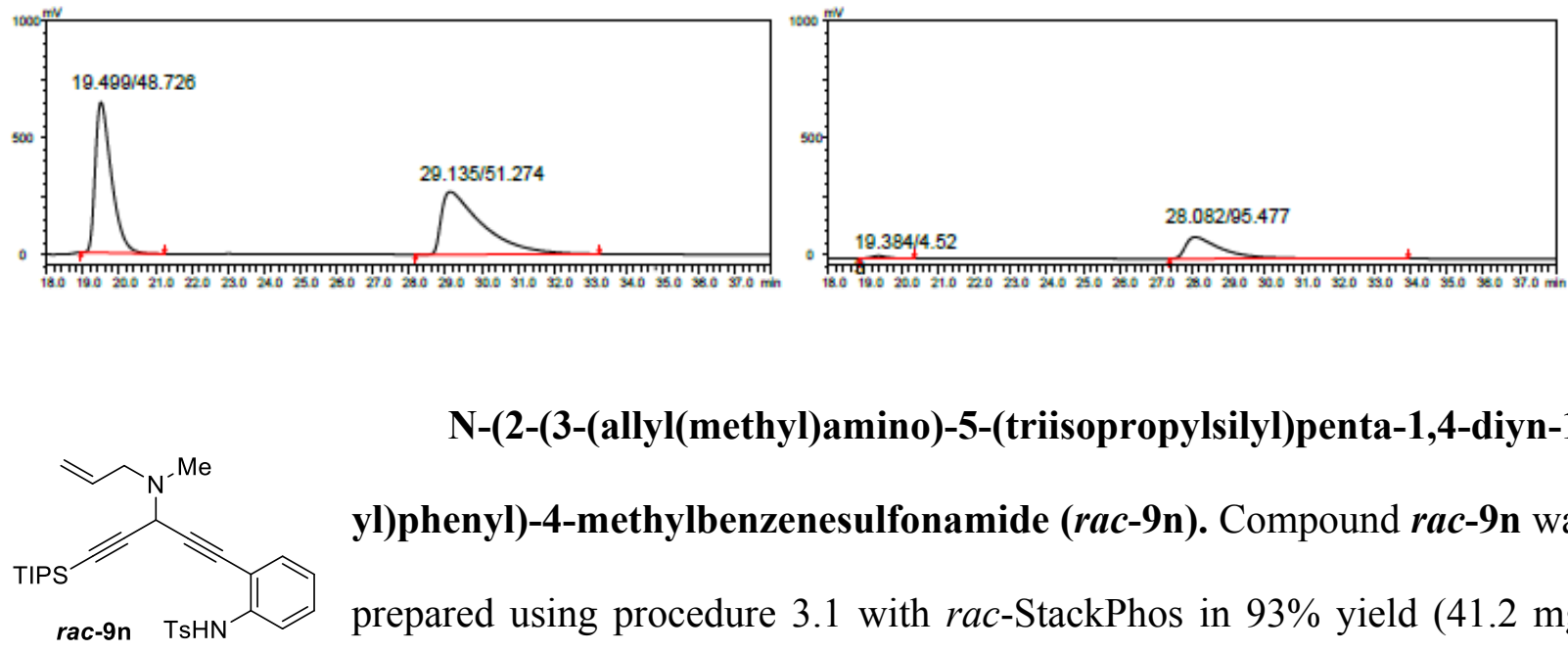

N-(2-(3-(allyl(methyl)amino)-5-(triisopropylsilyl)penta-1,4-diyn-1yl)phenyl)-4-methylbenzenesulfonamide (rac-9n). Compound rac-9n was

prepared using procedure 3.1 with rac-StackPhos in 93\% yield $(41.2 \mathrm{mg}$, $0.091 \mathrm{mmol})$ as a slightly red oil. The reaction was carried out for $3 \mathrm{~h} . \mathrm{R}_{f}=0.18(20 \%$ EtOAc in hexanes, UV/ $\mathrm{KMnO}_{4}$ stain). ${ }^{1} \mathrm{H} \mathrm{NMR}\left(500 \mathrm{MHz}, \mathrm{CDCl}_{3}\right) \delta 7.69(\mathrm{~d}, J=8.2 \mathrm{~Hz}, 2 \mathrm{H}), 7.60(\mathrm{~d}, J=$ $8.3 \mathrm{~Hz}, 1 \mathrm{H}), 7.35-7.15(\mathrm{~m}, 4 \mathrm{H}), 7.01(\mathrm{td}, J=7.6,1.2 \mathrm{~Hz}, 1 \mathrm{H}), 5.90$ (ddt, $J=16.8,10.2,6.7 \mathrm{~Hz}$, 1H), $5.30(\mathrm{dd}, J=17.3,2.0 \mathrm{~Hz}, 1 \mathrm{H}), 5.23(\mathrm{~d}, J=9.8 \mathrm{~Hz}, 1 \mathrm{H}), 4.70(\mathrm{~s}, 1 \mathrm{H}), 3.20-3.16(\mathrm{~m}, 1 \mathrm{H})$, 
$2.40(\mathrm{~s}, 3 \mathrm{H}), 2.36(\mathrm{~s}, 3 \mathrm{H}), 1.13(\mathrm{~s}, 21 \mathrm{H}) .{ }^{13} \mathrm{C}$ NMR $\left(125 \mathrm{MHz}, \mathrm{CDCl}_{3}\right) \delta$ 144.2, 138.2, 136.4, 135.3, 132.6, 129.9, 127.6, 124.3, 119.5, 118.9, 113.5, 110.2, 100.2, 92.1, 86.9, 78.8, 57.7, 48.3, 38.4, 21.7, 18.9, 11.4. HRMS (ESI-TOF) m/z: $[\mathrm{M}+\mathrm{H}]^{+}$Calcd for $\mathrm{C}_{31} \mathrm{H}_{43} \mathrm{~N}_{2} \mathrm{O}_{2}{ }^{+}$535.2809; Found 535.2813.

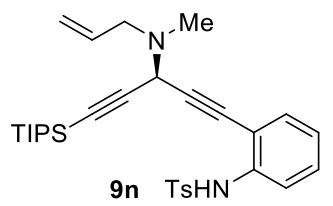

(S)-N-(2-(3-(allyl(methyl)amino)-5-(triisopropylsilyl)penta-1,4-diyn-1yl)phenyl)-4-methylbenzenesulfonamide (9n). Compound 9n was prepared using procedure 3.1 with $(S)$-StackPhos in $63 \%$ yield $(23.2 \mathrm{mg}$, $0.043 \mathrm{mmol})$ as a slightly red oil. The reaction was carried out for $3 \mathrm{~h} .[\alpha]^{24}{ }_{\mathrm{D}}=-14.38(c 1.0$, $\mathrm{CHCl}_{3}$ ). Enantiomeric excess was determined by HPLC with a Chiralcel OD-H column (99.9:0.1 $n$-hexane:isopropanol, $0.5 \mathrm{~mL} / \mathrm{min}, 254 \mathrm{~nm}$ ); $\operatorname{minor} \mathrm{t}_{\mathrm{r}}=107.4 \mathrm{~min}$; major $\mathrm{t}_{\mathrm{r}}=97.7 \mathrm{~min} ; 90 \%$ ee. The absolute configuration was determined in analogy to $\mathbf{9 b}$.

Chromatograms of rac-9n and 9n:
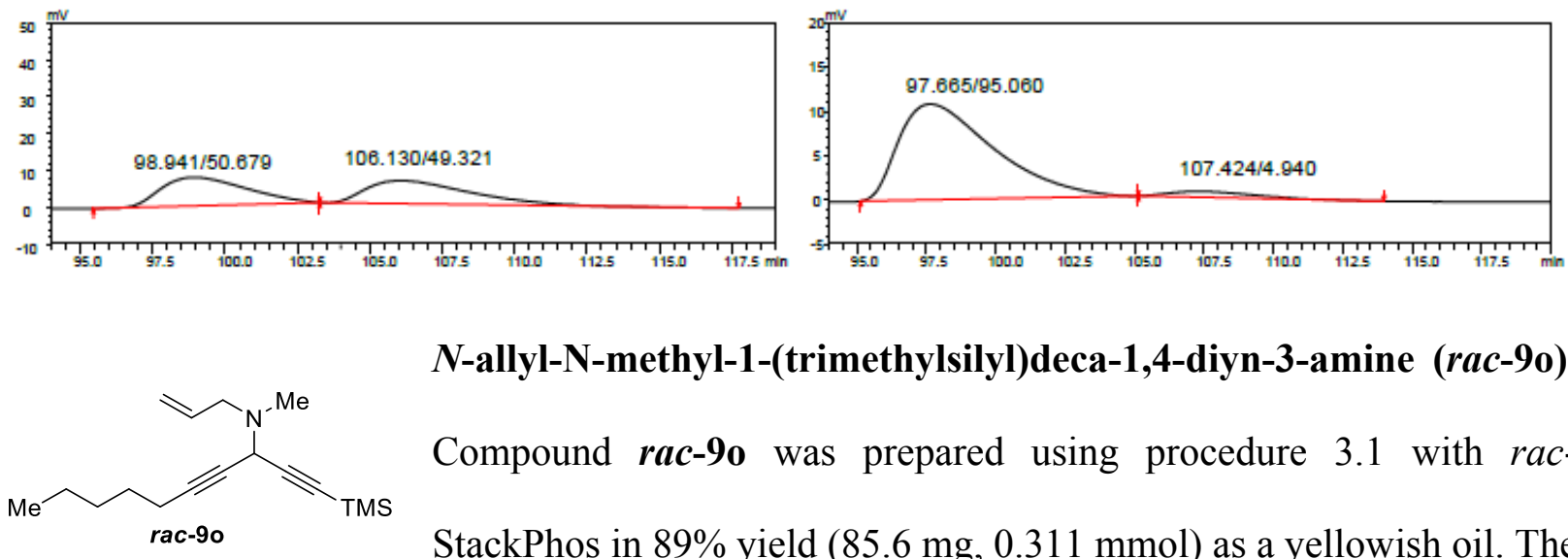

$N$-allyl-N-methyl-1-(trimethylsilyl)deca-1,4-diyn-3-amine (rac-9o).

Compound rac-9o was prepared using procedure 3.1 with racStackPhos in $89 \%$ yield $(85.6 \mathrm{mg}, 0.311 \mathrm{mmol})$ as a yellowish oil. The reaction was carried out for $3 \mathrm{~h} . \mathrm{R}_{f}=0.55\left(10 \%\right.$ EtOAc in hexanes, $\mathrm{UV} / \mathrm{KMnO}_{4}$ stain). ${ }^{1} \mathrm{H} \mathrm{NMR}$ $\left(500 \mathrm{MHz}, \mathrm{CDCl}_{3}\right) \delta 5.95-5.79(\mathrm{~m}, 1 \mathrm{H}), 5.24(\mathrm{~d}, J=17.2 \mathrm{~Hz}, 1 \mathrm{H}), 5.17(\mathrm{~d}, J=10.5 \mathrm{~Hz}, 1 \mathrm{H})$, $4.44(\mathrm{~d}, J=2.1 \mathrm{~Hz}, 1 \mathrm{H}), 3.12(\mathrm{~s}, 2 \mathrm{H}), 2.34(\mathrm{~s}, 3 \mathrm{H}), 2.27-2.20(\mathrm{~m}, 2 \mathrm{H}), 1.54(\mathrm{qt}, J=7.0,3.5 \mathrm{~Hz}$, 2H), $1.46-1.25(\mathrm{~m}, 4 \mathrm{H}), 0.98-0.84(\mathrm{~m}, 2 \mathrm{H}), 0.20(\mathrm{~s}, 9 \mathrm{H}) .{ }^{13} \mathrm{C}$ NMR $\left(125 \mathrm{MHz}, \mathrm{CDCl}_{3}\right) \delta$ 
$135.6,118.5,100.3,88.8,85.4,74.4,57.5,47.7,38.0,31.3,28.5,22.4,18.9,14.2,0.2$. HRMS (ESI-TOF) m/z: [M+H] ${ }^{+}$Calcd for $\mathrm{C}_{17} \mathrm{H}_{30} \mathrm{NSi}^{+}$276.2142; Found 276.2150 .

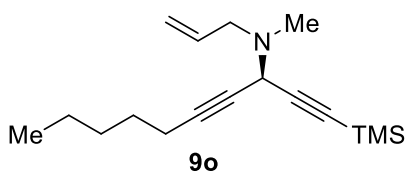

(R)- $N$-allyl-N-methyl-1-(trimethylsilyl)deca-1,4-diyn-3-amine

StackPhos in $71 \%$ yield $(22.6 \mathrm{mg}, 0.082 \mathrm{mmol})$ as a slightly red oil.

The reaction was carried out for $3 \mathrm{~h} .[\alpha]^{24}=-4.19\left(c\right.$ 1.0, $\left.\mathrm{CHCl}_{3}\right)$. Enantiomeric excess was determined to be $95 \%$ ee after Pauson-Khand reaction (see synthesis of $\mathbf{1 1}$ and chromatograms in section 4.2). The absolute configuration was determined in analogy to $\mathbf{9 b}$.

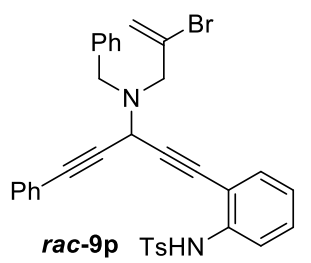

$N$-(2-(3-(benzyl(2-bromoallyl)amino)-5-phenylpenta-1,4-diyn-1yl)phenyl)-4-methylbenzenesulfonamide (rac-9p). Compound rac-9p was prepared using procedure 3.1 with $\mathrm{rac}$-StackPhos in $77 \%$ yield $(41.2 \mathrm{mg}$, $0.091 \mathrm{mmol}$ ) as a yellowish solid. The reaction was carried out for $3 \mathrm{~h} . \mathrm{R}_{f}=$ 0.39 (20\% EtOAc in hexanes, UV/ $\mathrm{KMnO}_{4}$ stain). ${ }^{1} \mathrm{H} \mathrm{NMR}\left(500 \mathrm{MHz}, \mathrm{CDCl}_{3}\right) \delta 7.67(\mathrm{~d}, J=8.0$ $\mathrm{Hz}, 3 \mathrm{H}), 7.59$ (dd, $J=6.7,3.0 \mathrm{~Hz}, 2 \mathrm{H}), 7.53(\mathrm{~d}, J=7.5 \mathrm{~Hz}, 2 \mathrm{H}), 7.45-7.27$ (m, 8H), $7.23-$ $7.17(\mathrm{~m}, 1 \mathrm{H}), 7.13-7.03(\mathrm{~m}, 3 \mathrm{H}), 6.10(\mathrm{~d}, J=2.2 \mathrm{~Hz}, 1 \mathrm{H}), 5.73(\mathrm{~s}, 1 \mathrm{H}), 4.87(\mathrm{~s}, 1 \mathrm{H}), 3.87(\mathrm{~s}$, 1H), $3.59(\mathrm{~s}, 1 \mathrm{H}), 2.31(\mathrm{~s}, 1 \mathrm{H}) .{ }^{13} \mathrm{C} \mathrm{NMR}\left(125 \mathrm{MHz}, \mathrm{CDCl}_{3}\right) \delta$ 144.1, 138.3, 137.9, 136.2, 132.5, $132.2,131.4,130.1,129.7,129.3,129.0,128.8,128.6,127.9,127.5,124.6,122.3,120.4,119.7$, 113.8, 91.6, 85.4, 83.0, 79.2, 59.4, 55.0, 45.3, 21.7. HRMS (ESI-TOF) m/z: $[\mathrm{M}+\mathrm{H}]^{+}$Calcd for $\mathrm{C}_{34} \mathrm{H}_{30} \mathrm{~N}_{2} \mathrm{O}_{2} \mathrm{SBr}^{+}$609.1206; Found 609.1186.

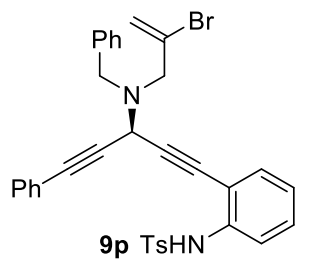

(R)-N-(2-(3-(benzyl(2-bromoallyl)amino)-5-phenylpenta-1,4-diyn-1yl)phenyl)-4-methylbenzenesulfonamide (9p). Compound $9 p$ was prepared using procedure 3.1 with $(S)$-StackPhos in 63\% yield (36.6 mg, $0.060 \mathrm{mmol}$ ) 
as a white solid. The reaction was carried out for $6 \mathrm{~h} .[\alpha]^{24}{ }_{\mathrm{D}}=-24.25$ (c 1.0, $\mathrm{CHCl}_{3}$ ). Enantiomeric excess was determined by HPLC with a Chiralcel OD-H column (98:2 nhexane:isopropanol, $1.0 \mathrm{~mL} / \mathrm{min}, 254 \mathrm{~nm}$ ); minor $\mathrm{t}_{\mathrm{r}}=15.5 \mathrm{~min}$; major $\mathrm{t}_{\mathrm{r}}=20.6 \mathrm{~min} ; 83 \%$ ee. The absolute configuration was determined in analogy to $\mathbf{9 b}$.

Chromatograms of rac-9p and 9p:
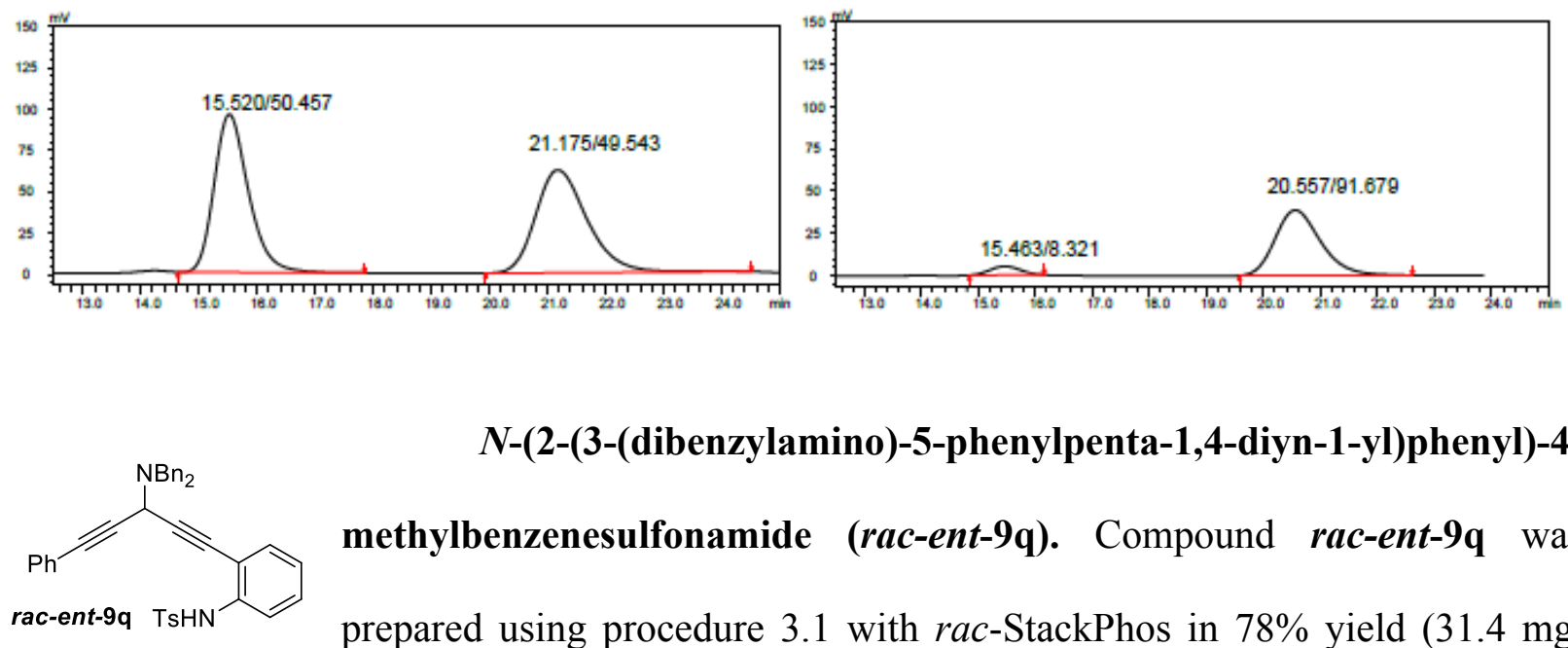

N-(2-(3-(dibenzylamino)-5-phenylpenta-1,4-diyn-1-yl)phenyl)-4methylbenzenesulfonamide (rac-ent-9q). Compound rac-ent-9q was prepared using procedure 3.1 with rac-StackPhos in $78 \%$ yield $(31.4 \mathrm{mg}$, $0.054 \mathrm{mmol})$ as a yellowish oil. The reaction was carried out for $3 \mathrm{~h} . \mathrm{R}_{f}=0.35(20 \%$ EtOAc in hexanes, $\mathrm{UV} / \mathrm{KMnO}_{4}$ stain). ${ }^{1} \mathrm{H} \mathrm{NMR}\left(500 \mathrm{MHz}, \mathrm{CDCl}_{3}\right) \delta 7.67(\mathrm{dd}, J=8.3,1.1 \mathrm{~Hz}, 1 \mathrm{H}), 7.64$ $(\mathrm{d}, J=8.3 \mathrm{~Hz}, 2 \mathrm{H}), 7.61-7.56(\mathrm{~m}, 2 \mathrm{H}), 7.51-7.47(\mathrm{~m}, 4 \mathrm{H}), 7.43-7.36(\mathrm{~m}, 7 \mathrm{H}), 7.37-7.26$ (m, 4H), $7.21(\mathrm{~s}, 1 \mathrm{H}), 7.08-7.00(\mathrm{~m}, 2 \mathrm{H}), 4.82(\mathrm{~s}, 1 \mathrm{H}), 3.88(\mathrm{~d}, J=13.5 \mathrm{~Hz}, 2 \mathrm{H}), 3.80(\mathrm{~d}, J=$ $13.5 \mathrm{~Hz}, 2 \mathrm{H}), 2.26(\mathrm{~s}, 3 \mathrm{H}) .{ }^{13} \mathrm{C} \mathrm{NMR}\left(125 \mathrm{MHz}, \mathrm{CDCl}_{3}\right) \delta 144.1,138.7,138.3,136.2,132.4$, $132.2,130.0,129.7,129.2,128.9,128.7,128.6,127.6,127.5,124.6,122.5,120.2,113.9,92.1$, 85.3, 83.7, 79.2, 55.3, 45.5, 21.7. HRMS (ESI-TOF) $\mathrm{m} / \mathrm{z}:[\mathrm{M}+\mathrm{H}]^{+}$Calcd for $\mathrm{C}_{38} \mathrm{H}_{33} \mathrm{~N}_{2} \mathrm{O}_{2} \mathrm{~S}^{+}$ 581.2257; Found 581.2253.

(R)-N-(2-(3-(dibenzylamino)-5-phenylpenta-1,4-diyn-1-yl)phenyl)-4-

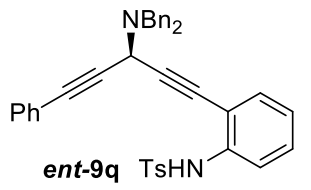

methylbenzenesulfonamide (ent-9q). Compound ent-9q was prepared using 
procedure 3.1 with $(S)$-StackPhos in $92 \%$ yield $(44.8 \mathrm{mg}, 0.077 \mathrm{mmol})$ as a yellowish oil. The reaction was carried out for $3 \mathrm{~h} .[\alpha]_{\mathrm{D}}^{24}=-26.23\left(c\right.$ 1.0, $\left.\mathrm{CHCl}_{3}\right)$. Enantiomeric excess was determined by HPLC with a Chiralcel OD-H column (99:1 $n$-hexane:isopropanol, $0.5 \mathrm{~mL} / \mathrm{min}$, $215 \mathrm{~nm}$ ); minor $t_{r}=38.7$ min; major $t_{r}=41.5$ min; $90 \%$ ee. The absolute configuration was determined in analogy to compound $\mathbf{9 b}$.

Chromatograms of rac-ent-9q and ent-9q:
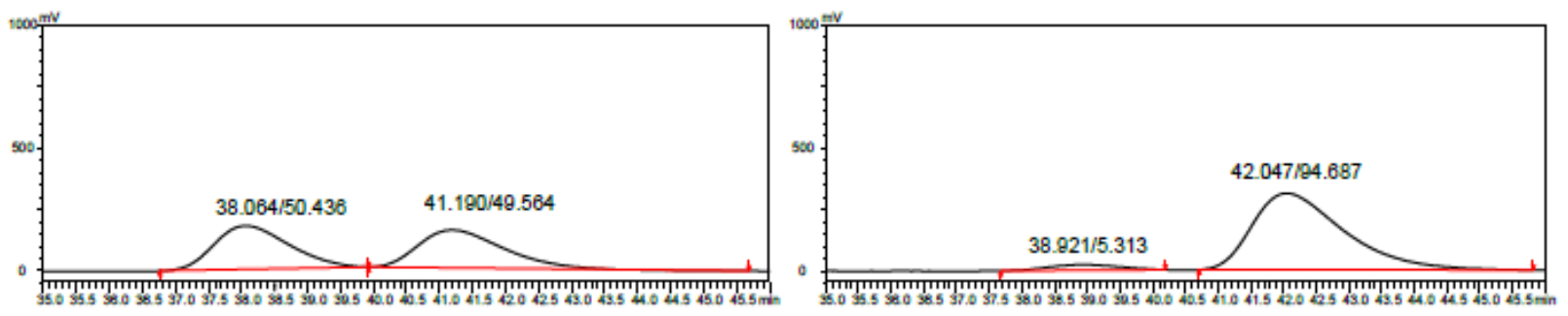

\section{Synthesis of Compounds 10, 11, 13, 14, 15, 16, 17 and 4}

4.1 Procedure for the Preparation of $\mathbf{1 0}$

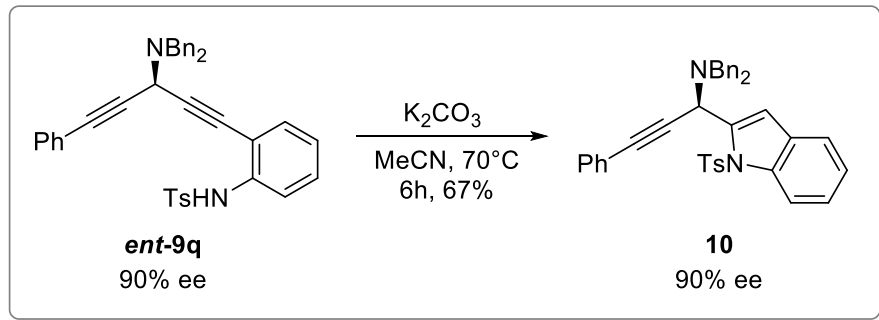

(R)-N,N-dibenzyl-3-phenyl-1-(1-tosyl-1H-indol-2-yl)prop-2-yn-1-amine (10). To a stirred solution of ent-9q (16.2 mg, $0.028 \mathrm{mmol}, 1.0$ equiv) in $\mathrm{MeCN}(1.0 \mathrm{~mL})$ was added $\mathrm{K}_{2} \mathrm{CO}_{3}\left(19.2 \mathrm{mg}, 0.140 \mathrm{mmol}, 5.0\right.$ equiv) and the mixture was stirred for $7 \mathrm{~h}$ at $70^{\circ} \mathrm{C}$. The solids were then filtered off and the solvent was evaporated under reduced pressure. The crude residue was subjected to flash column chromatography on silica gel ( $0-5 \%$ EtOAc in hexanes) to deliver $10(10.7 \mathrm{mg}, 0.019 \mathrm{mmol})$ in $67 \%$ yield as a white oil. $\mathrm{R}_{f}=0.50(20 \%$ EtOAc in hexanes, UV/ $\mathrm{KMnO}_{4}$ stain). ${ }^{1} \mathrm{H}$ NMR $\left(500 \mathrm{MHz}, \mathrm{CDCl}_{3}\right) \delta 8.03(\mathrm{~d}, J=8.4 \mathrm{~Hz}, 1 \mathrm{H}), 7.55(\mathrm{dd}, J=6.6,3.0 \mathrm{~Hz}$, 
2H), $7.49(\mathrm{~d}, J=8.3 \mathrm{~Hz}, 2 \mathrm{H}), 7.42-7.32(\mathrm{~m}, 7 \mathrm{H}), 7.29-7.20(\mathrm{~m}, 5 \mathrm{H}), 7.16(\mathrm{td}, J=7.4,3.3 \mathrm{~Hz}$, 3H), $7.08(\mathrm{~d}, J=8.1 \mathrm{~Hz}, 2 \mathrm{H}), 7.01(\mathrm{~s}, 1 \mathrm{H}), 5.92(\mathrm{~s}, 1 \mathrm{H}), 3.96(\mathrm{~d}, J=13.7 \mathrm{~Hz}, 2 \mathrm{H}), 3.75(\mathrm{~d}, J=$ $13.7 \mathrm{~Hz}, 2 \mathrm{H}), 2.31(\mathrm{~s}, 3 \mathrm{H}) .{ }^{13} \mathrm{C} \mathrm{NMR}\left(125 \mathrm{MHz}, \mathrm{CDCl}_{3}\right) \delta 144.6,139.5,139.0,138.2,135.9$, 132.1, 129.7, 129.6, 129.2, 128.6, 128.2, 127.0, 126.8, 124.9, 123.8, 123.5, 123.2, 121.1, 115.7, 114.3, 87.4, 85.3, 55.8, 52.9, 21.8. $[\alpha]^{24}=-32.08\left(c\right.$ 1.0, $\left.\mathrm{CHCl}_{3}\right)$. HRMS (ESI-TOF) $\mathrm{m} / \mathrm{z}$ : $[\mathrm{M}+\mathrm{H}]^{+}$Calcd for $\mathrm{C}_{38} \mathrm{H}_{33} \mathrm{~N}_{2} \mathrm{O}_{2} \mathrm{~S}^{+}$581.2257; Found 581.2253. Enantiomeric excess was determined by HPLC with a Chiralcel OD-H column (99:1 $n$-hexane:isopropanol, $0.5 \mathrm{~mL} / \mathrm{min}$, $254 \mathrm{~nm}$ ); minor $t_{r}=47.1$ min; major $t_{r}=31.1 \mathrm{~min}$; $90 \%$ ee. The absolute configuration was determined in analogy to ent-9q.

Chromatograms of rac-10 and 10:
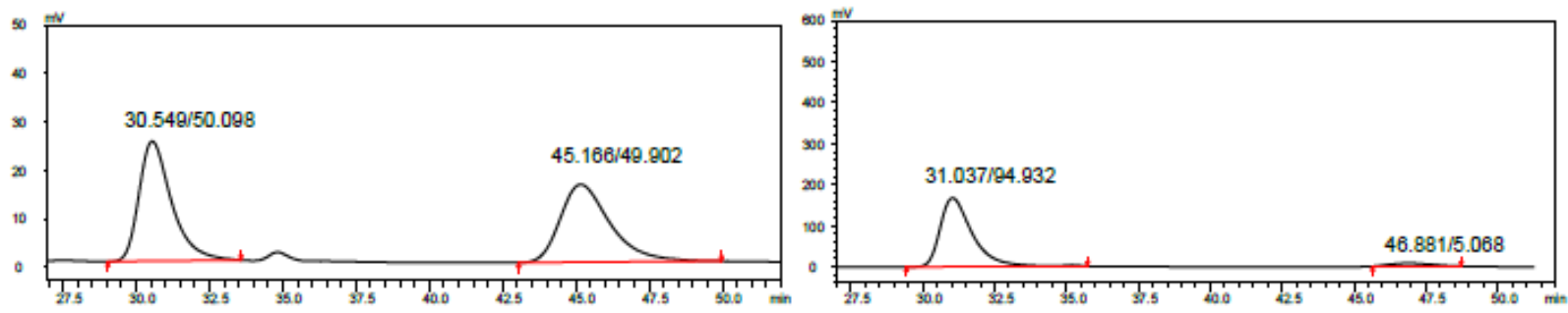

4.2 Procedure for the Preparation of $\mathbf{1 1}$

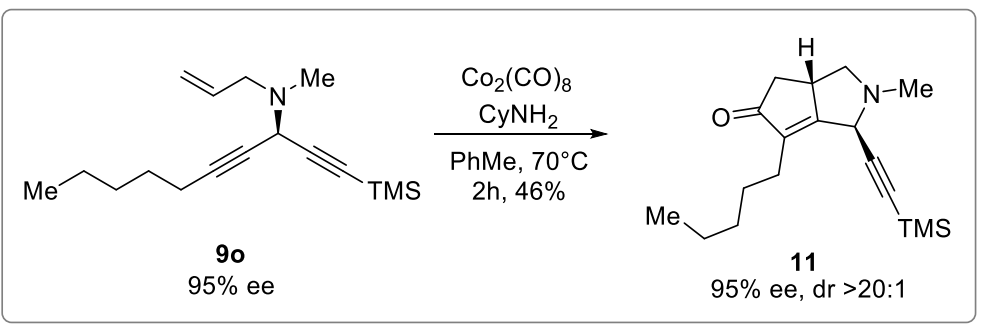

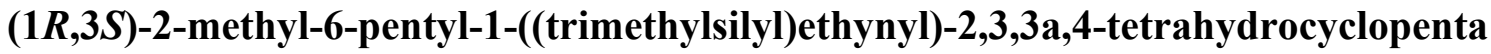

[c]pyrrol-5(1H)-one (11). $\mathrm{Co}_{2}(\mathrm{CO})_{8}(26.0 \mathrm{mg}, 0.076 \mathrm{mmol}, 2.0$ equiv) and cyclohexylamine $(25.0 \mu \mathrm{L}, 0.190 \mathrm{mmol}, 5.0$ equiv) were added to a stirred solution of 90 1.0 equiv) in $\mathrm{PhMe}(0.6 \mathrm{~mL})$. The mixture was stirred for $2 \mathrm{~h}$ at $70^{\circ} \mathrm{C}$ and the reaction mixture 
was then directly subjected to flash column chromatography on silica gel $(0-5 \%$ EtOAc in hexanes) to deliver $11(5.3 \mathrm{mg}, 0.017 \mathrm{mmol})$ in $46 \%$ yield as a brownish oil. $\mathrm{R}_{f}=0.34(10 \%$ EtOAc in hexanes, UV/ $\mathrm{KMnO}_{4}$ stain). ${ }^{1} \mathrm{H} \mathrm{NMR}\left(500 \mathrm{MHz}, \mathrm{CDCl}_{3}\right) \delta 4.44(\mathrm{~s}, 1 \mathrm{H}), 3.50(\mathrm{t}, J=$ $7.5 \mathrm{~Hz}, 1 \mathrm{H}), 3.32(\mathrm{q}, J=8.0 \mathrm{~Hz}, 1 \mathrm{H}), 2.64(\mathrm{ddd}, J=18.4,6.5,1.9 \mathrm{~Hz}, 1 \mathrm{H}), 2.60(\mathrm{~s}, 3 \mathrm{H}), 2.44-$ $2.33(\mathrm{~m}, 1 \mathrm{H}), 2.24-2.10(\mathrm{~m}, 2 \mathrm{H}), 2.01(\mathrm{dd}, J=10.7,8.5 \mathrm{~Hz}, 1 \mathrm{H}), 1.62-1.37(\mathrm{~m}, 4 \mathrm{H}), 1.38-$ $1.23(\mathrm{~m}, 4 \mathrm{H}), 0.90(\mathrm{td}, J=7.2,1.9 \mathrm{~Hz}, 3 \mathrm{H}), 0.33(\mathrm{~s}, 9 \mathrm{H}) .{ }^{13} \mathrm{C} \mathrm{NMR}\left(125 \mathrm{MHz}, \mathrm{CDCl}_{3}\right) \delta 209.2$, $176.4,137.4,112.5,79.6,66.4,61.2,42.7,41.7,39.7,32.1,27.2,24.8,22.6,14.2,1.1 .[\alpha]^{24}=$ $+4.67\left(c\right.$ 0.5, $\left.\mathrm{CHCl}_{3}\right)$. HRMS (ESI-TOF) m/z: $[\mathrm{M}+\mathrm{H}]^{+}$Calcd for $\mathrm{C}_{18} \mathrm{H}_{30} \mathrm{NOSi}^{+}$304.2091; Found 304.2099. Enantiomeric excess was determined by HPLC with a Chiralcel OD-H column (99:1 $n$-hexane:isopropanol, $1.0 \mathrm{~mL} / \mathrm{min}, 254 \mathrm{~nm}$ ); minor $\mathrm{t}_{\mathrm{r}}=3.9 \mathrm{~min}$; major $\mathrm{t}_{\mathrm{r}}=4.7 \mathrm{~min}$; $95 \%$ ee. The diastereoselectivity was determined by ${ }^{1} \mathrm{H}$ NMR, where only one diastereomer was observable. The absolute configuration was determined in analogy to 9o, and the relative stereochemistry in analogy to a similar compound prepared by Knochel and coworkers. ${ }^{7}$

Chromatograms of rac-11 and 11:
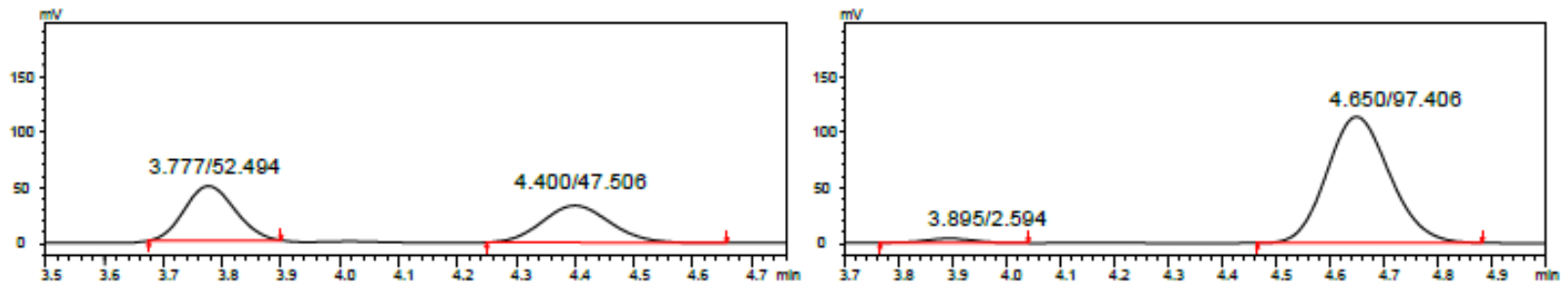


\subsection{Procedure for the Preparation of $\mathbf{1 3}, \mathbf{1 4}$ and $\mathbf{1 5}$}

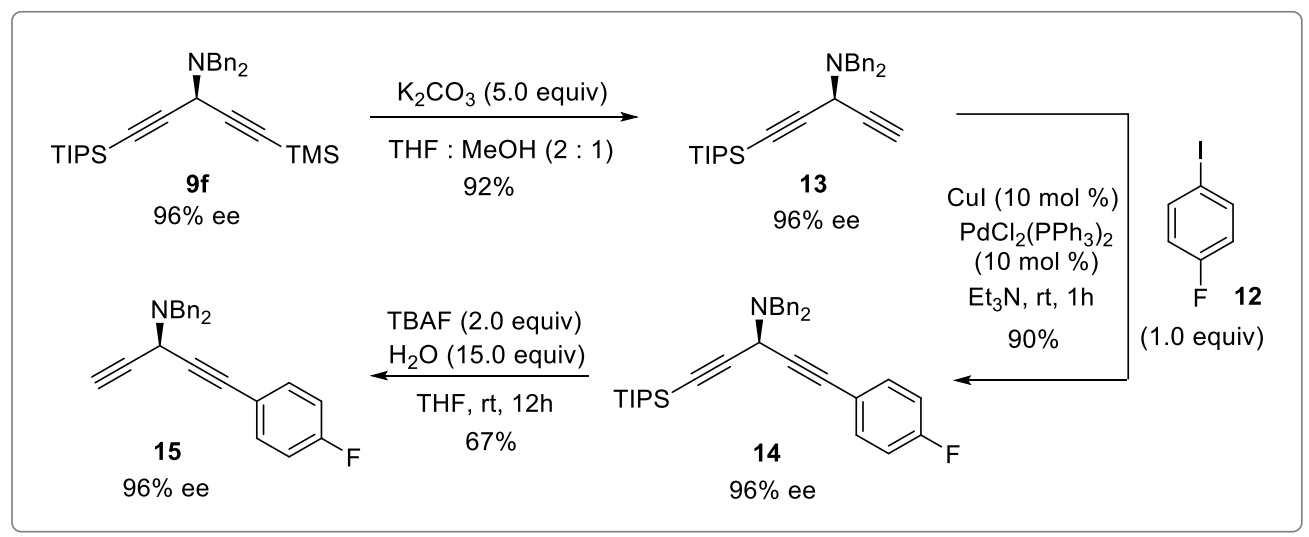

(S)- $N, N$-dibenzyl-1-(triisopropylsilyl)penta-1,4-diyn-3-amine (13). $\mathrm{K}_{2} \mathrm{CO}_{3}(33.9 \mathrm{mg}$,

$0.246 \mathrm{mmol}, 5.0$ equiv) was added to a stirred solution of $9 \mathbf{f}(24.0 \mathrm{mg}, 0.049 \mathrm{mmol}, 1.0$ equiv) in THF : $\mathrm{MeOH}(2: 1$ mixture, $1.5 \mathrm{~mL}$ total) and the mixture was stirred for $1 \mathrm{~h}$ at room temperature. The reaction was quenched with $1 \mathrm{M} \mathrm{HCl}(0.5 \mathrm{~mL})$ and diluted with $\mathrm{CH}_{2} \mathrm{Cl}_{2}(5.0$ $\mathrm{mL})$ and $\mathrm{H}_{2} \mathrm{O}(2.0 \mathrm{~mL})$. The organic layer was separated, dried over $\mathrm{MgSO}$, filtered and concentrated under reduced pressure to afford a residue that was purified by flash column chromatography on silica gel (0-5\% EtOAc in hexanes) to deliver $13(18.9 \mathrm{mg}, 0.045 \mathrm{mmol})$ in $92 \%$ yield as a colorless oil. $\mathrm{R}_{f}=0.73\left(20 \%\right.$ EtOAc in hexanes, UV/ $\mathrm{KMnO}_{4}$ stain $) .{ }^{1} \mathrm{H}$ NMR $\left(500 \mathrm{MHz}, \mathrm{CDCl}_{3}\right) \delta 7.43(\mathrm{~d}, J=7.5 \mathrm{~Hz}, 4 \mathrm{H}), 7.37-7.31(\mathrm{~m}, 4 \mathrm{H}), 7.30-7.23(\mathrm{~m}, 2 \mathrm{H}), 4.41(\mathrm{~d}$, $J=2.4 \mathrm{~Hz}, 1 \mathrm{H}), 3.83(\mathrm{~d}, J=13.5 \mathrm{~Hz}, 2 \mathrm{H}), 3.72(\mathrm{~d}, J=13.4 \mathrm{~Hz}, 2 \mathrm{H}), 2.39(\mathrm{~d}, J=2.4 \mathrm{~Hz}, 1 \mathrm{H})$, $1.15(\mathrm{~s}, 21 \mathrm{H}) .{ }^{13} \mathrm{C} \mathrm{NMR}\left(125 \mathrm{MHz}, \mathrm{CDCl}_{3}\right) \delta 139.8,129.9,129.5,128.4,103.1,85.9,79.7,74.2$, 55.5, 45.5, 19.3, 12.2. $[\alpha]^{24}{ }_{\mathrm{D}}=-64.34\left(c 1.0, \mathrm{CHCl}_{3}\right)$. HRMS (ESI-TOF) $\mathrm{m} / \mathrm{z}:[\mathrm{M}+\mathrm{H}]^{+}$Calcd for $\mathrm{C}_{28} \mathrm{H}_{38} \mathrm{NSi}^{+}$416.2768; Found 416.2771. Enantiomeric excess was determined by HPLC with a Chiralcel OD-H column (100:0 $n$-hexane:isopropanol, $0.2 \mathrm{~mL} / \mathrm{min}, 215 \mathrm{~nm})$; minor $\mathrm{t}_{\mathrm{r}}=22.5$ $\min$; major $\mathrm{t}_{\mathrm{r}}=23.5 \mathrm{~min} ; 96 \%$ ee. The absolute configuration was determined in analogy to $\mathbf{9 f}$. 
(S)-N,N-dibenzyl-1-(4-fluorophenyl)-5-(triisopropylsilyl)penta-1,4-diyn-3-amine(14).

$\mathrm{CuI}(0.6 \mathrm{mg}, 0.0031 \mathrm{mmol}, 10 \mathrm{~mol} \%)$ and $\mathrm{PdCl}_{2}\left(\mathrm{PPh}_{3}\right)_{2}(2.2 \mathrm{mg}, 0.0031 \mathrm{mmol}, 10 \mathrm{~mol} \%)$ were added to a stirred solution of 4-fluoro-1-iodobenzene (3.6 $\mu \mathrm{L}, 0.031 \mathrm{mmol}, 1.0$ equiv) and $\mathbf{1 3}$ (13.0 mg, $0.031 \mathrm{mmol}, 1.0$ equiv) in $\mathrm{Et}_{3} \mathrm{~N}(0.15 \mathrm{~mL}, 1.25 \mathrm{mmol}, 40.0$ equiv), at room temperature. The mixture was stirred for $1 \mathrm{~h}$, concentrated under reduced pressure and submitted to flash colum chromatography on silica gel (2\% EtOAc in hexanes) to afford 14 (14.3 mg, 0.028 mmol) in $90 \%$ yield as a yellowish oil. $\mathrm{R}_{f}=0.79\left(10 \%\right.$ EtOAc in hexanes, $\mathrm{UV} / \mathrm{KMnO}_{4}$ stain). ${ }^{1} \mathrm{H}$ NMR $\left(500 \mathrm{MHz}, \mathrm{CDCl}_{3}\right) \delta 7.44(\mathrm{dd}, J=8.1,5.8 \mathrm{~Hz}, 6 \mathrm{H}), 7.35(\mathrm{t}, J=7.5 \mathrm{~Hz}, 4 \mathrm{H}), 7.31-7.23$ (m, 2H), 7.02 (t, $J=8.7 \mathrm{~Hz}, 2 \mathrm{H}), 4.60(\mathrm{~s}, 1 \mathrm{H}), 3.89$ (d, $J=13.4 \mathrm{~Hz}, 2 \mathrm{H}), 3.75(\mathrm{~d}, J=13.4 \mathrm{~Hz}$, 2H), $1.16(\mathrm{~s}, 21 \mathrm{H}) .{ }^{13} \mathrm{C}$ NMR $\left(125 \mathrm{MHz}, \mathrm{CDCl}_{3}\right) \delta 163.7,161.72,139.2,134.0,129.2,128.5$, $127.4,119.2,115.8,115.6,102.0,85.7,85.1,82.8,55.1,45.6,18.9,11.5 .[\alpha]^{24}=-9.96(c 1.0$, $\mathrm{CHCl}_{3}$ ). HRMS (ESI-TOF) m/z: [M+H] $]^{+}$Calcd for $\mathrm{C}_{34} \mathrm{H}_{41} \mathrm{NSiF}^{+}$510.2987; Found 510.2986. Enantiomeric excess was determined by HPLC with a Chiralcel OD-H column (100:0 nhexane:isopropanol, $1.0 \mathrm{~mL} / \mathrm{min}, 254 \mathrm{~nm}$ ); minor $t_{r}=5.8$ min; major $t_{r}=6.3 \mathrm{~min} ; 96 \%$ ee. The absolute configuration was determined in analogy to $9 \mathbf{f}$.

(R)- $N, N$-dibenzyl-1-(4-fluorophenyl)penta-1,4-diyn-3-amine (15). $\quad \mathrm{H}_{2} \mathrm{O} \quad(1.6 \mu \mathrm{L}$, $0.0882 \mathrm{mmol}, 15.0$ equiv) and TBAF (11.8 $\mu \mathrm{L}, 1 \mathrm{M}$ in THF, $0.0118 \mathrm{mmol}, 2.0$ equiv) were added to a stirred solution of $14(3.0 \mathrm{mg}, 0.0059 \mathrm{mmol}, 1.0$ equiv) in THF (0.3 mL) and the mixture was stirred for $14 \mathrm{~h}$ at room temperature. The reaction was quenched with $\mathrm{H}_{2} \mathrm{O}(0.5 \mathrm{~mL})$ and EtOAc (5.0 mL). The organic layer was separated, dried over MgSO4, filtered and concentrated under reduced pressure to give a residue which was purified through flash column chromatography on silica gel (0-5\% EtOAc in hexanes) to afford 15 (1.4 $\mathrm{mg}, 0.0040 \mathrm{mmol})$ in $67 \%$ yield as a colorless oil. $\mathrm{R}_{f}=0.58\left(10 \%\right.$ EtOAc in hexanes, $\mathrm{UV} / \mathrm{KMnO}_{4}$ stain). ${ }^{1} \mathrm{H} \mathrm{NMR}$ 
$\left(500 \mathrm{MHz}, \mathrm{CDCl}_{3}\right) \delta 7.50-7.42(\mathrm{~m}, 6 \mathrm{H}), 7.35(\mathrm{t}, J=7.4 \mathrm{~Hz}, 4 \mathrm{H}), 7.29(\mathrm{t}, J=8.7 \mathrm{~Hz}, 2 \mathrm{H}), 7.02$ $(\mathrm{t}, J=8.7 \mathrm{~Hz}, 2 \mathrm{H}), 4.62(\mathrm{~s}, 1 \mathrm{H}), 3.84(\mathrm{q}, J=14.6 \mathrm{~Hz}, 4 \mathrm{H}) 2.49(\mathrm{~s}, 1 \mathrm{H}) \cdot[\alpha]^{24}{ }_{\mathrm{D}}=-14.78(c 0.2$, $\mathrm{CHCl}_{3}$ ). HRMS (ESI-TOF) m/z: $[\mathrm{M}+\mathrm{H}]^{+}$Calcd for $\mathrm{C}_{25} \mathrm{H}_{21} \mathrm{NF}^{+}$354.1653; Found 354.1660. Enantiomeric excess was determined by HPLC with a Chiralcel OD-H column (100:0 nhexane:isopropanol, $1.0 \mathrm{~mL} / \mathrm{min}, 254 \mathrm{~nm}$ ); minor $\mathrm{t}_{\mathrm{r}}=33.4 \mathrm{~min}$; major $\mathrm{t}_{\mathrm{r}}=18.8 \mathrm{~min}$; $96 \%$ ee. The absolute configuration was determined in analogy to $9 \mathbf{f}$.

Chromatograms of rac-13 and 13:
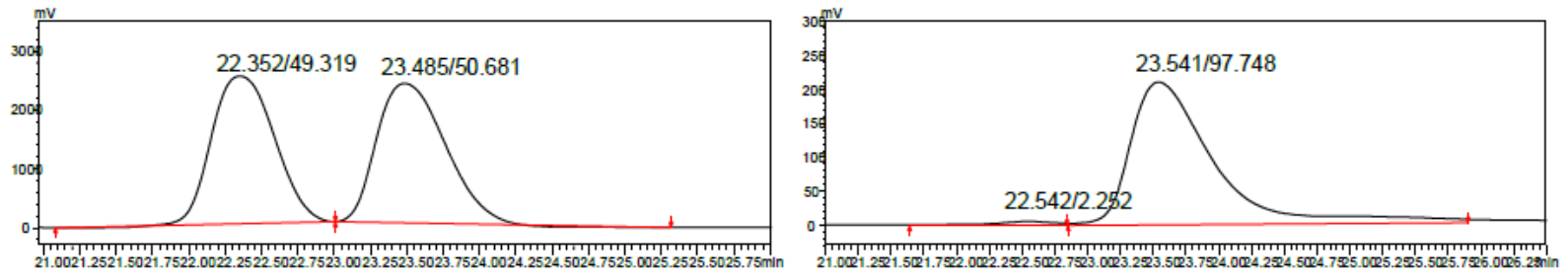

Chromatograms of rac-14 and 14:
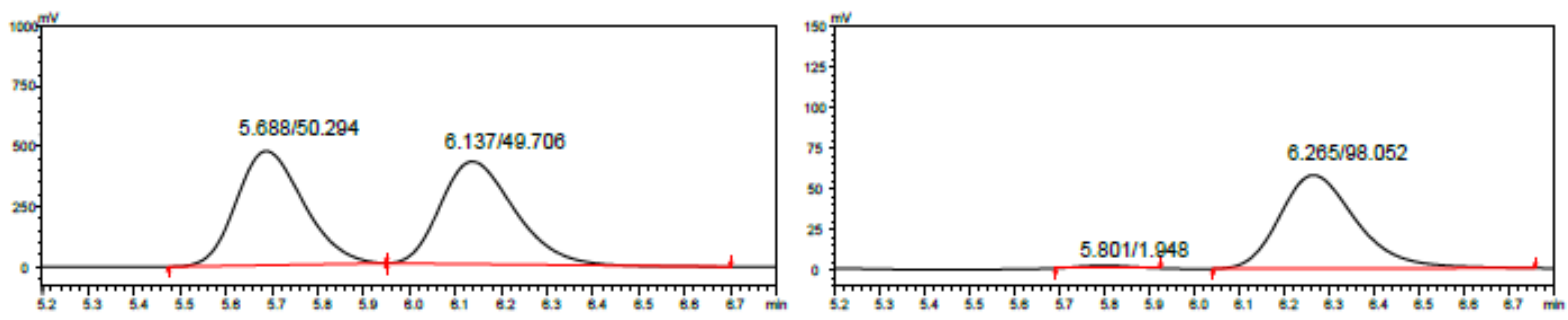

Chromatograms of rac-15 and $\mathbf{1 5}$ :
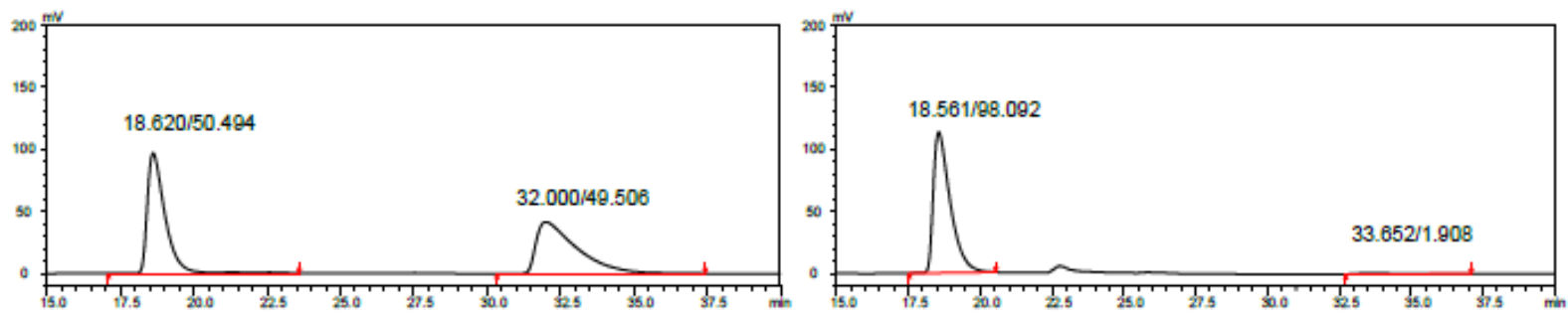
4.4 Procedure for the Preparation of 16, 17, and 4

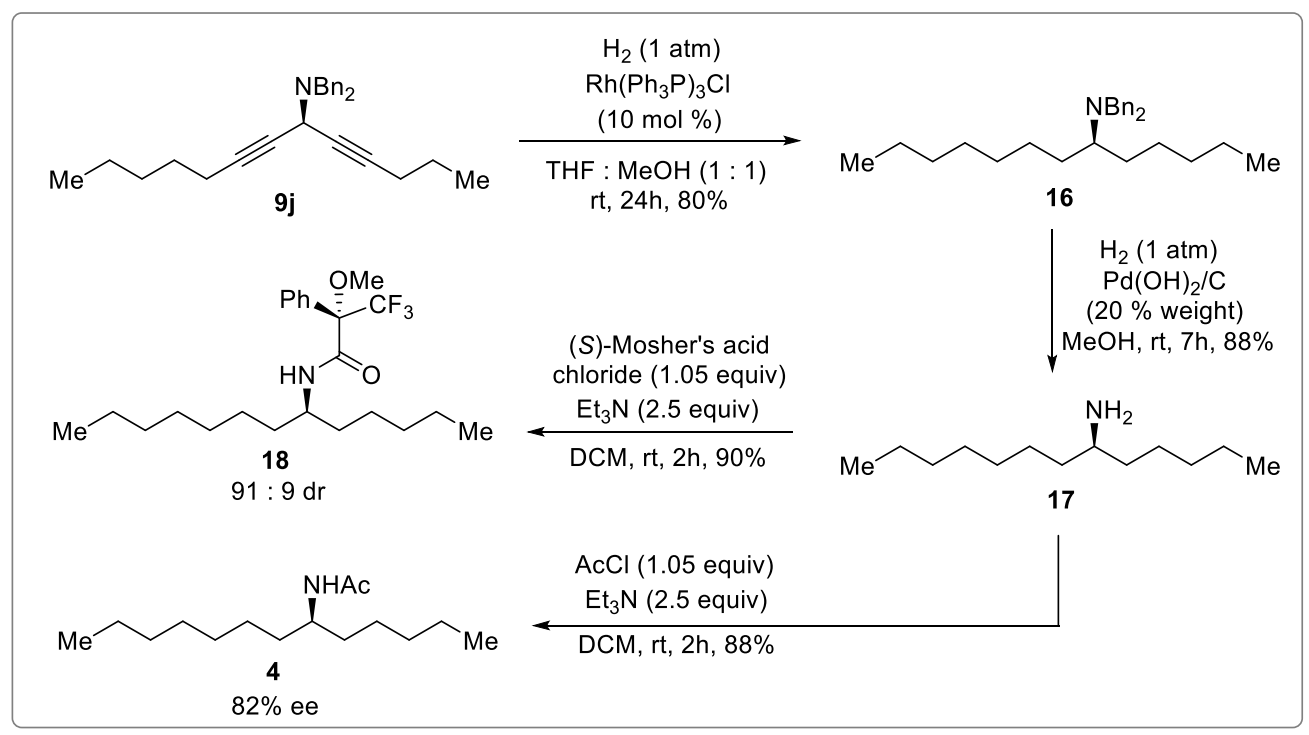

(S)-N,N-dibenzyltridecan-6-amine (16). $\left(\mathrm{Ph}_{3} \mathrm{P}\right)_{3} \mathrm{RhCl}(0.0050$ g, 0.0054 mmol, 0.1 equiv) and a magnetic stir bar were added to a flask which was capped with a rubber septum. A mixture of THF : MeOH $(1: 1,0.4 \mathrm{~mL}$ total) was added to the flask hydrogen was bubbled through the reaction mixture for $5 \mathrm{~min}$ via a hydrogen ballon. A solution of $\mathbf{9 j}(0.0202 \mathrm{~g}, 0.054$ mmol, 1.0 equiv) in THF : MeOH $(1: 1,0.4 \mathrm{~mL}$ total) was then added to the solution containing the catalyst and hydrogen, and the reaction mixture was stirred for $24 \mathrm{~h}$. The reaction was adsorbed onto silica gel and purified using a silica gel plug (3\% EtOAc in hexanes) to afford $\mathbf{1 6}$ $(0.0165 \mathrm{mg}, 0.0435 \mathrm{mmol})$ in $80 \%$ yield as a colorless oil. $\mathrm{R}_{f}=0.90(20 \%$ EtOAc in hexanes, $\mathrm{UV} / \mathrm{KMnO}_{4}$ stain). ${ }^{1} \mathrm{H} \mathrm{NMR}\left(500 \mathrm{MHz}, \mathrm{CDCl}_{3}\right) \delta$ 7.37-7.32 (m, 4H), 7.30-7.24 (m, 2H), 7.22$7.16(\mathrm{~m}, 2 \mathrm{H}), 3.54(\mathrm{~s}, 1 \mathrm{H}), 2.42-2.35(\mathrm{~m}, 1 \mathrm{H}), 1.63-1.43(\mathrm{~m}, 2 \mathrm{H}), 1.43-1.04(\mathrm{~m}, 18 \mathrm{H}), 0.88$ $(\mathrm{t}, J=7.1 \mathrm{~Hz}, 3 \mathrm{H}), 0.85(\mathrm{t}, J=7.1 \mathrm{~Hz}, 3 \mathrm{H}) .{ }^{13} \mathrm{C} \mathrm{NMR}\left(125 \mathrm{MHz}, \mathrm{CDCl}_{3}\right) \delta 141.2,129.1,128.2$, $126.8,57.3,53.6,32.3,32.2,30.0,29.9,29.9,29.6,27.4,27.1,22.9,14.4,14.3 \cdot[\alpha]^{24}=-13.76(c$ 1.0, $\mathrm{CHCl}_{3}$ ). HRMS (ESI-TOF) m/z: $[\mathrm{M}+\mathrm{H}]^{+}$Calcd for $\mathrm{C}_{27} \mathrm{H}_{42} \mathrm{~N}^{+}$380.3312; Found 380.3343 . 
(S)-tridecan-6-amine (17). $\mathrm{Pd}(\mathrm{OH})_{2} / \mathrm{C}(0.0021 \mathrm{~g}, 10 \%$ palladium content, $20 \%$ weight in relation to substrate) and a magnetic stir bar were added to a flask and a solution of $\mathbf{1 6}(0.0105$ g, $0.028 \mathrm{mmol}, 1.0$ equiv) in $\mathrm{MeOH}(0.8 \mathrm{~mL})$ was then added. The reaction mixture was stirred for $7 \mathrm{~h}$ under $1 \mathrm{~atm}$ atmosphere of hydrogen (via a hydrogen) then filtered through a plug of celite with EtOAc $(3.0 \mathrm{~mL})$. The solvent was removed under reduced pressure to afford $\mathbf{1 7}$ $(0.0049 \mathrm{~g}, 0.025 \mathrm{mmol})$ in $88 \%$ yield as a colorless oil. $\mathrm{R}_{f}=0.0(20 \%$ EtOAc in hexanes, UV/ $\mathrm{KMnO}_{4}$ stain). ${ }^{1} \mathrm{H}$ NMR $\left(500 \mathrm{MHz}, \mathrm{CDCl}_{3}\right) \delta 2.69$ (bs, $\left.1 \mathrm{H}\right), 1.44-1.17(\mathrm{~m}, 22 \mathrm{H}), 0.90(\mathrm{t}, J=$ $7.0 \mathrm{~Hz}, 3 \mathrm{H}), 0.88(\mathrm{t}, J=7.0 \mathrm{~Hz}, 3 \mathrm{H}) .{ }^{13} \mathrm{C} \mathrm{NMR}\left(125 \mathrm{MHz}, \mathrm{CDCl}_{3}\right) \delta 51.4,38.3,32.2,32.0,30.0$, 29.5, 26.4, 26.0, 22.8, 14.2. $[\alpha]^{24}{ }_{\mathrm{D}}=-23.69\left(c\right.$ 0.5, $\left.\mathrm{CHCl}_{3}\right)$. HRMS (ESI-TOF) m/z: $[\mathrm{M}+\mathrm{H}]^{+}$ Calcd for $\mathrm{C}_{13} \mathrm{H}_{30} \mathrm{~N}^{+}$200.2373; Found 200.2385.

(S)-3,3,3-trifluoro-2-methoxy-2-phenyl-N-((S)-tridecan-6-yl)propanamide (18). $\mathrm{Et}_{3} \mathrm{~N}$ (10.5 $\mu \mathrm{L}, 0.0377 \mathrm{mmol}, 2.5$ equiv) and $(S)$-Mosher's acid chloride (3.0 $\mu \mathrm{L}, 0.0158 \mathrm{mmol}, 1.05$ equiv) were added to a stirred solution of $17\left(0.0030 \mathrm{mg}, 0.015 \mathrm{mmol}, 1\right.$ equiv) in $\mathrm{CH}_{2} \mathrm{Cl}_{2}(0.5$ $\mathrm{mL}$ ), at room temperature. The reaction mixture was adsorbed onto silica gel and purified via flash column chromatography (5\% EtOAc in hexanes) to afford $\mathbf{1 8}(0.0056 \mathrm{~g}, 0.0135 \mathrm{mmol})$ in $90 \%$ yield as a colorless oil. $\mathrm{R}_{f}=0.65\left(20 \%\right.$ EtOAc in hexanes, UV/ $\mathrm{KMnO}_{4}$ stain). ${ }^{1} \mathrm{H} \mathrm{NMR}$ $\left(500 \mathrm{MHz}, \mathrm{CDCl}_{3}\right) \delta 7.58-7.52(\mathrm{~m}, 2 \mathrm{H}), 7.41-7.38(\mathrm{~m}, 3 \mathrm{H}), 6.43(\mathrm{~d}, J=9.3 \mathrm{~Hz}, 1 \mathrm{H}), 4.03-$ $3.87(\mathrm{~m}, 1 \mathrm{H}), 3.44(\mathrm{~s}, 3 \mathrm{H}), 1.61-1.46(\mathrm{~m}, 2 \mathrm{H}), 1.45-1.16(\mathrm{~m}, 18 \mathrm{H}), 0.94-0.81(\mathrm{~m}, 6 \mathrm{H}) .{ }^{19} \mathrm{~F}$ NMR (282 MHz, $\left.\mathrm{CDCl}_{3}\right) \delta-68.79$. HRMS (ESI-TOF) m/z: [M+H $]^{+}$Calcd for $\mathrm{C}_{23} \mathrm{H}_{37} \mathrm{NF}_{3} \mathrm{O}_{2}{ }^{+}$ 416.2771; Found 416.2794. Unfortunately, all the peaks for the two diastereomers of 18 overlap in the ${ }^{1} \mathrm{H}$ and ${ }^{19} \mathrm{~F}$ NMR, thus the diastereomeric ratio was determined by HPLC with a Chiralcel OD-H column (99.75 : $0.25 n$-hexane:isopropanol, $0.2 \mathrm{~mL} / \mathrm{min}, 254 \mathrm{~nm}$ ); minor $\mathrm{t}_{\mathrm{r}}=31.9 \mathrm{~min}$; major $\mathrm{t}_{\mathrm{r}}=32.8 \mathrm{~min}$; ca. $91: 9 \mathrm{dr}$. 
(S)-N-(tridecan-6-yl)acetamide (4). $\mathrm{Et}_{3} \mathrm{~N}(10.5 \mu \mathrm{L}, 0.0377 \mathrm{mmol}, 2.5$ equiv), and acetyl chloride (1.1 $\mu \mathrm{L}, 0.0158 \mathrm{mmol}, 1.05$ equiv) were added to a stirred solution of $\mathbf{1 7}(0.0030 \mathrm{mg}$, 0.015 mmol, 1 equiv) in $\mathrm{CH}_{2} \mathrm{Cl}_{2}(0.5 \mathrm{~mL})$, at room temperature. After complete reaction, the mixture was adsorbed onto silica gel and flash chromatography (5\% EtOAc in hexanes) afforded $4(0.0032 \mathrm{~g}, 0.0132 \mathrm{mmol})$ in $88 \%$ yield as white solid. NMR data for this compound matched previously reported data for the isolated natural sample. ${ }^{8} \mathrm{R}_{f}=0.15(20 \%$ EtOAc in hexanes, UV/ $\mathrm{KMnO}_{4}$ stain). ${ }^{1} \mathrm{H}$ NMR $\left(500 \mathrm{MHz}, \mathrm{CDCl}_{3}\right) \delta 5.13(\mathrm{~d}, J=9.3 \mathrm{~Hz}, 1 \mathrm{H}), 3.88(\mathrm{bs}, 1 \mathrm{H}), 1.96(\mathrm{~s}$, $3 \mathrm{H}), 1.51-1.40(\mathrm{~m}, 2 \mathrm{H}), 1.29-1.20(\mathrm{~m}, 18 \mathrm{H}), 0.86(\mathrm{t}, J=6.6 \mathrm{~Hz}, 6 \mathrm{H}) .{ }^{13} \mathrm{C}$ NMR $(125 \mathrm{MHz}$ $\left.\mathrm{CDCl}_{3}\right) \delta 169.7,77.5,77.2,77.0,49.6,35.5,35.4,32.0,29.8,29.5,26.0,25.7,23.8,22.9,22.8$, 14.3, 14.2. $[\alpha]^{24}=+11.17\left(c 0.1, \mathrm{CHCl}_{3}\right)$. The optical rotation of the natural sample was not reported; however, it should be noted that similar isolated natural products (chlorinated derivatives and taveuniamides) have rotation. ${ }^{8,9} \mathrm{MP}=64{ }^{\circ} \mathrm{C}$. HRMS (ESI-TOF) $\mathrm{m} / \mathrm{z}:[\mathrm{M}+\mathrm{H}]^{+}$ Calcd for $\mathrm{C}_{15} \mathrm{H}_{32} \mathrm{NO}^{+}$242.2478; Found 242.2485.

Chromatograms of Mosher's amide 18 (50:50 and $91: 9$ mixture of diastereomers):
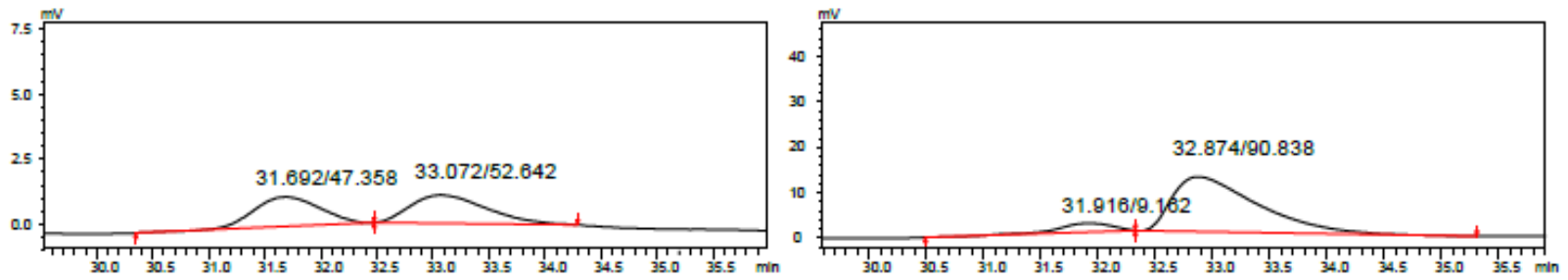


\section{Preliminary Mechanistic Studies}

To gain a better understanding of reaction progress, the formation of $\mathbf{r a c}-9 \mathrm{e}$ by ${ }^{1} \mathrm{H}$ NMR was followed using $\mathrm{CH}_{2} \mathrm{Br}_{2}$ as an internal standard. Experimentally, reactions were run according to the general procedure and quenched by passing aloiquots over a plug of silica at the indicated times. Surprisingly, reaction reached full conversion in $90 \mathrm{~min}$, showing an induction period of approximately 50 minutes (Graph A). Furthermore, a slightly positive non-liner effect ${ }^{10,11}$ was observed for the formation of $9 \mathbf{a}$. At this moment, we are working to understand the factors contributing to the observed induction period and the non-linear effect. These results will be reported in due course.
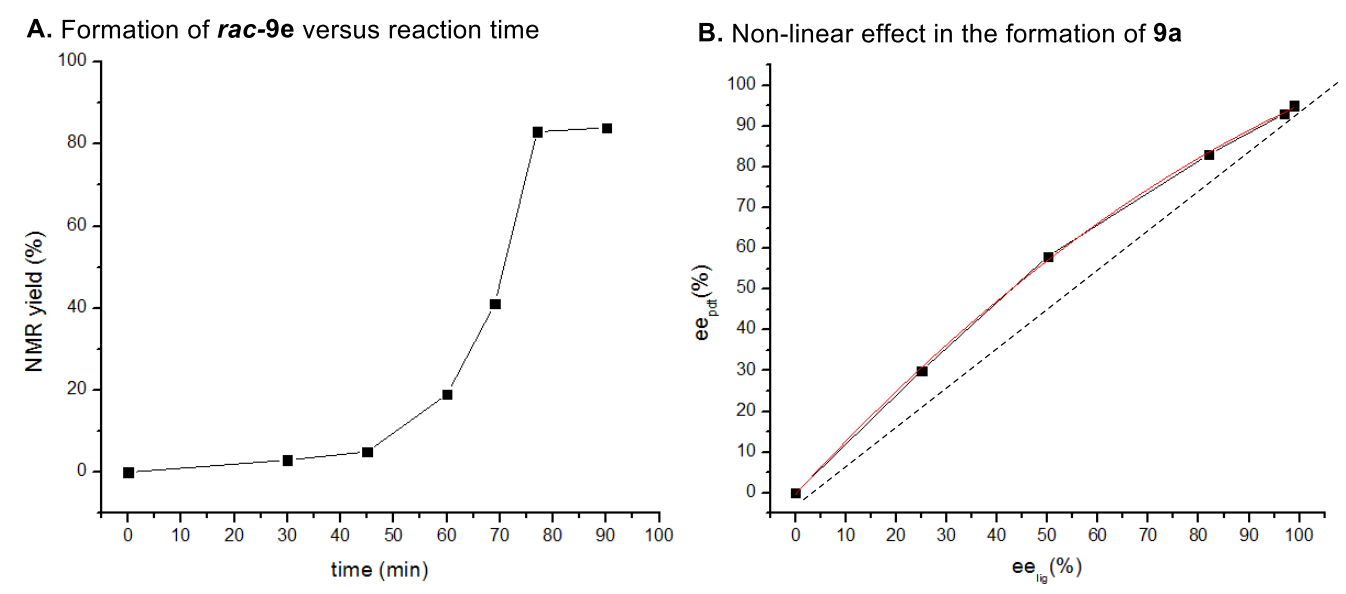

\section{References}

1) Belot, S.; Vogt, K. A.; Besnard, C.; Krause, N.; Alexakis, A. Angew. Chem., Int. Ed. 2009, 48, 8923.

2) Robles, O.; McDonald, F. E. Org. Lett. 2008, 10, 1811.

3) Romanov-Michailidis, F.; Besnard, C.; Alexakis, A. Org. Lett. 2012, 14, 4906.

4) Tretyakov, E. V.; Tkachev, A. V.; Rybalova, T. V.; Gatilov, Y. V.; Knight, D. W.; Vasilevsky, S. F. Tetrahedron 2000, 56, 10075.

5) Mori, M.; Chiba, K.; Okita, M.; Kayo, I.; Ban, Y. Tetrahedron 1985, 41, 375. 
6) Hatano, M.; Mikami, K. J. Am. Chem. Soc. 2003, 125, 4704.

7) Koradin, C.; Polborn, K.; Knochel, P. Angew. Chem., Int. Ed. 2002, 41, 2535.

8) Orsini, M. A.; Pannell, L. K.; Erickson, K. L. J. Nat. Prod. 2001, 64, 572.

9) Williamson, R. T.; Singh, I. P.; Gerwick, W. H. Tetrahedron 2004, 60, 7025.

10) Girard, C.; Kagan, H. B. Angew. Chem., Int. Ed. 1998, 37, 2922.

11) Gommermann, N.; Knochel, P. Chem. Eur. J. 2006, 12, 4380. 
NMR Spectra of Compounds 
7. NMR Spectra of Aldehydes, Alkynes and Amine Starting Materials 


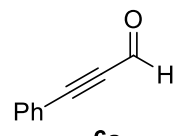

$6 a$
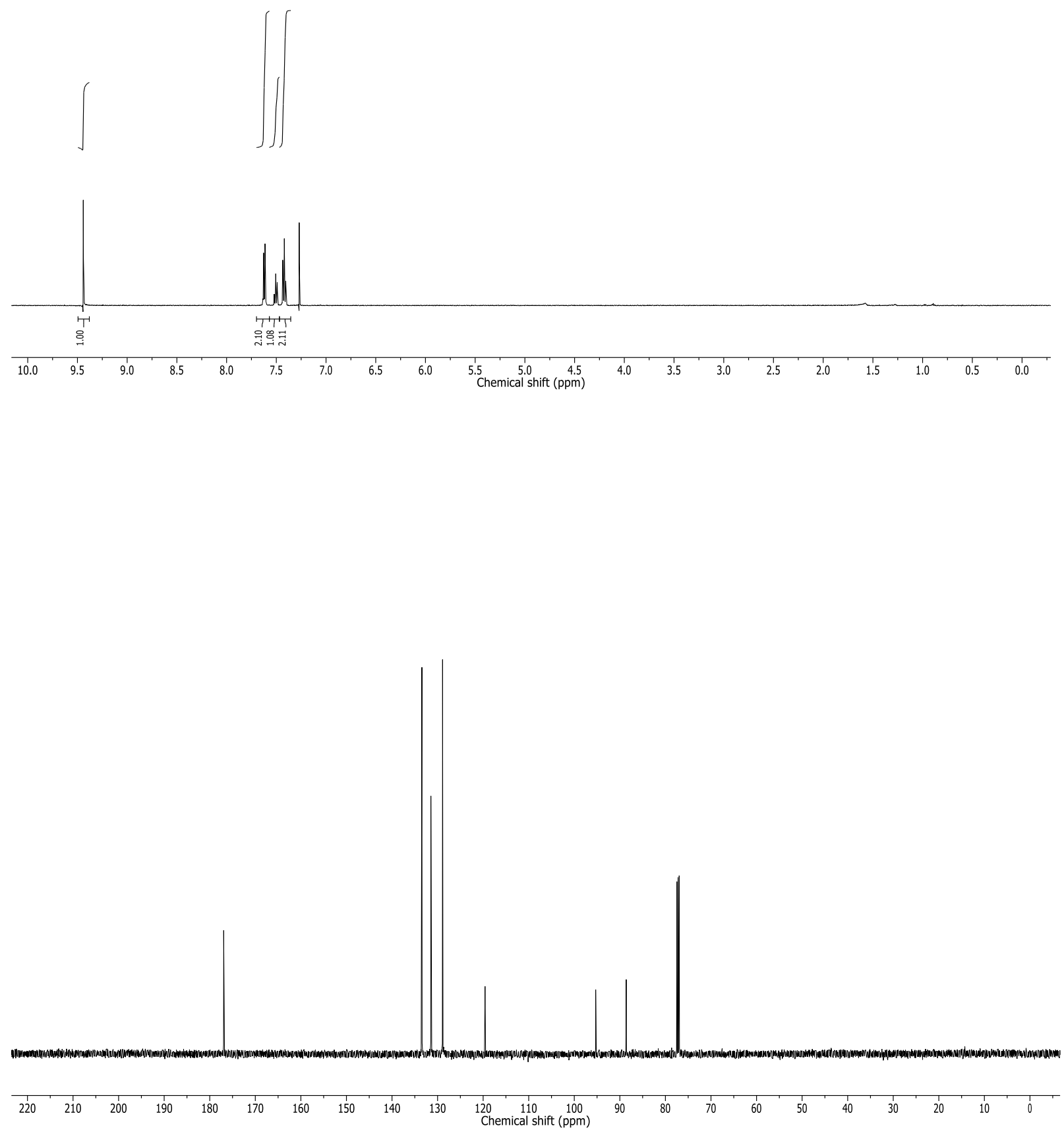

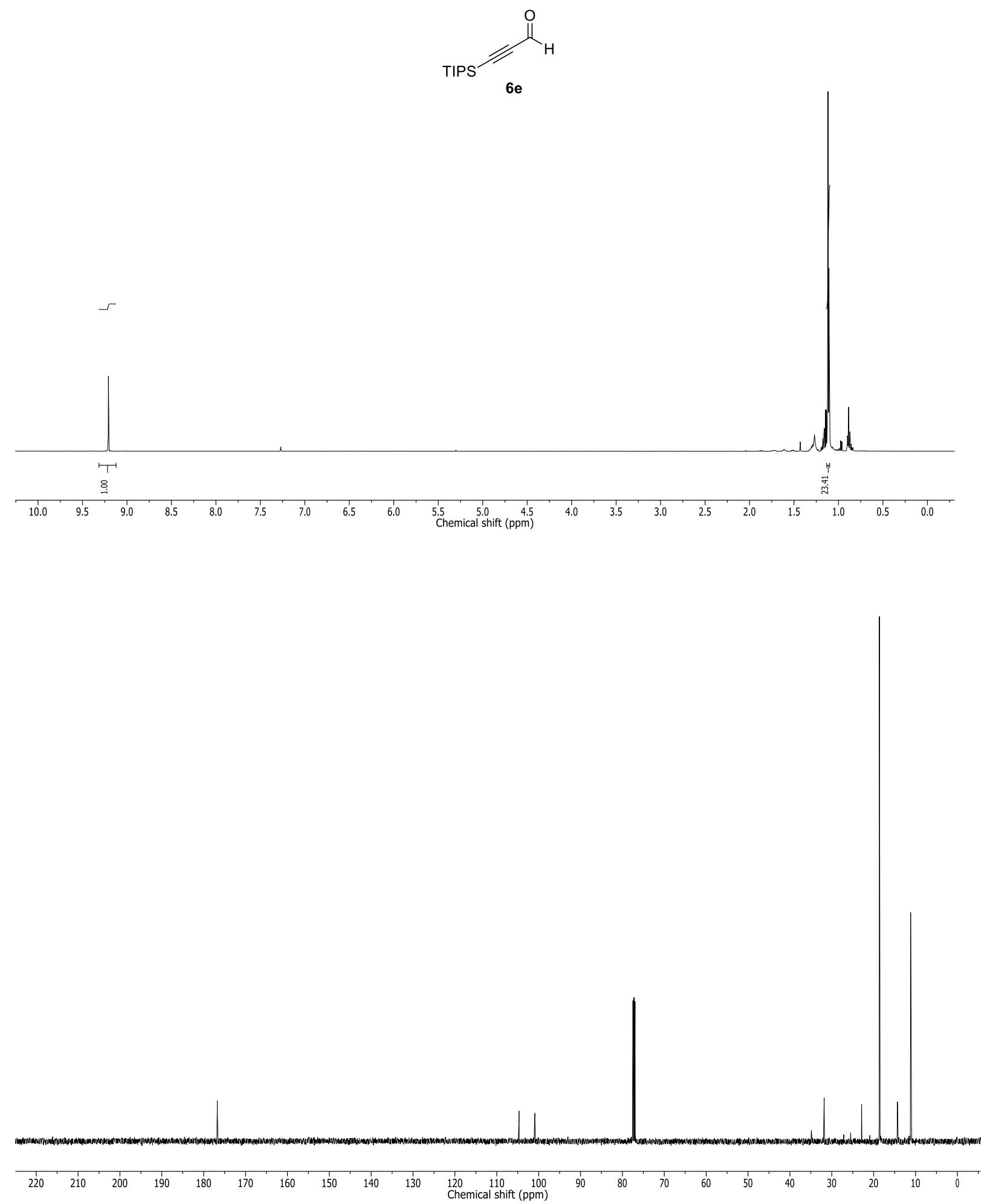

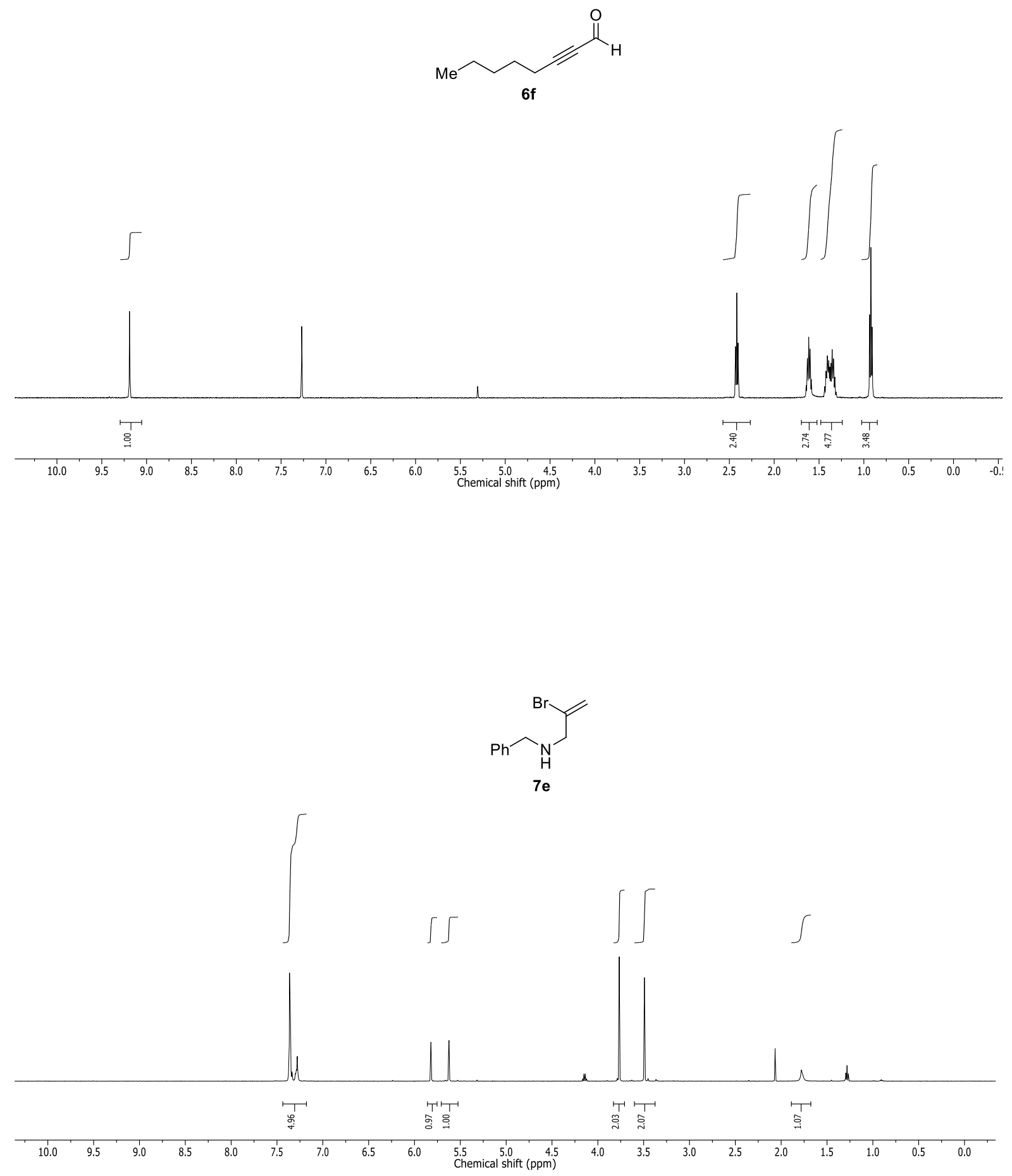

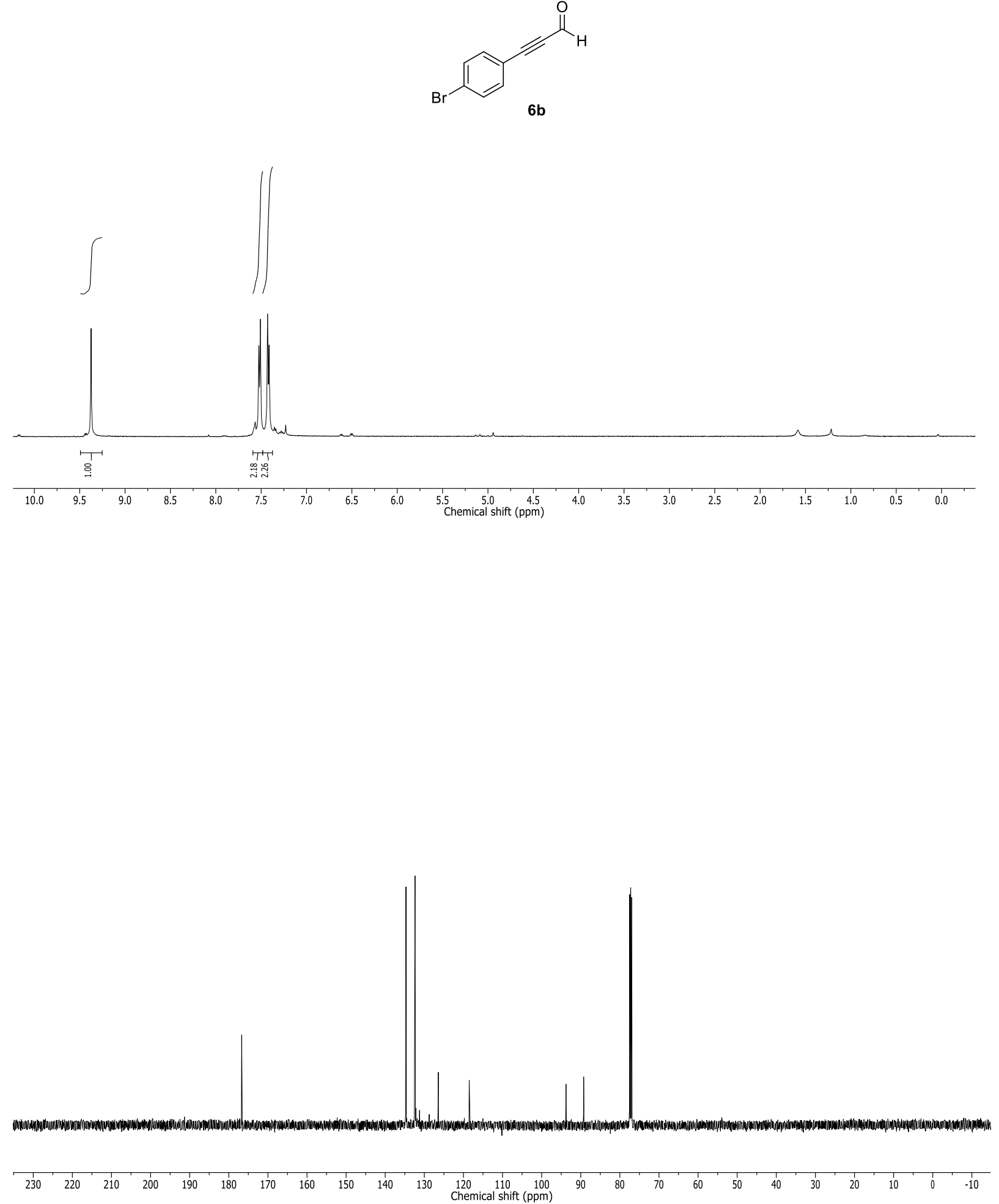

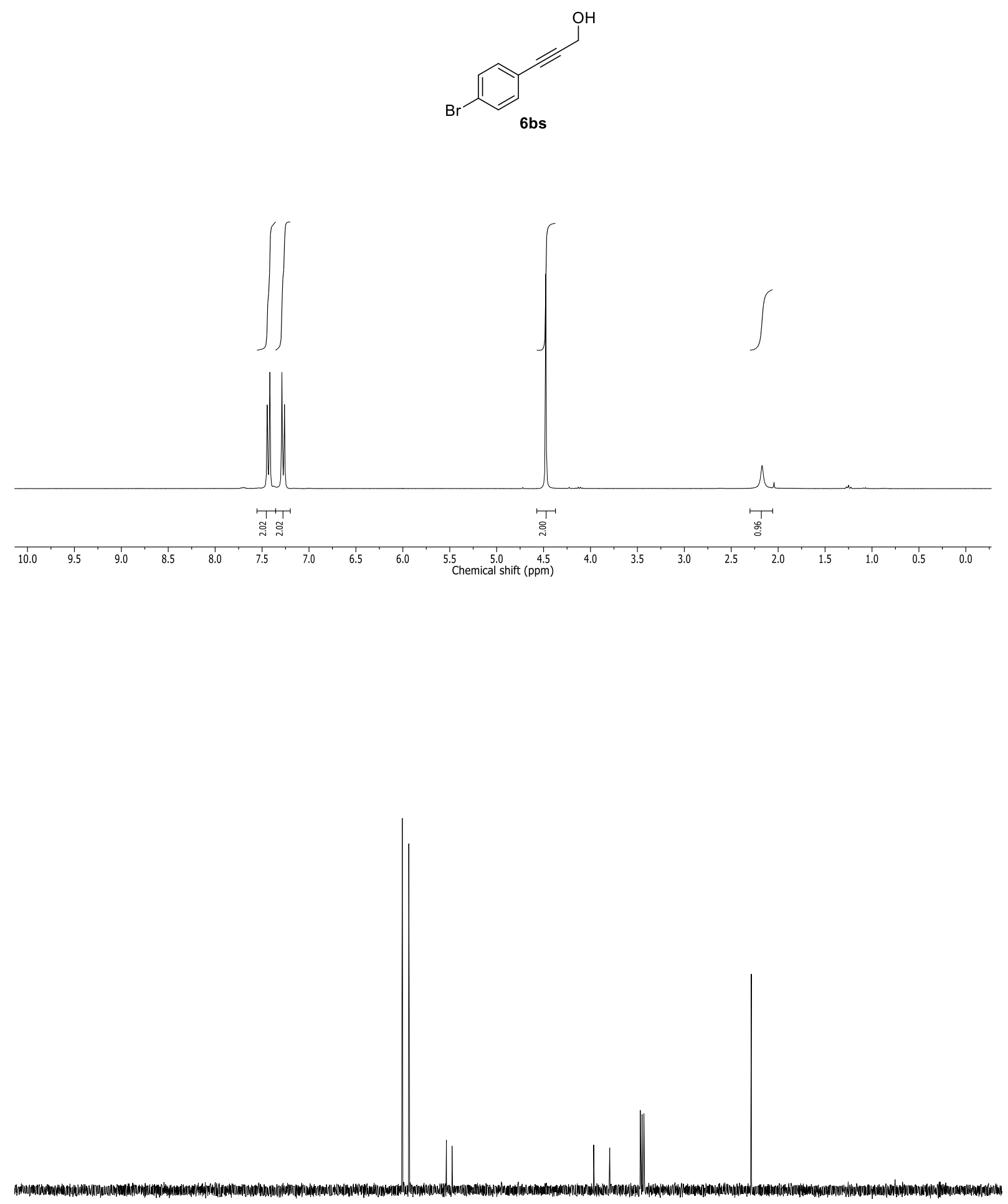

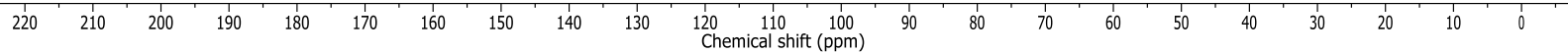



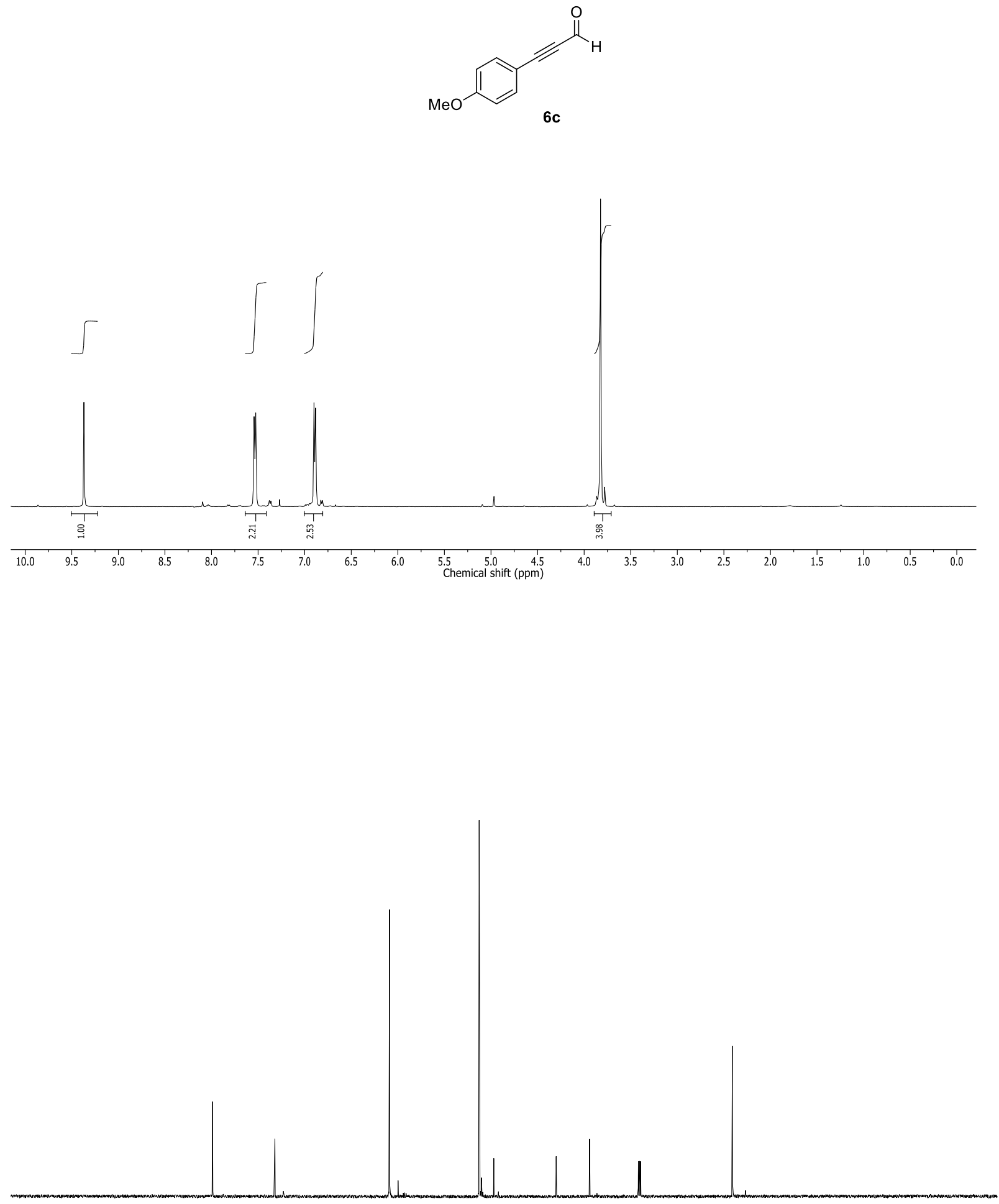

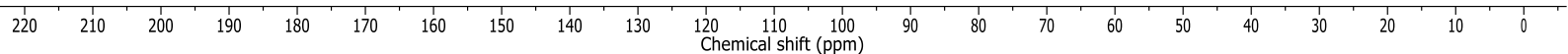



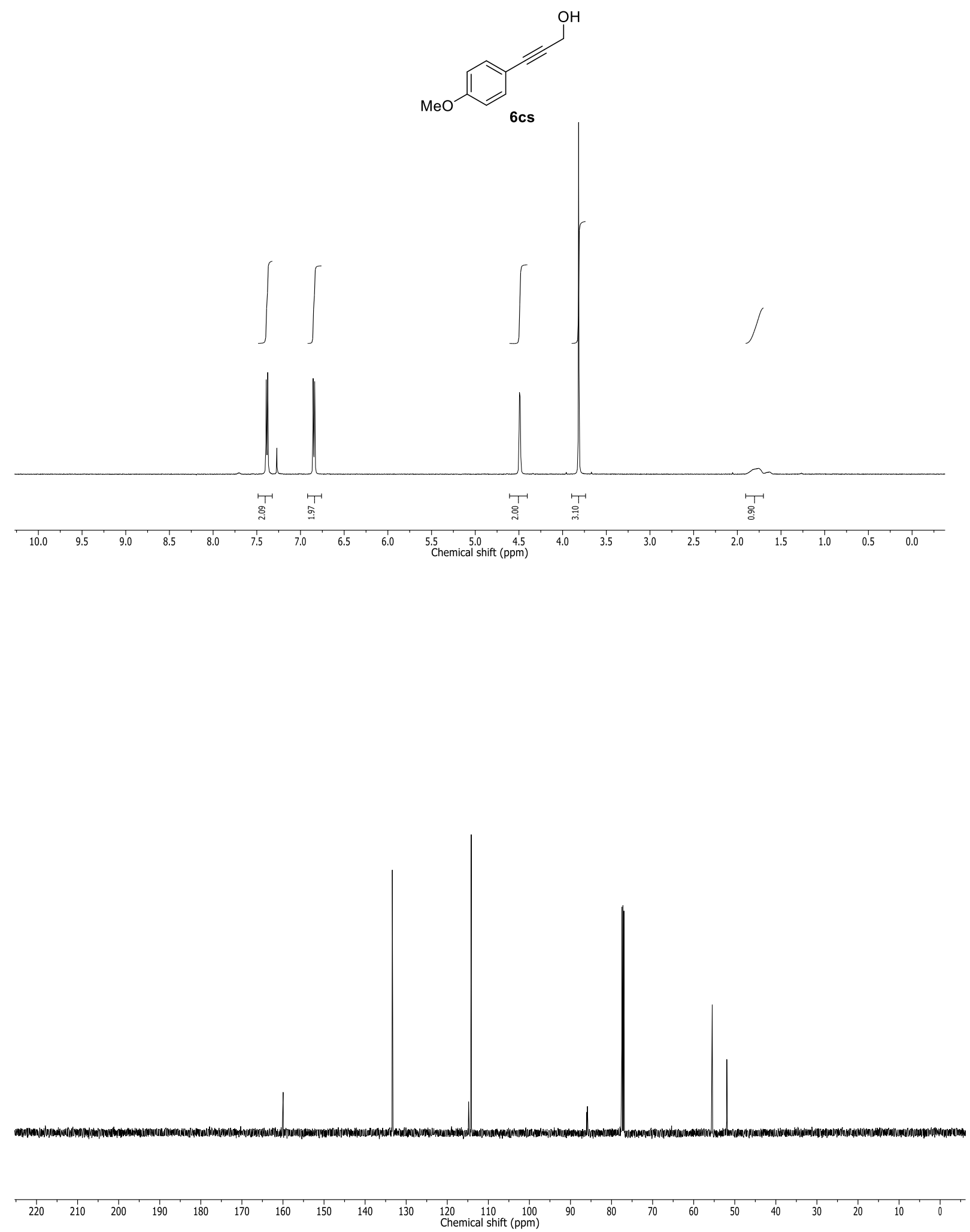


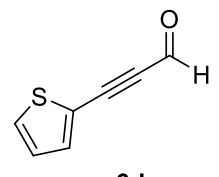

6d
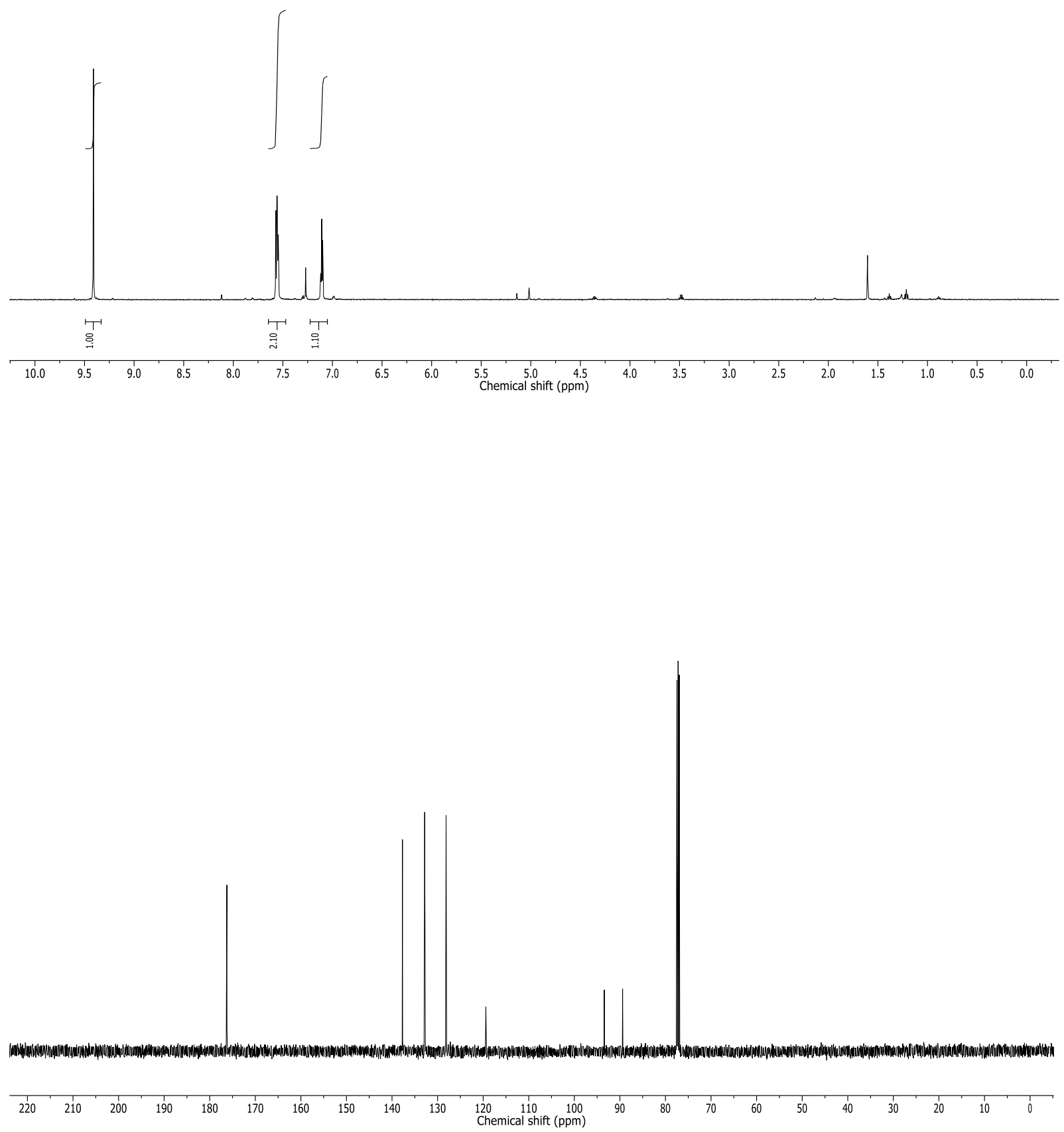

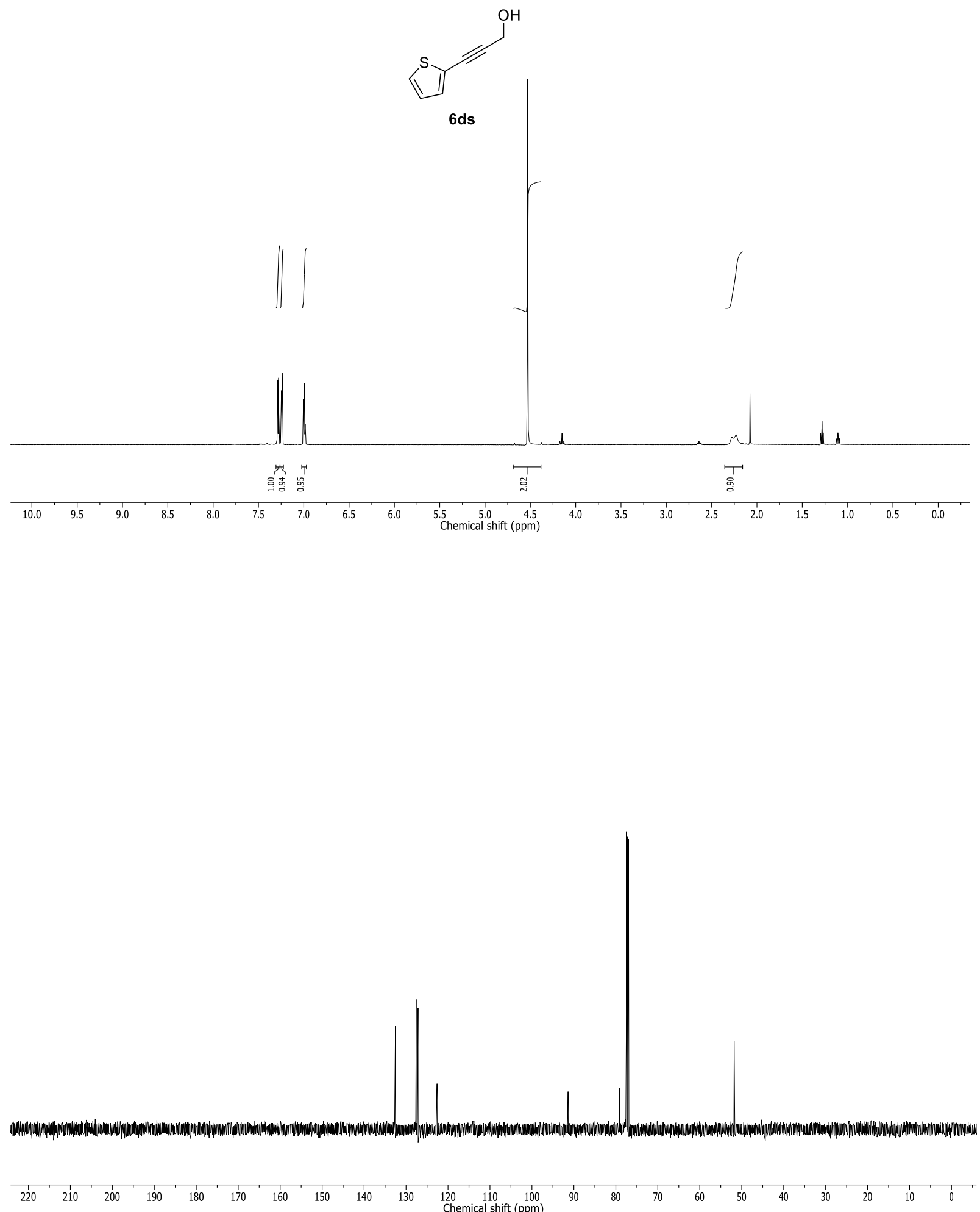


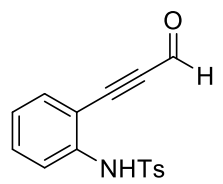

69
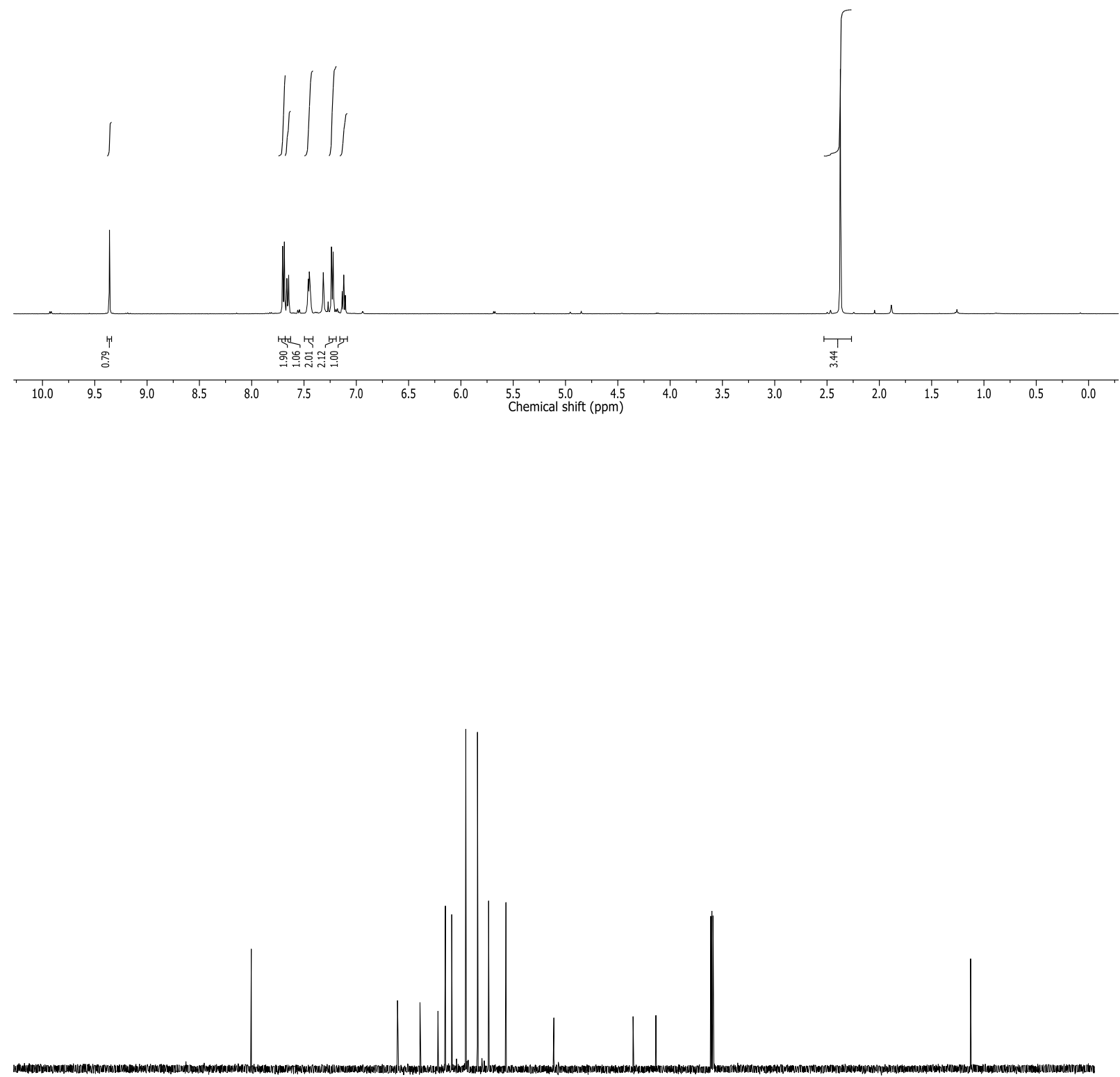

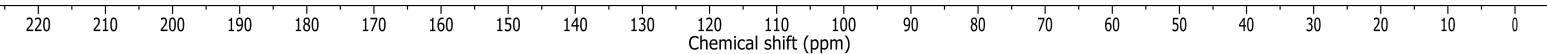




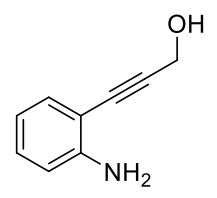

6gs
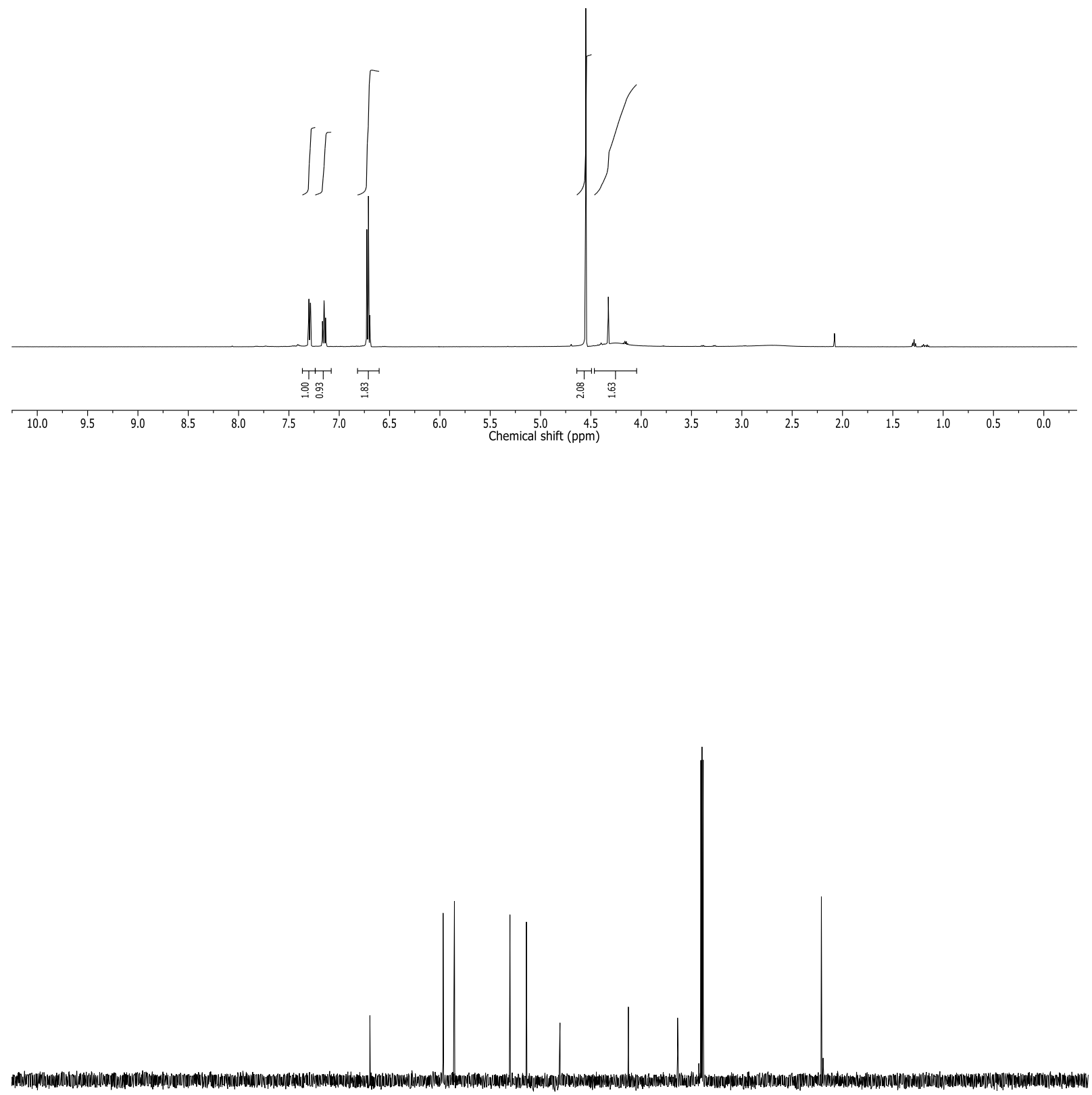

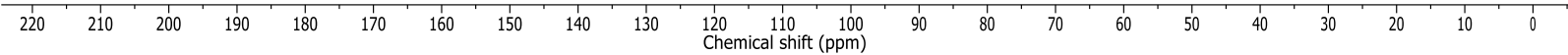



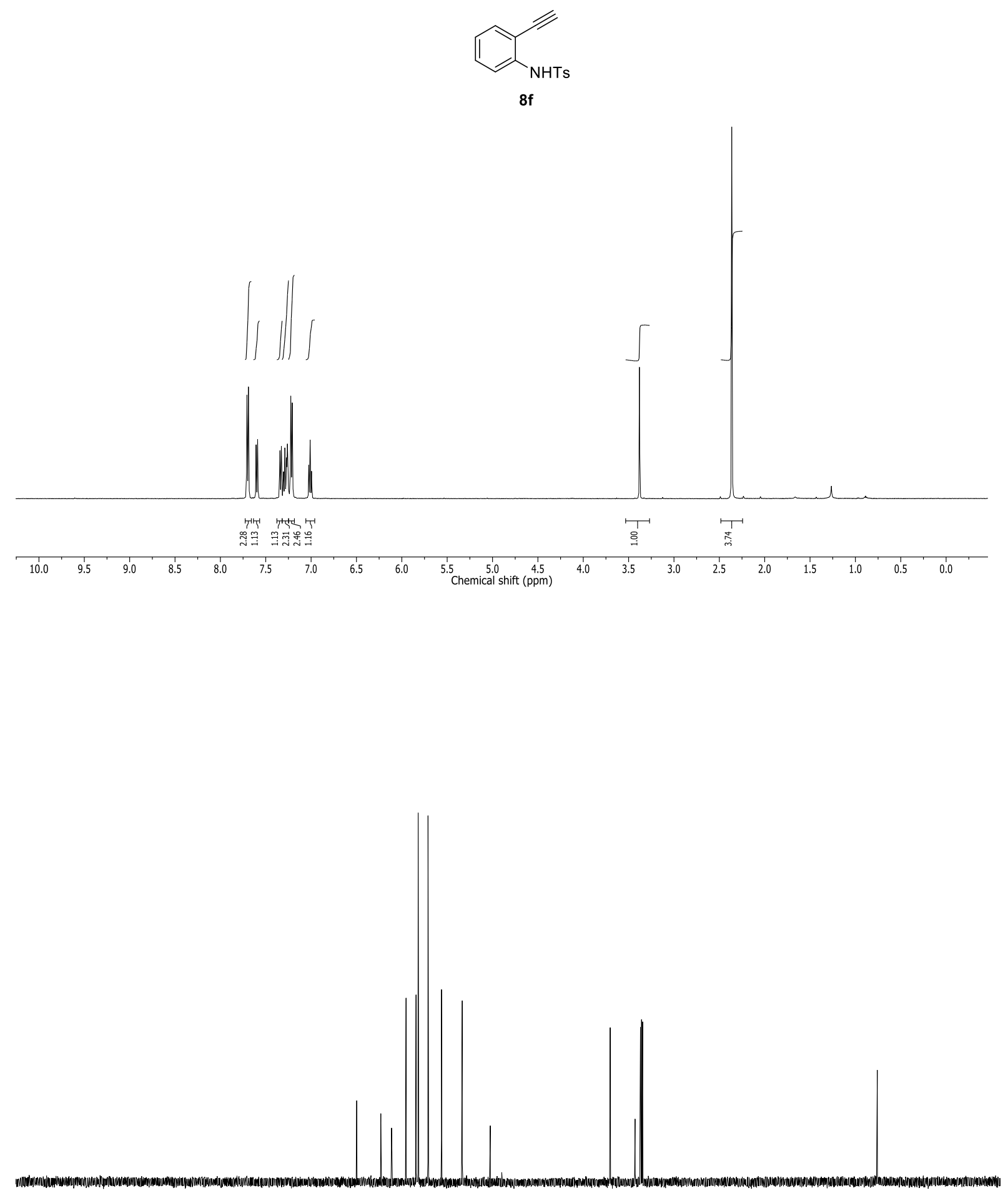

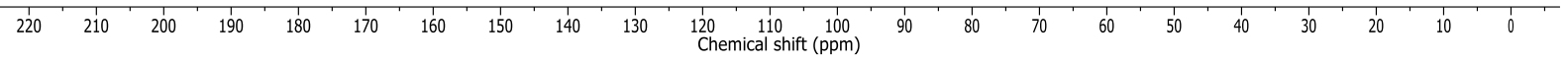


8. NMR Spectra of 3-amino-1,4-diynes 9a-q 

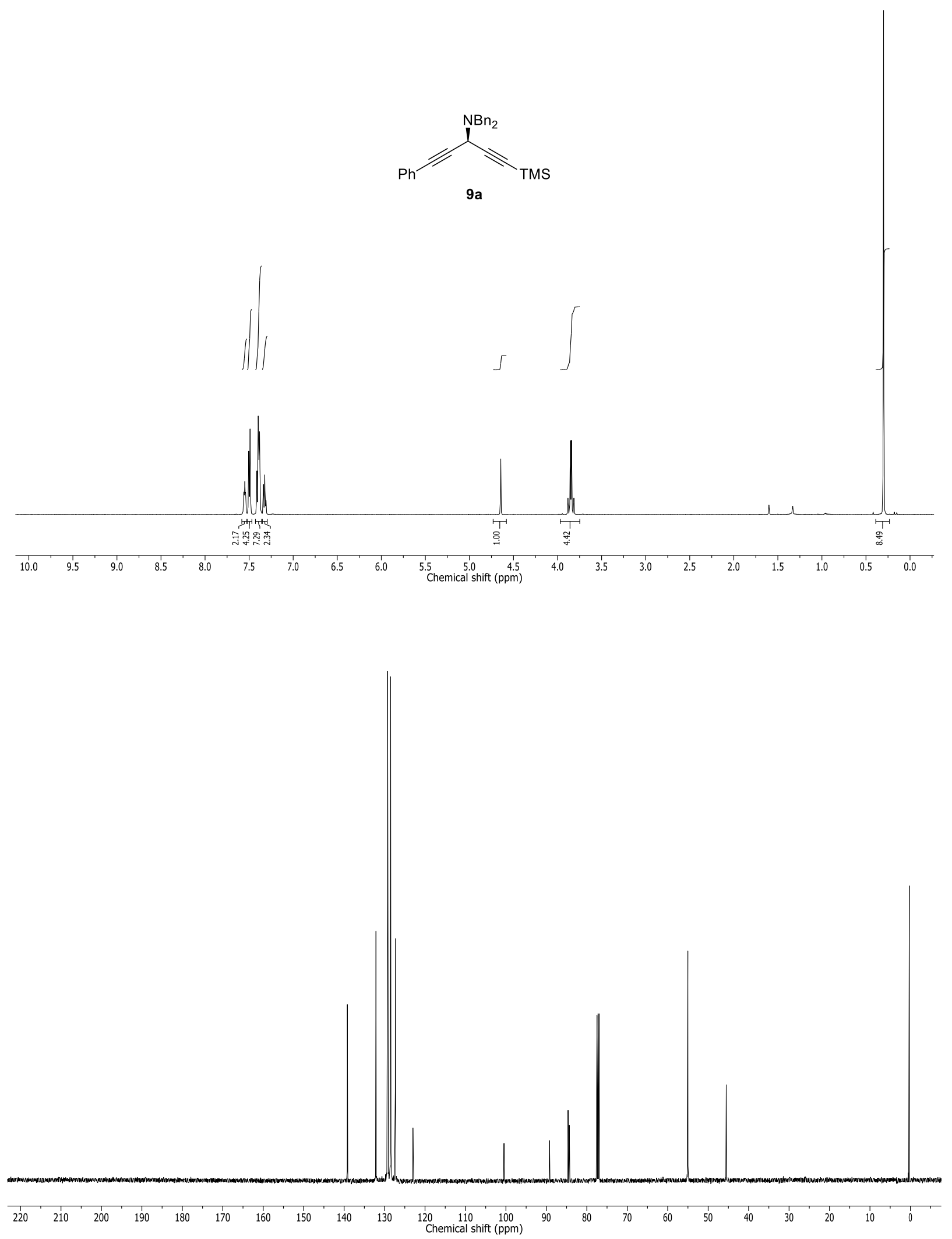

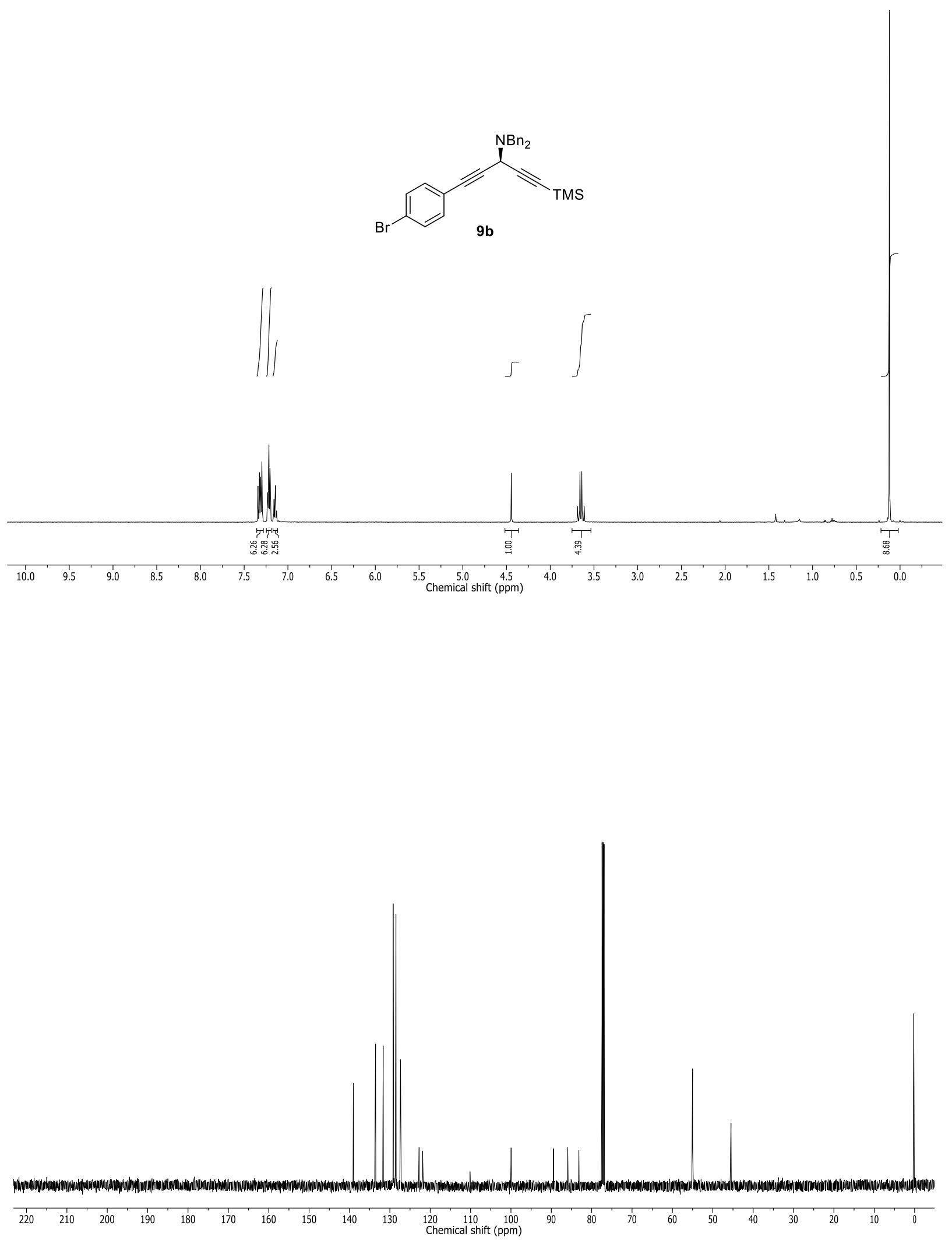

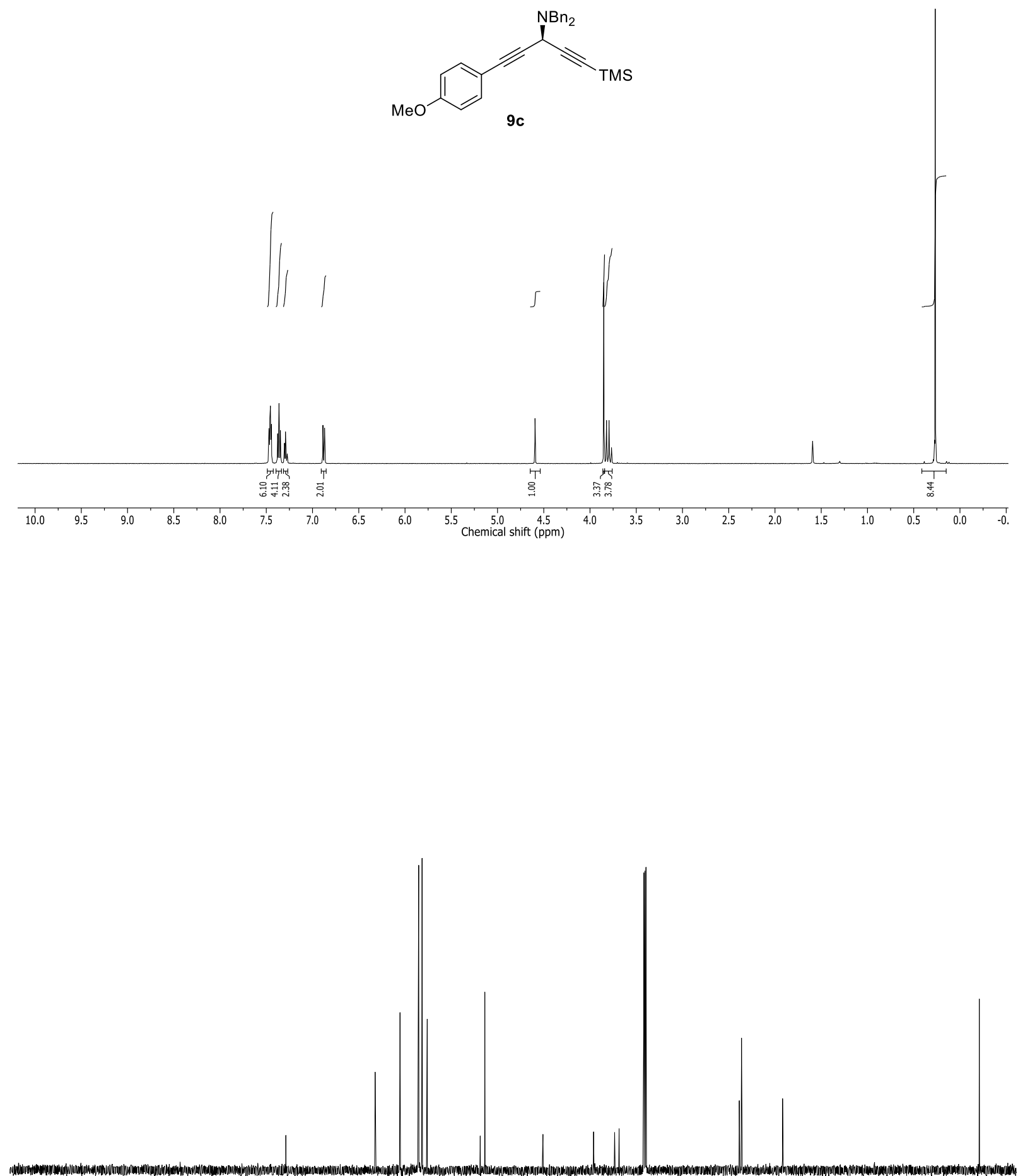

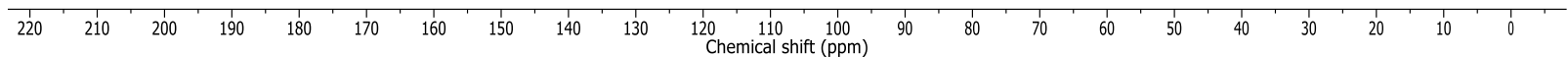



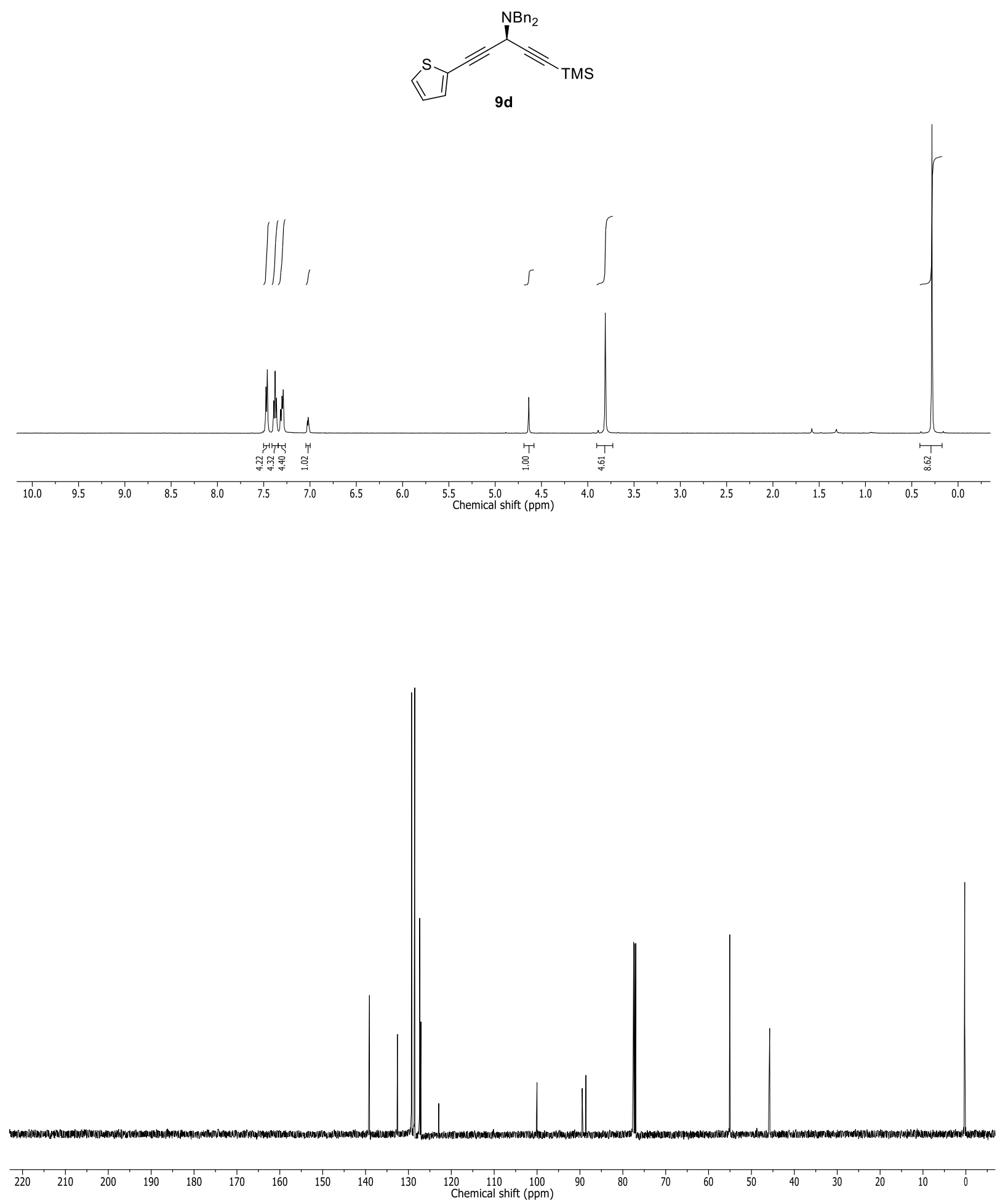

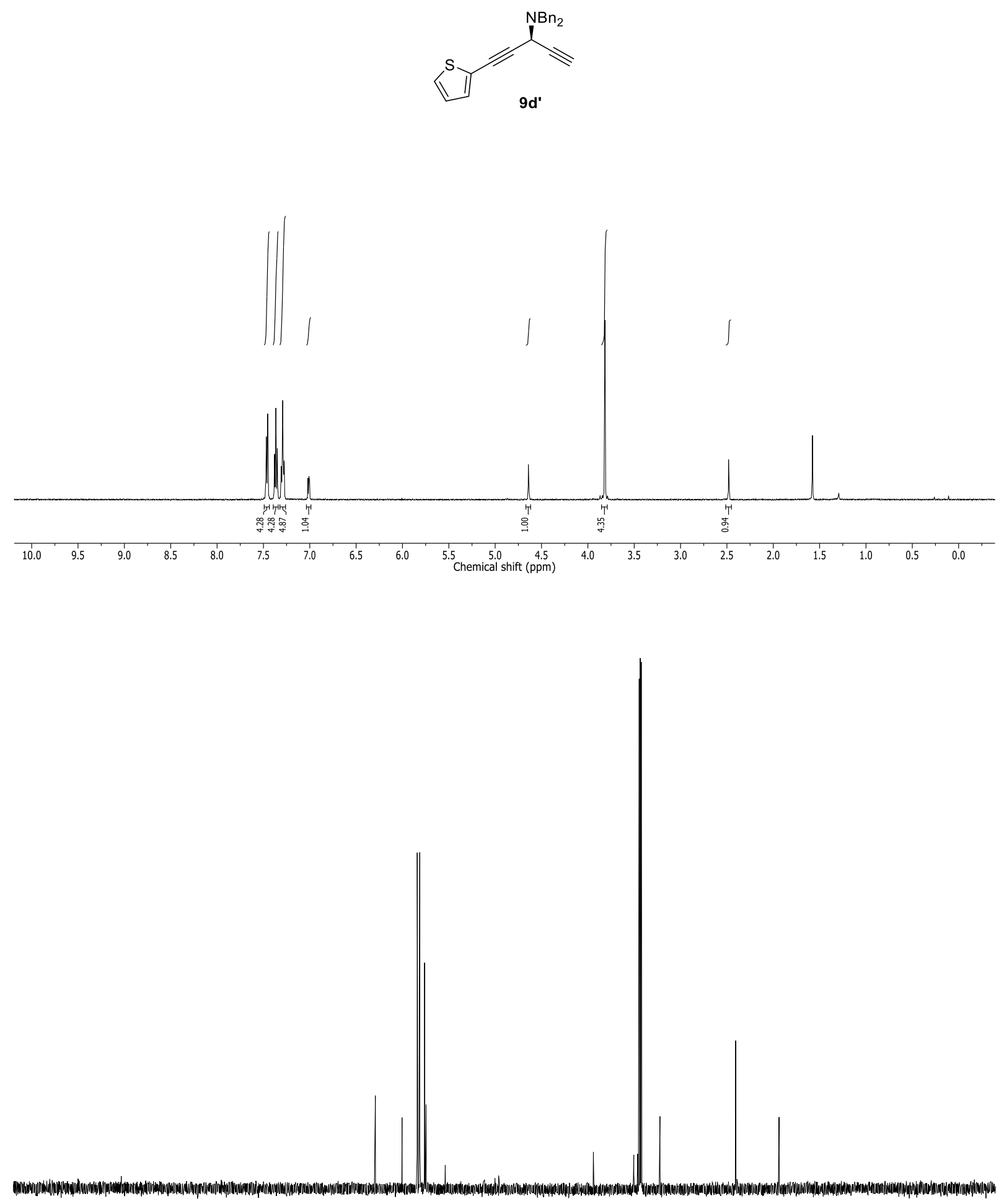

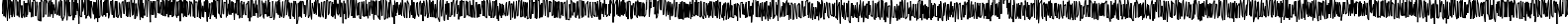

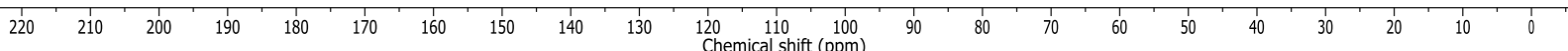



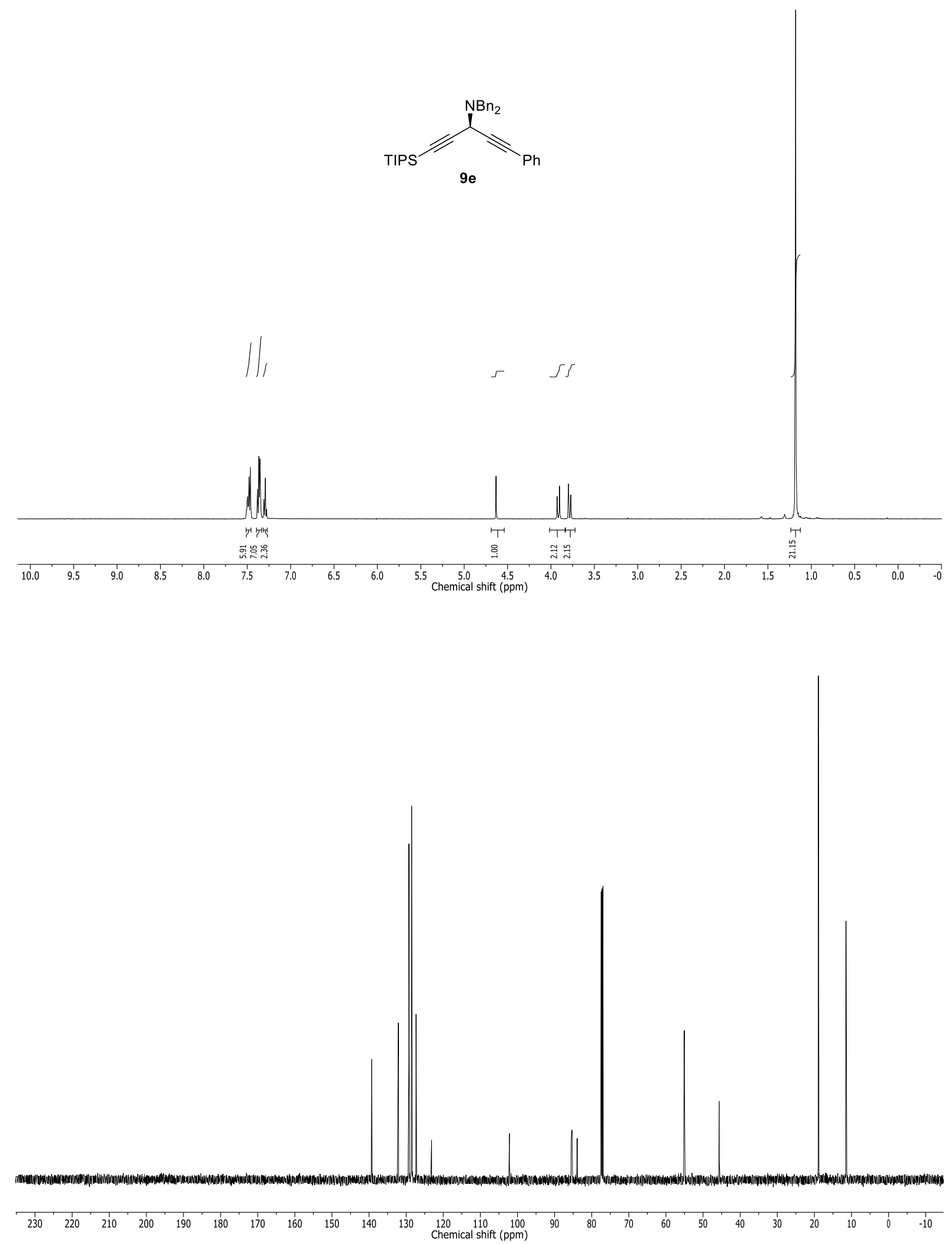

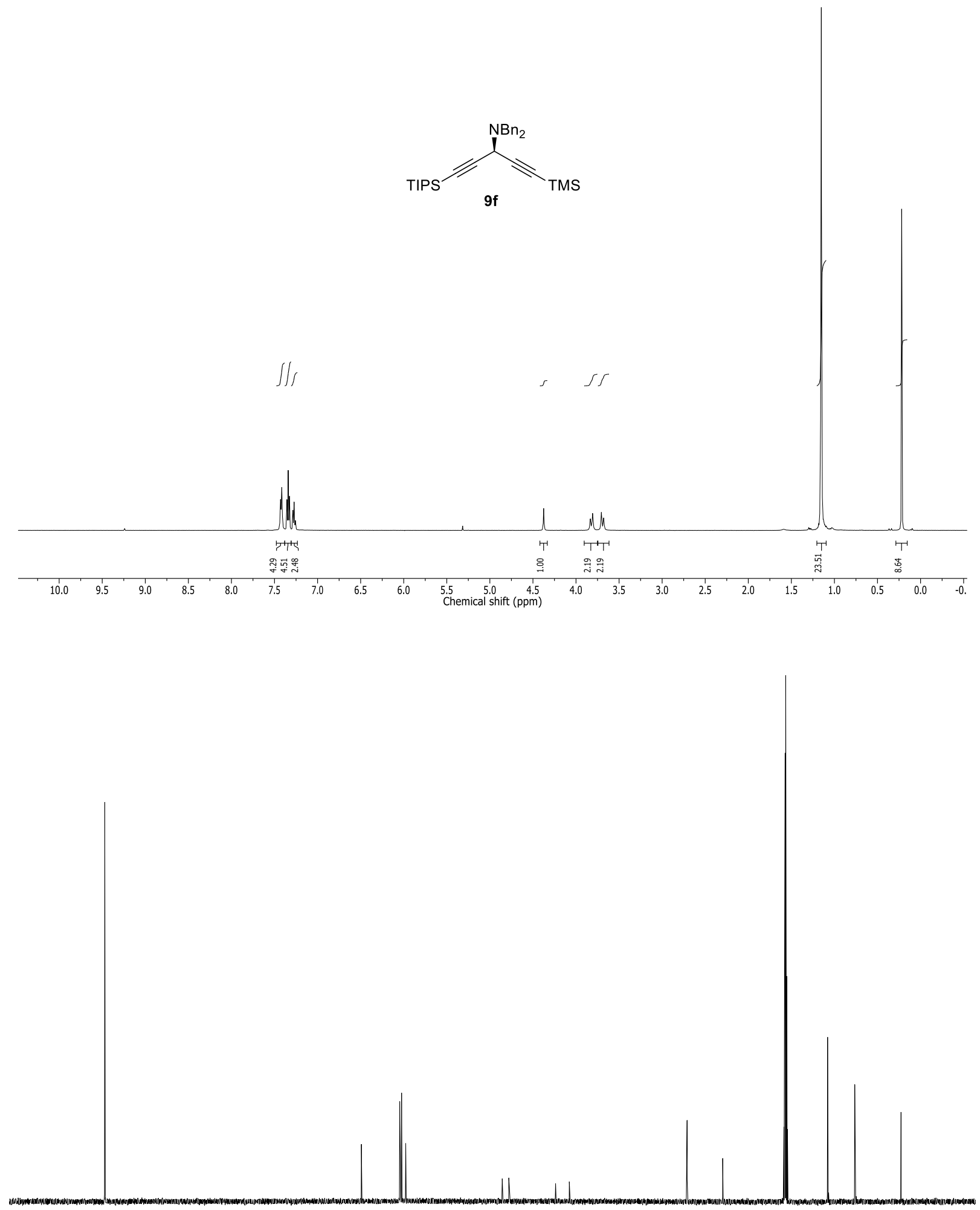

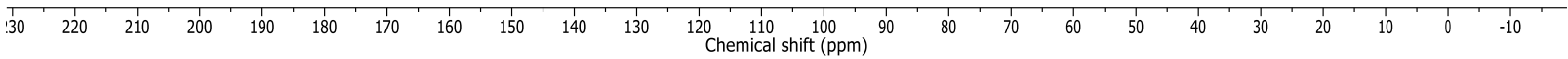



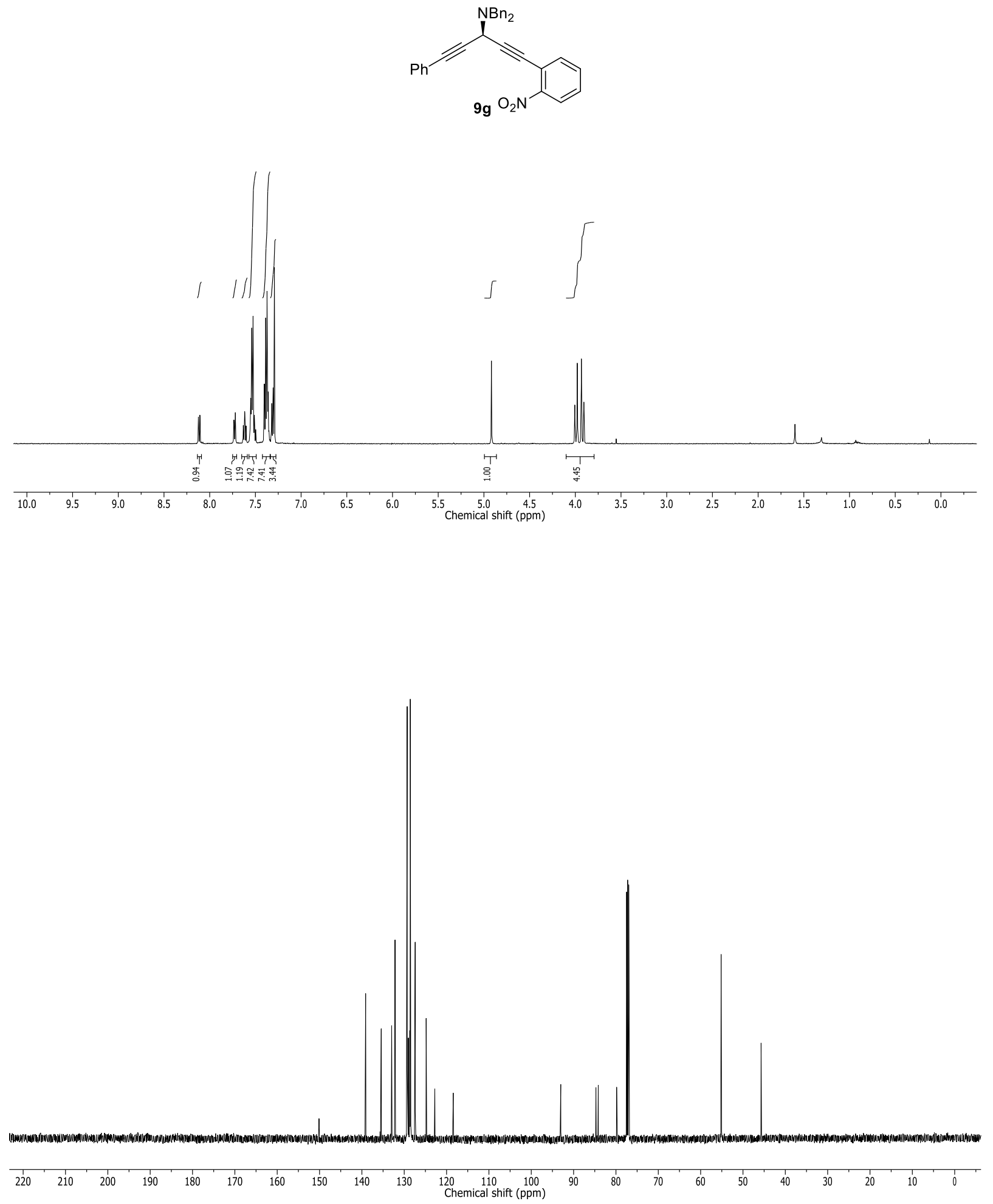

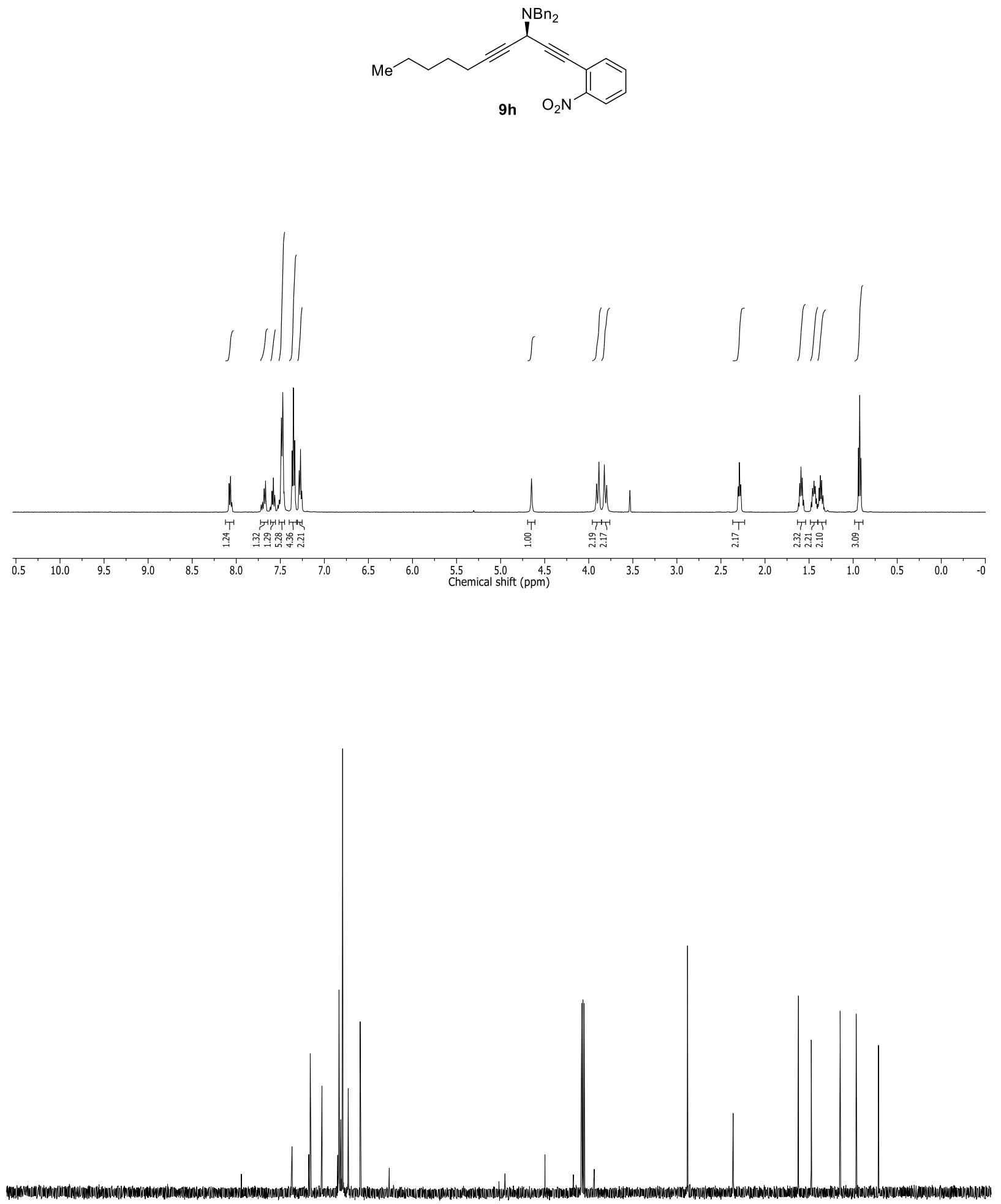

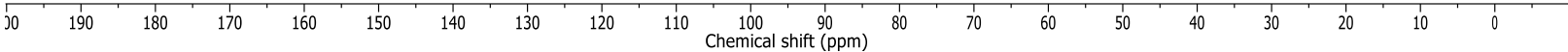



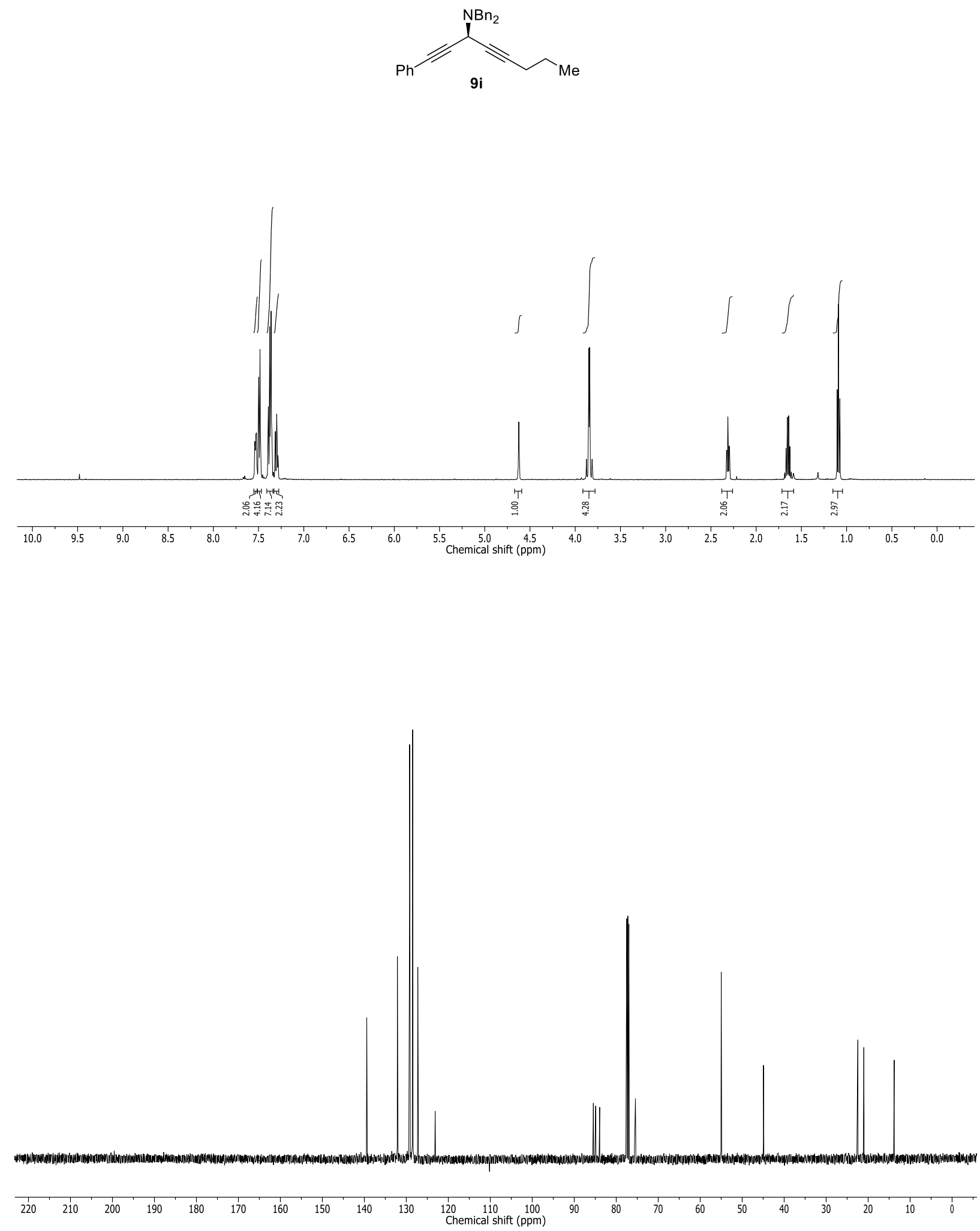

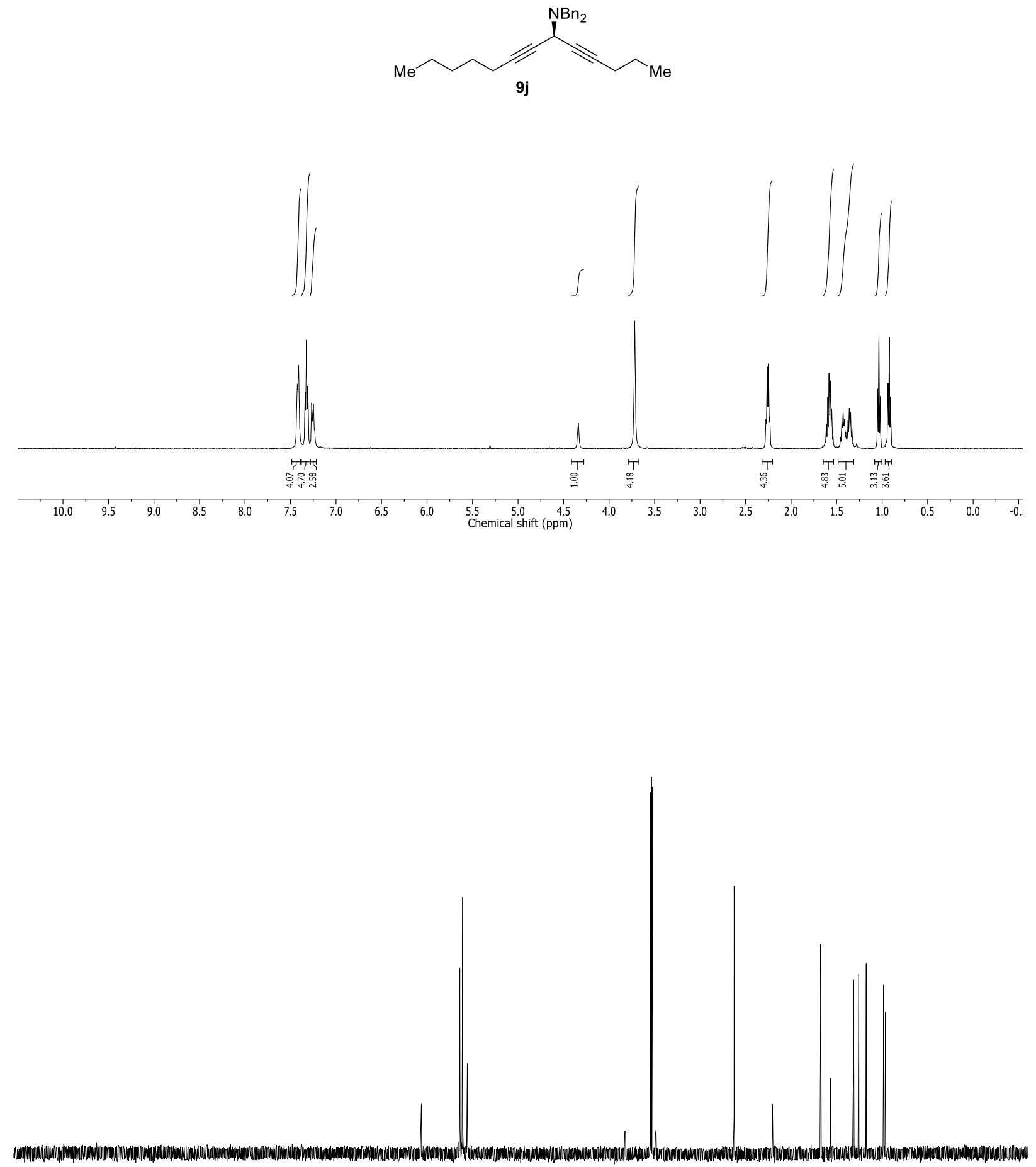

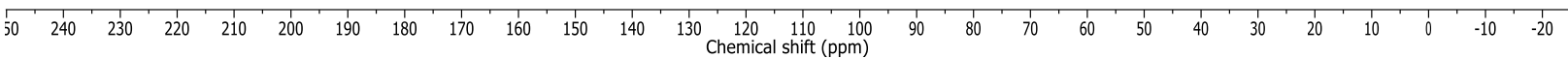



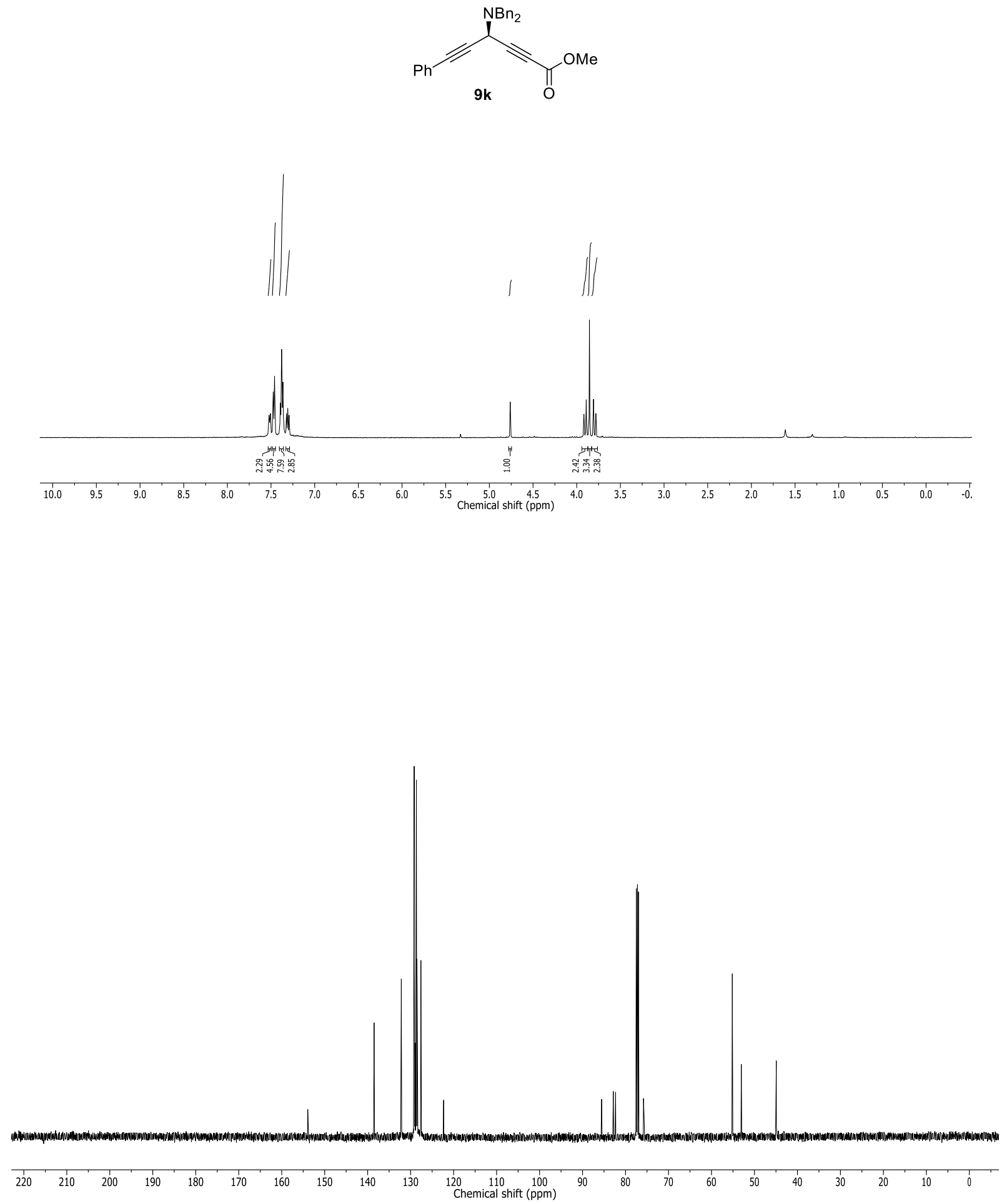

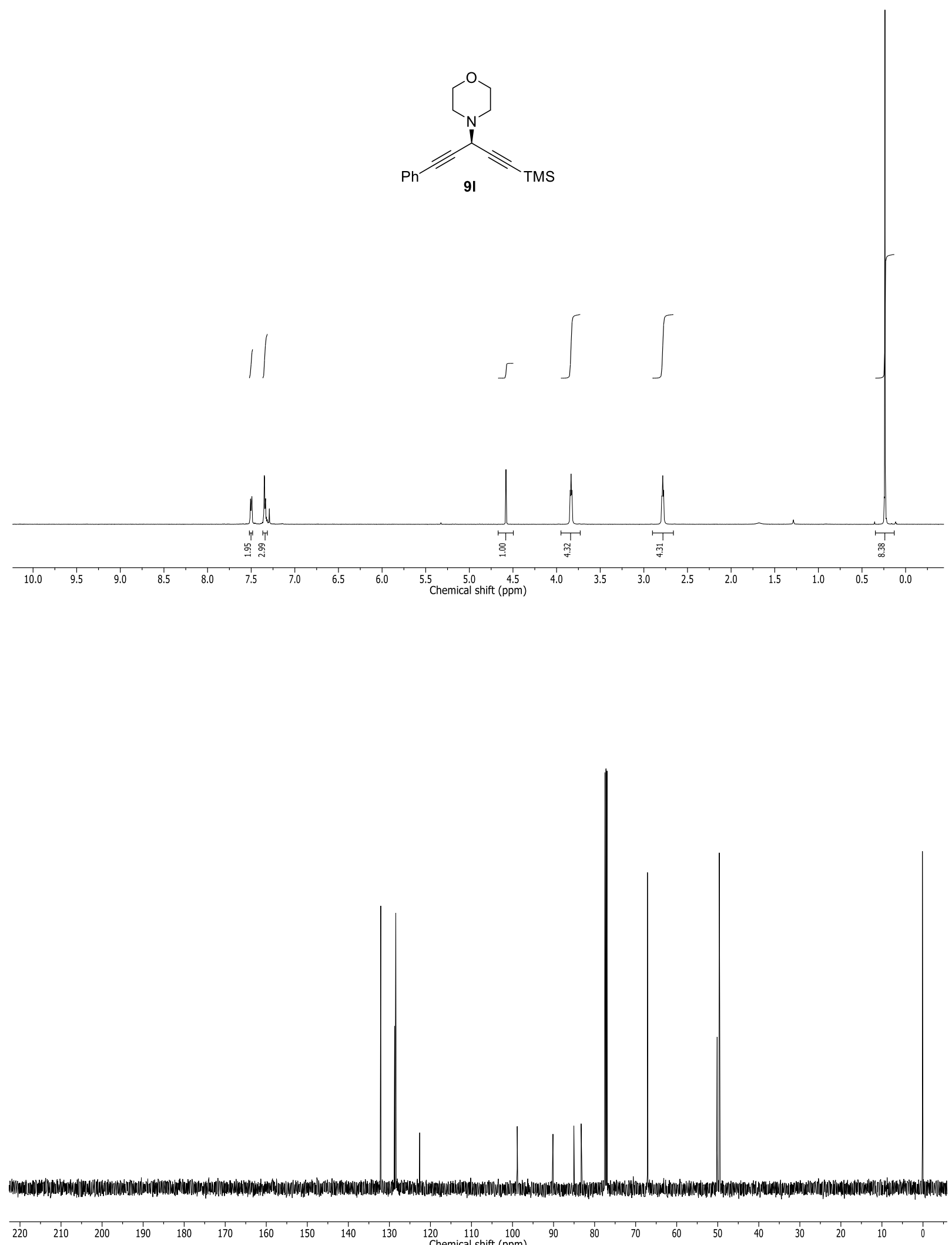

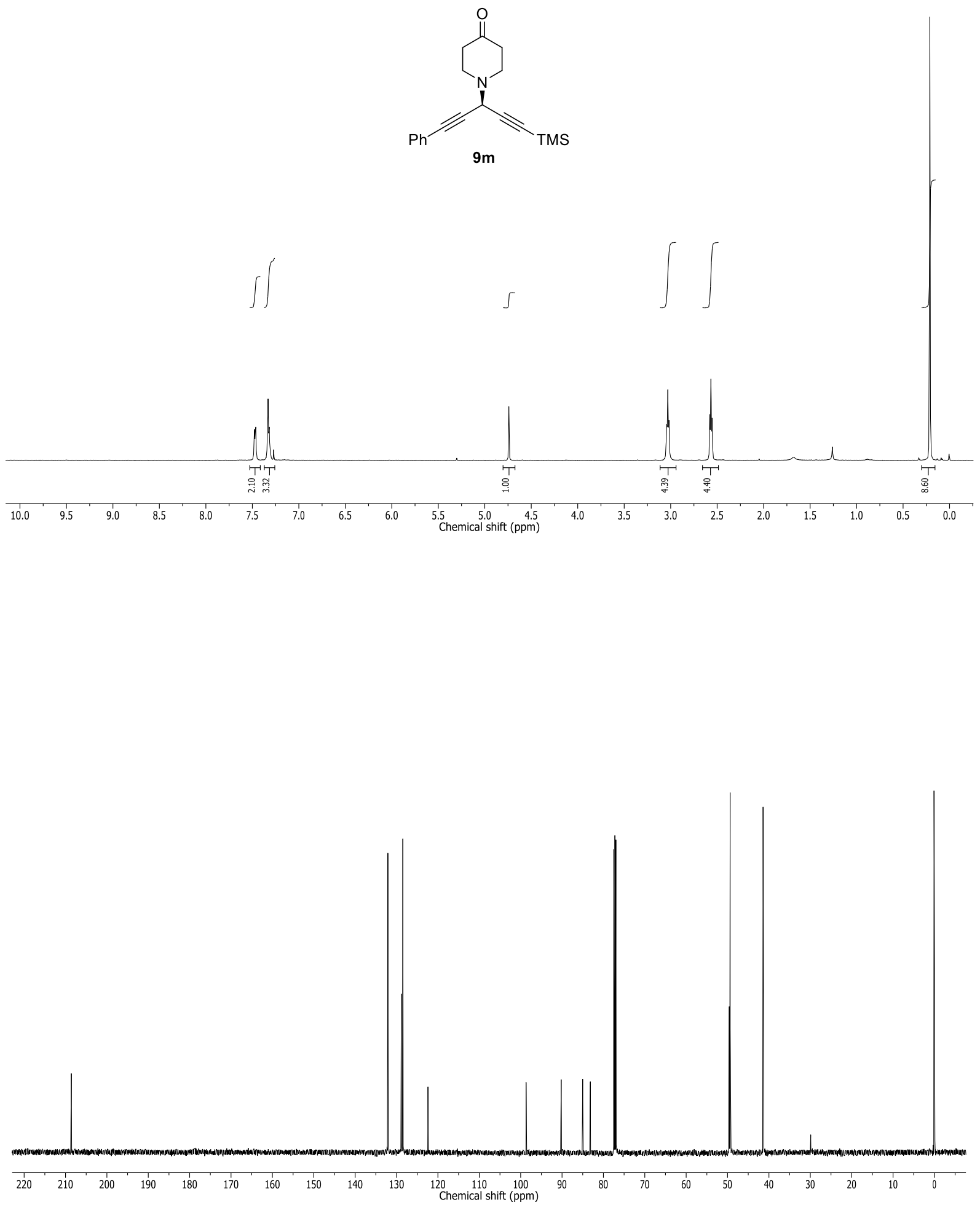

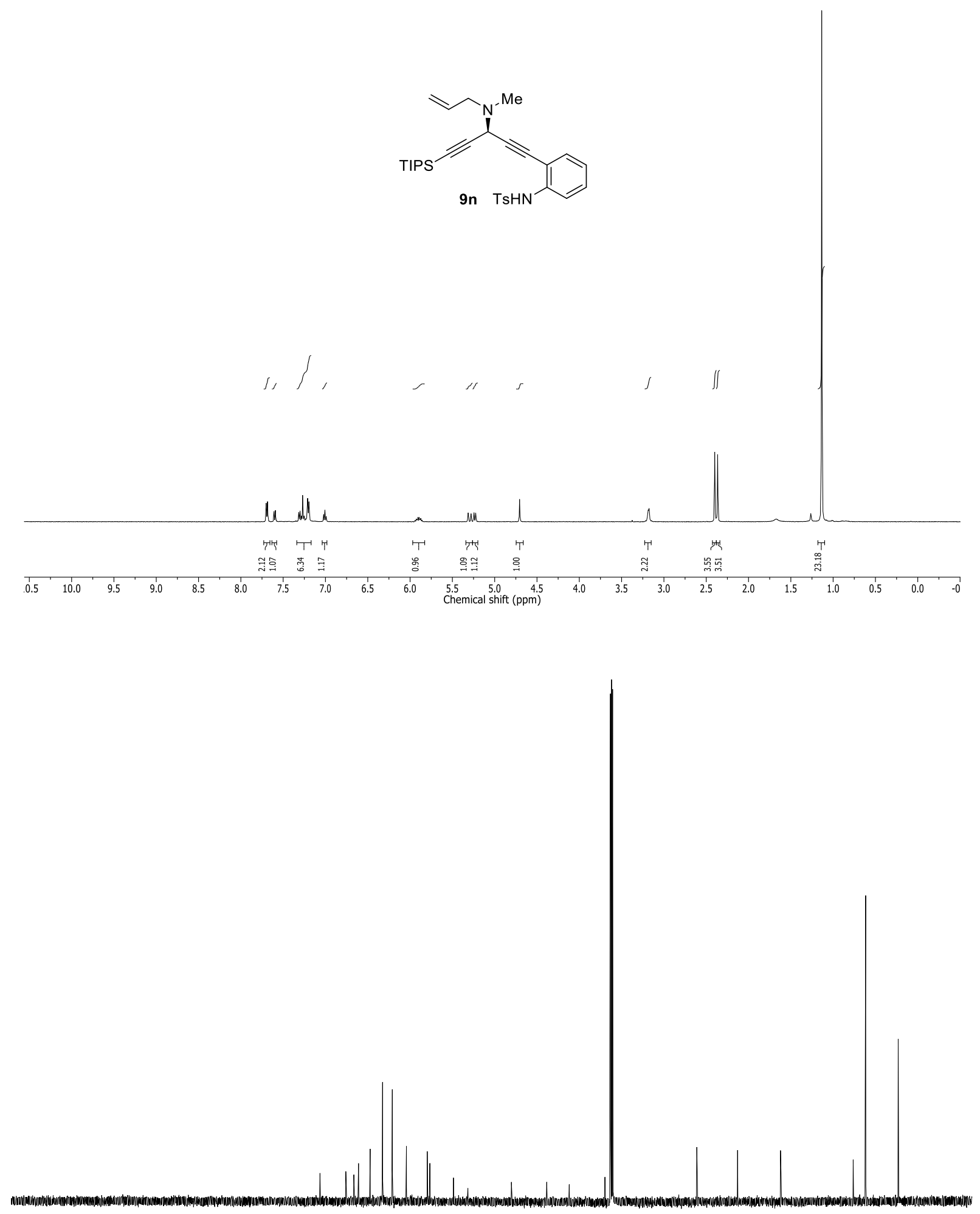

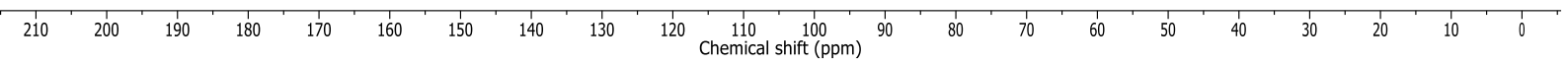



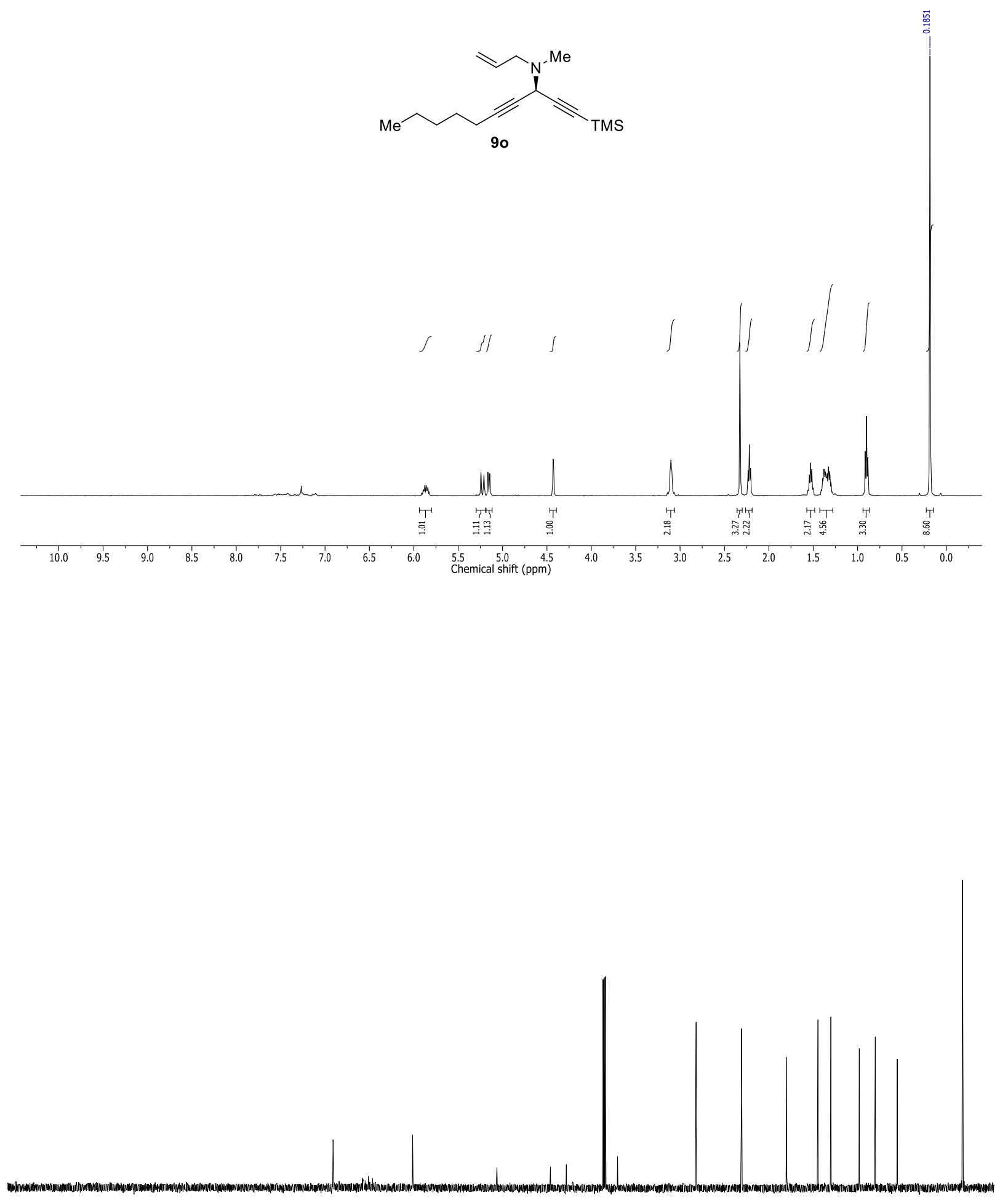

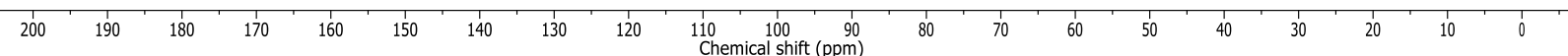



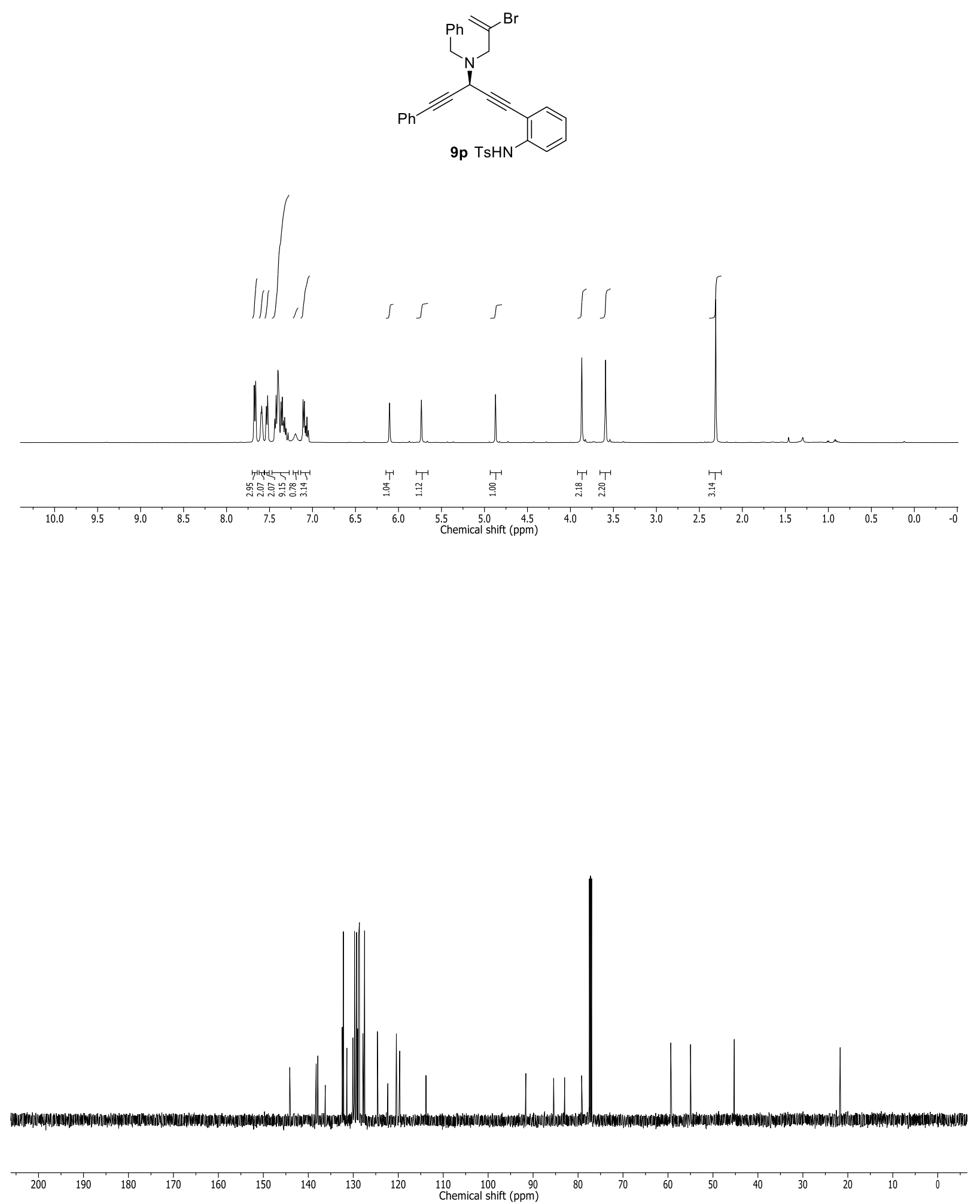

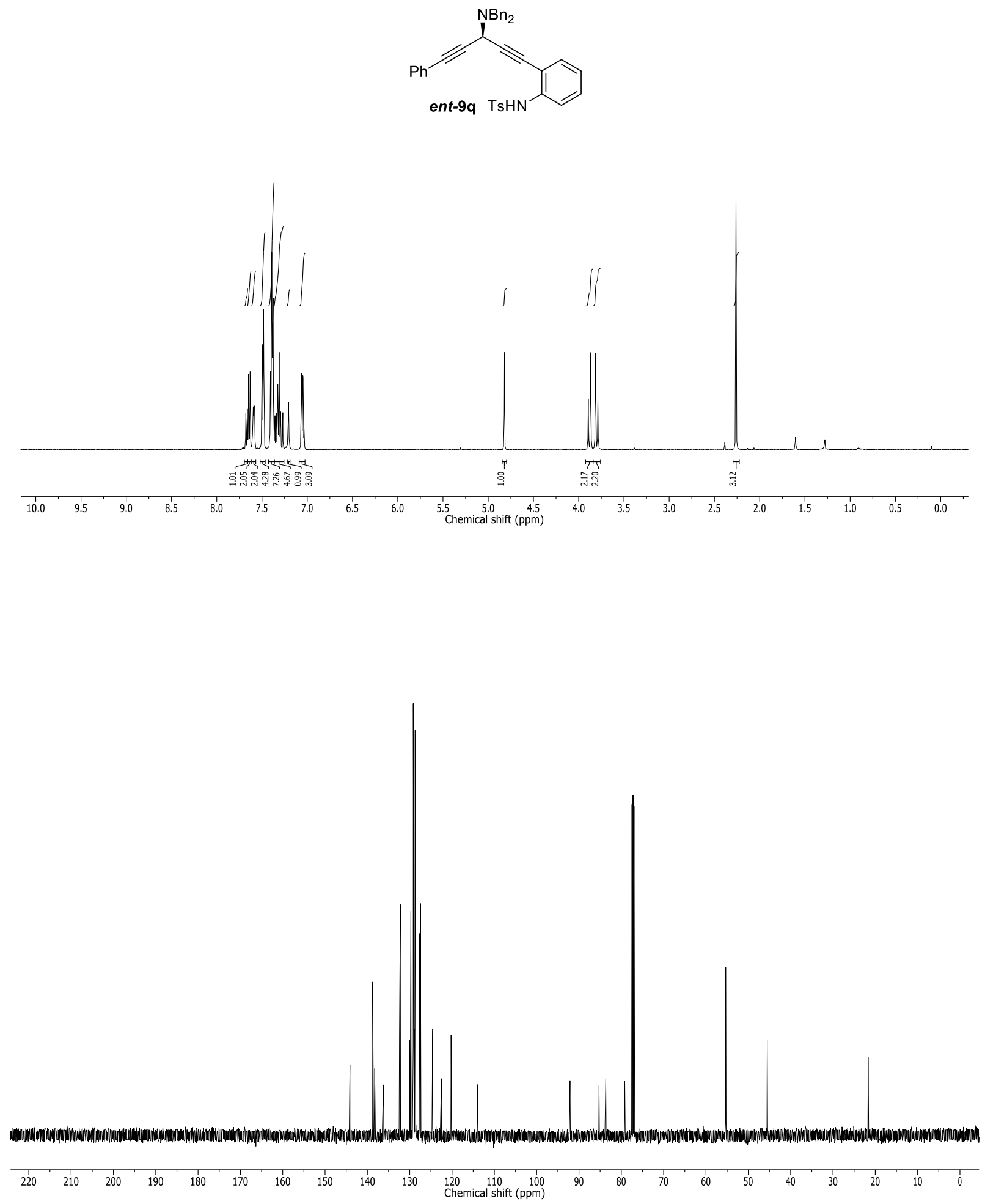
9. NMR Spectra of Compounds 10, 11, 13, 14, 16, 17, 18 and 4 

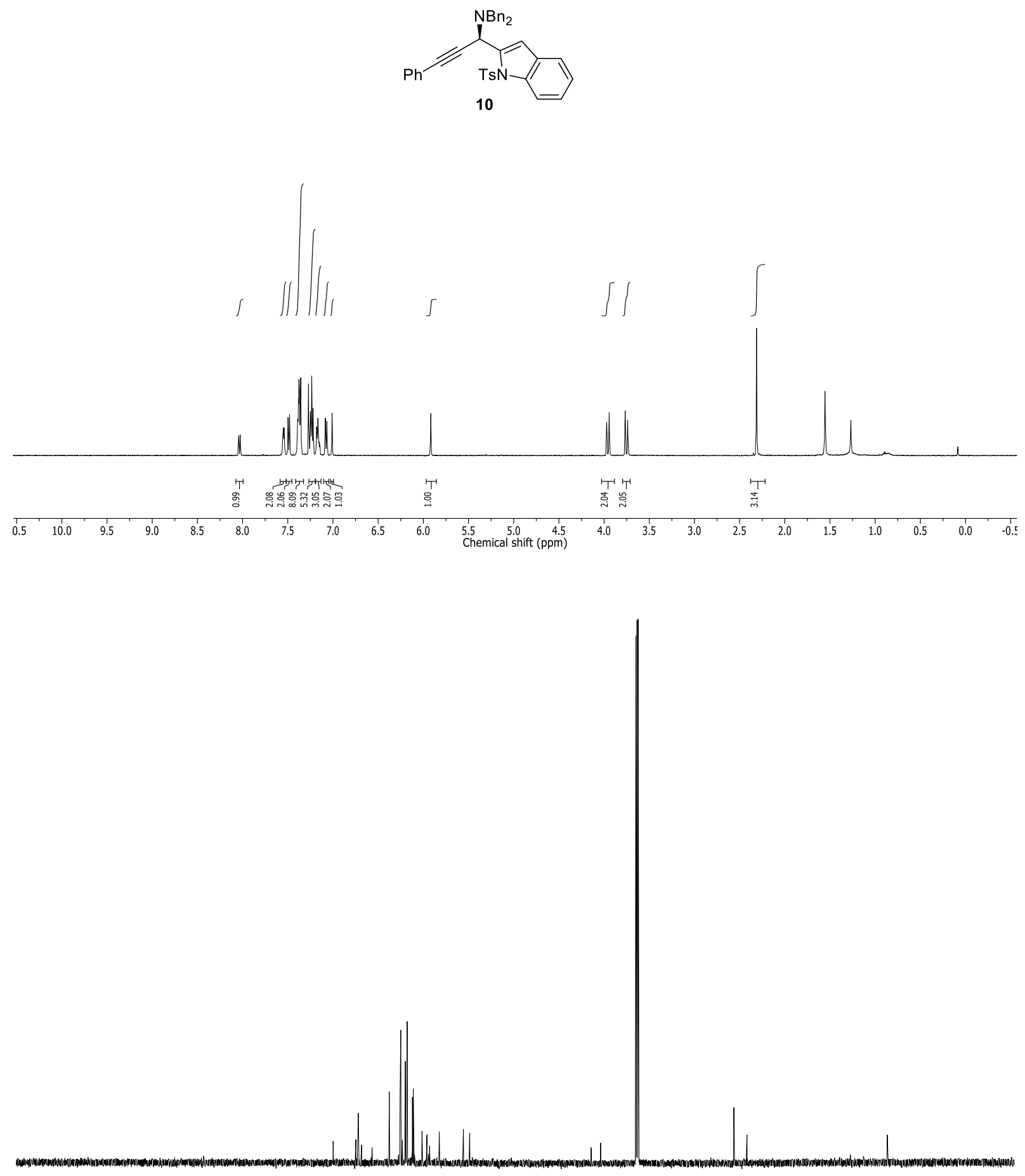

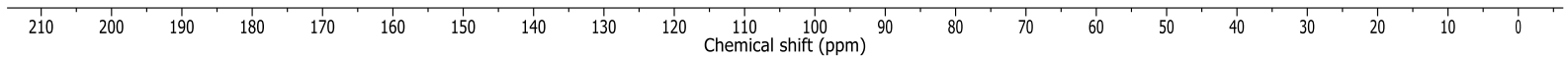



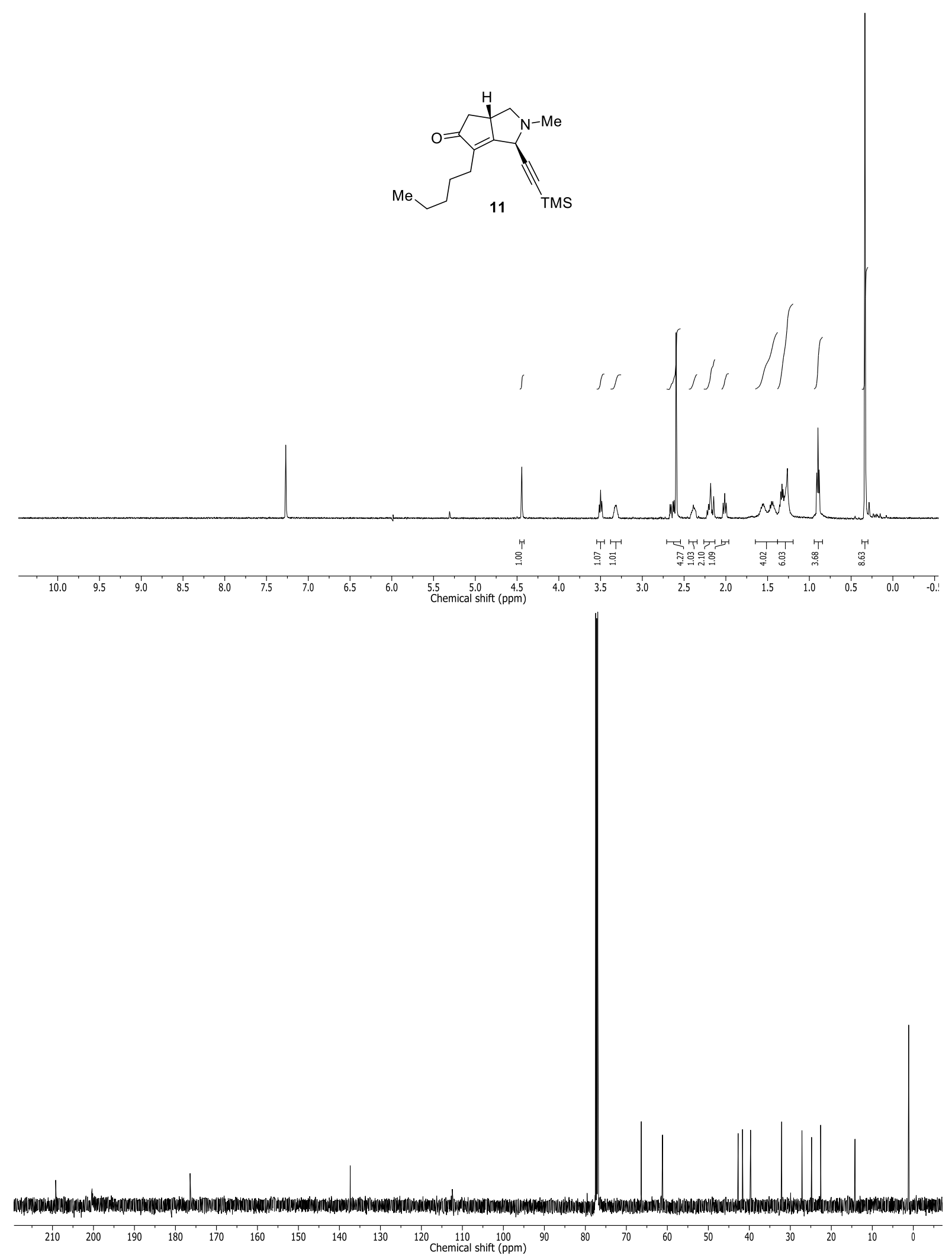

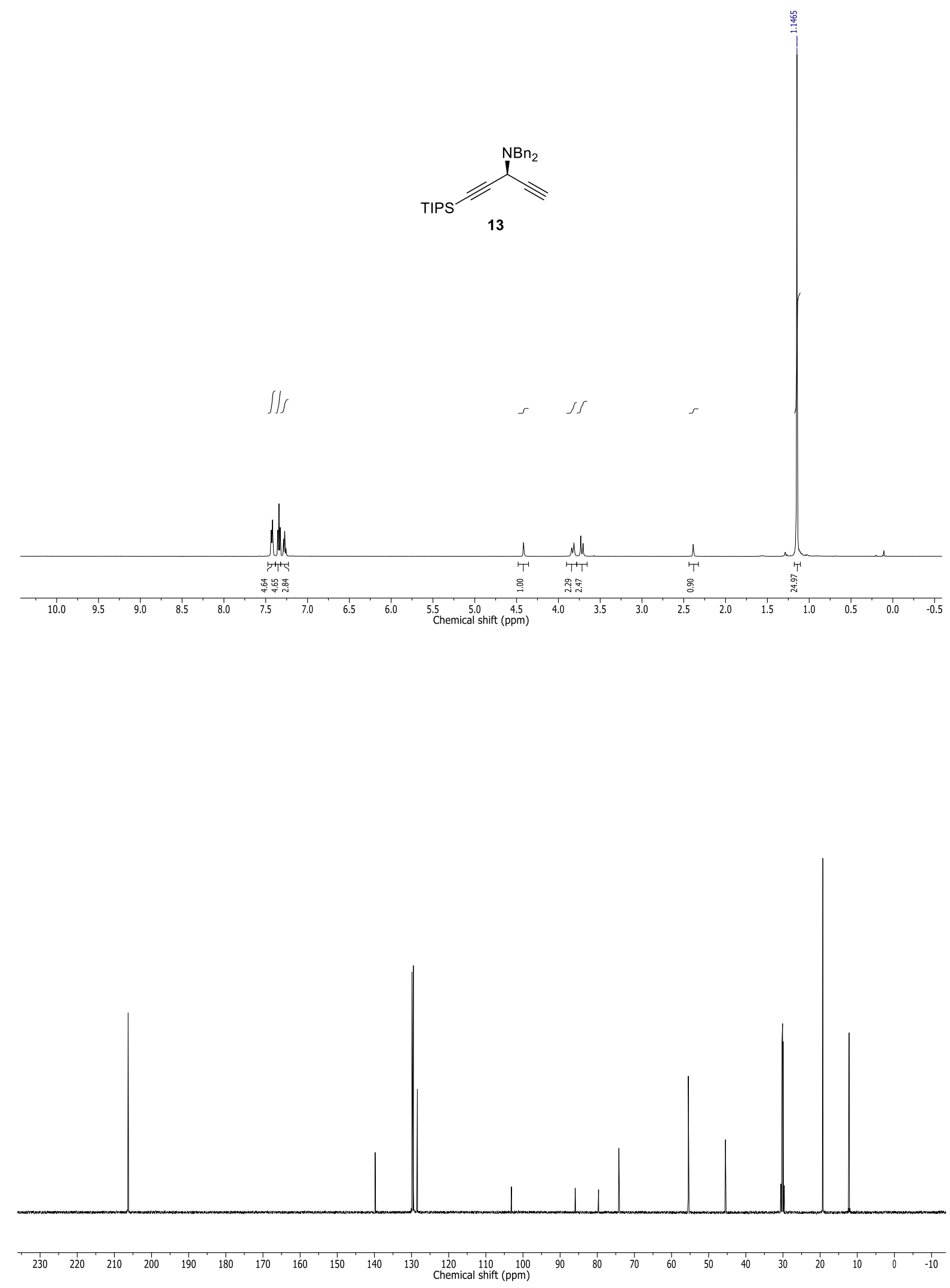

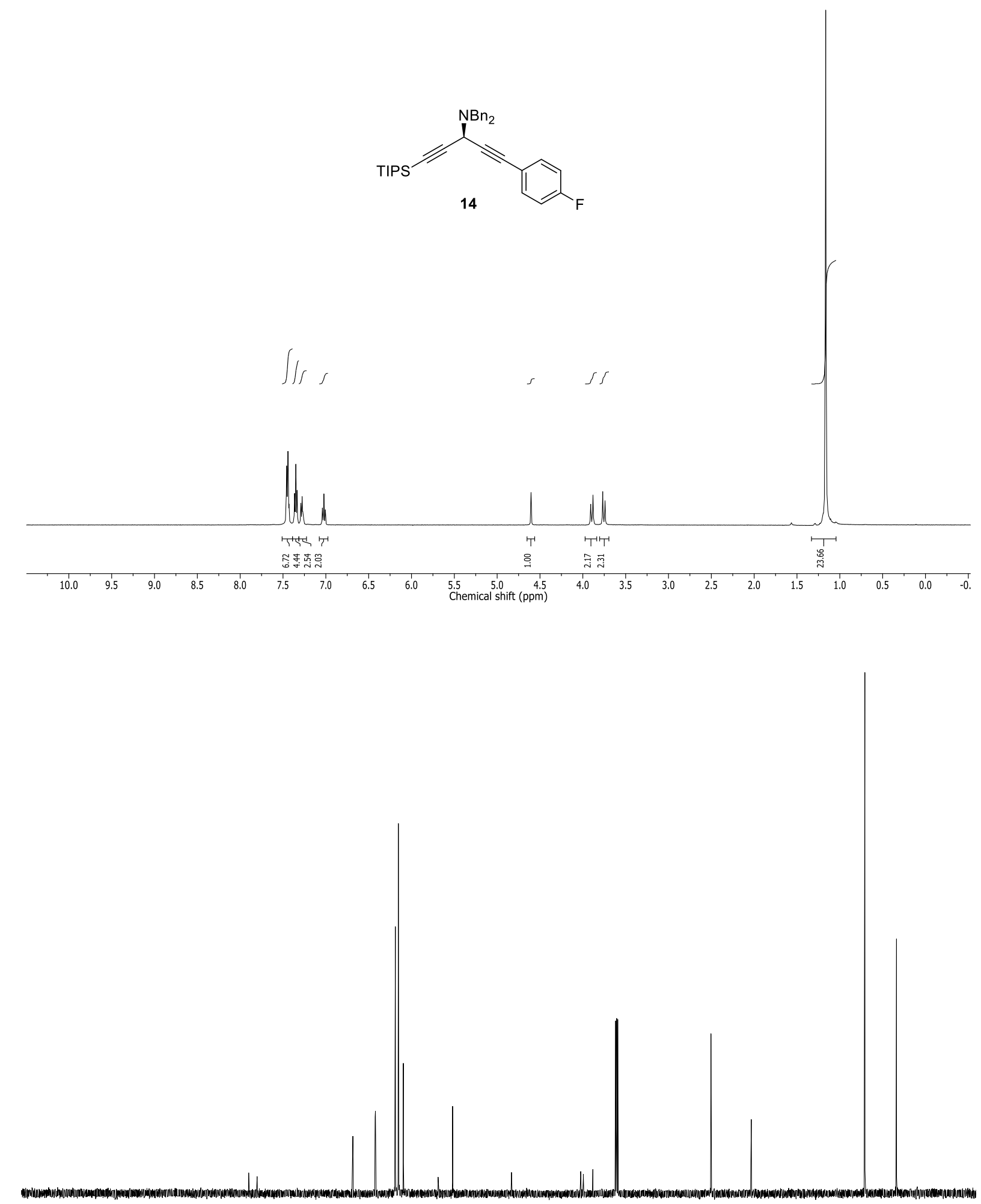

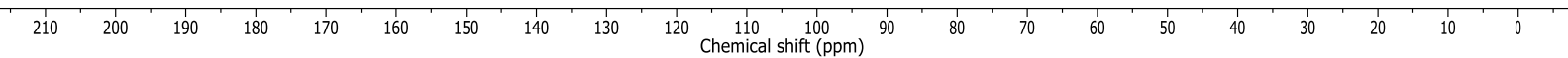



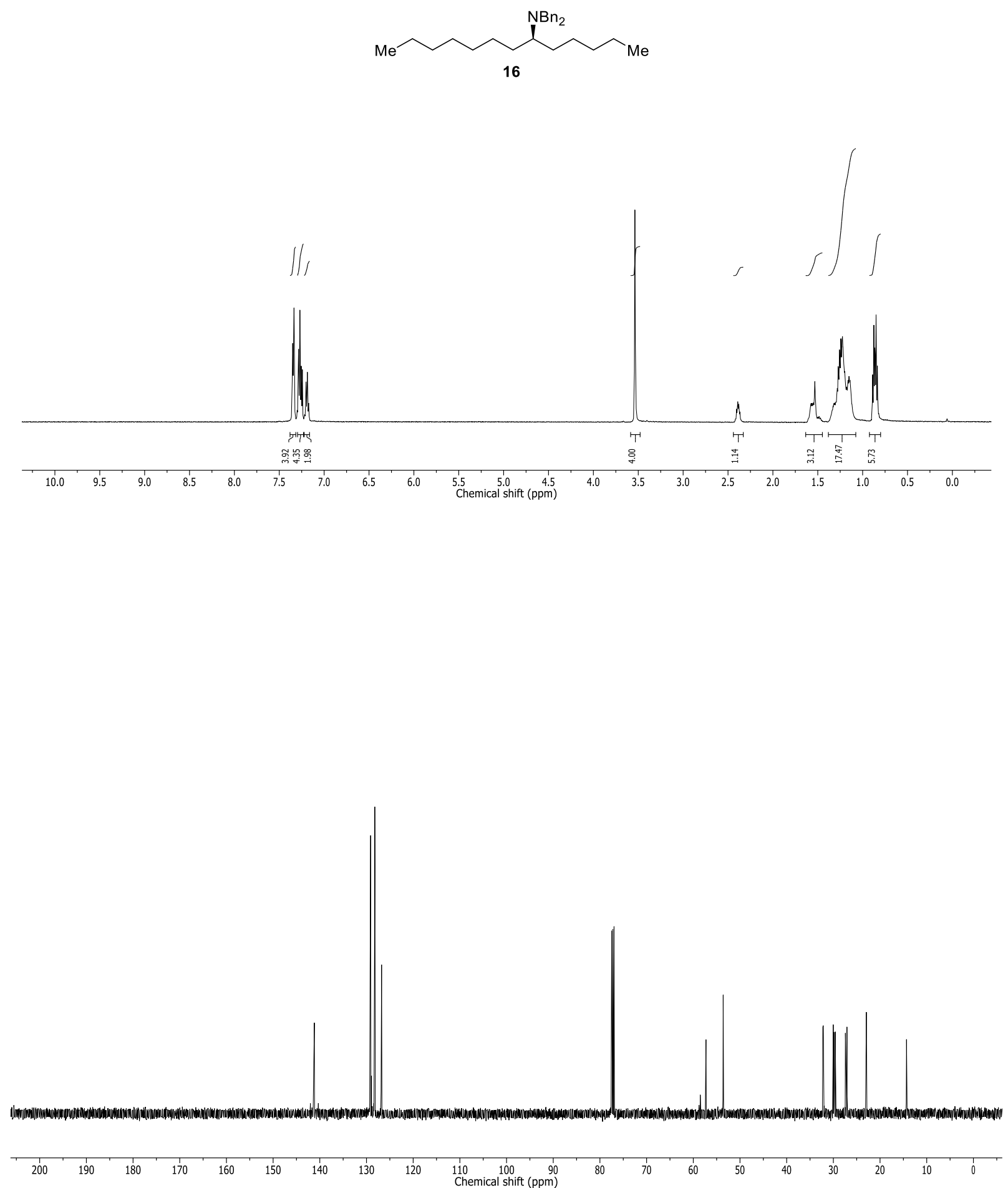

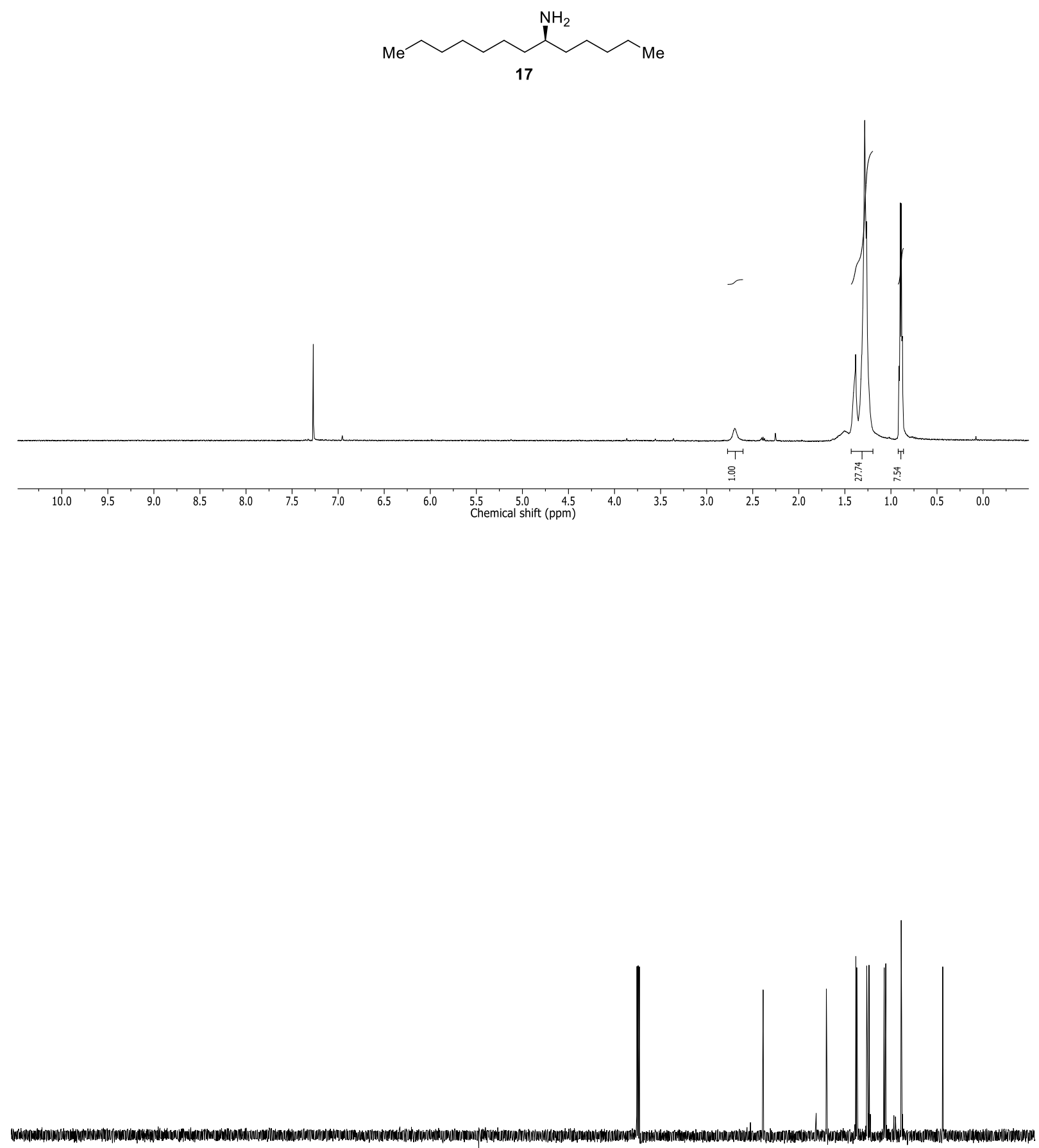

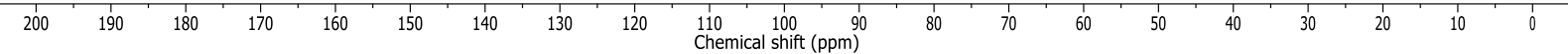



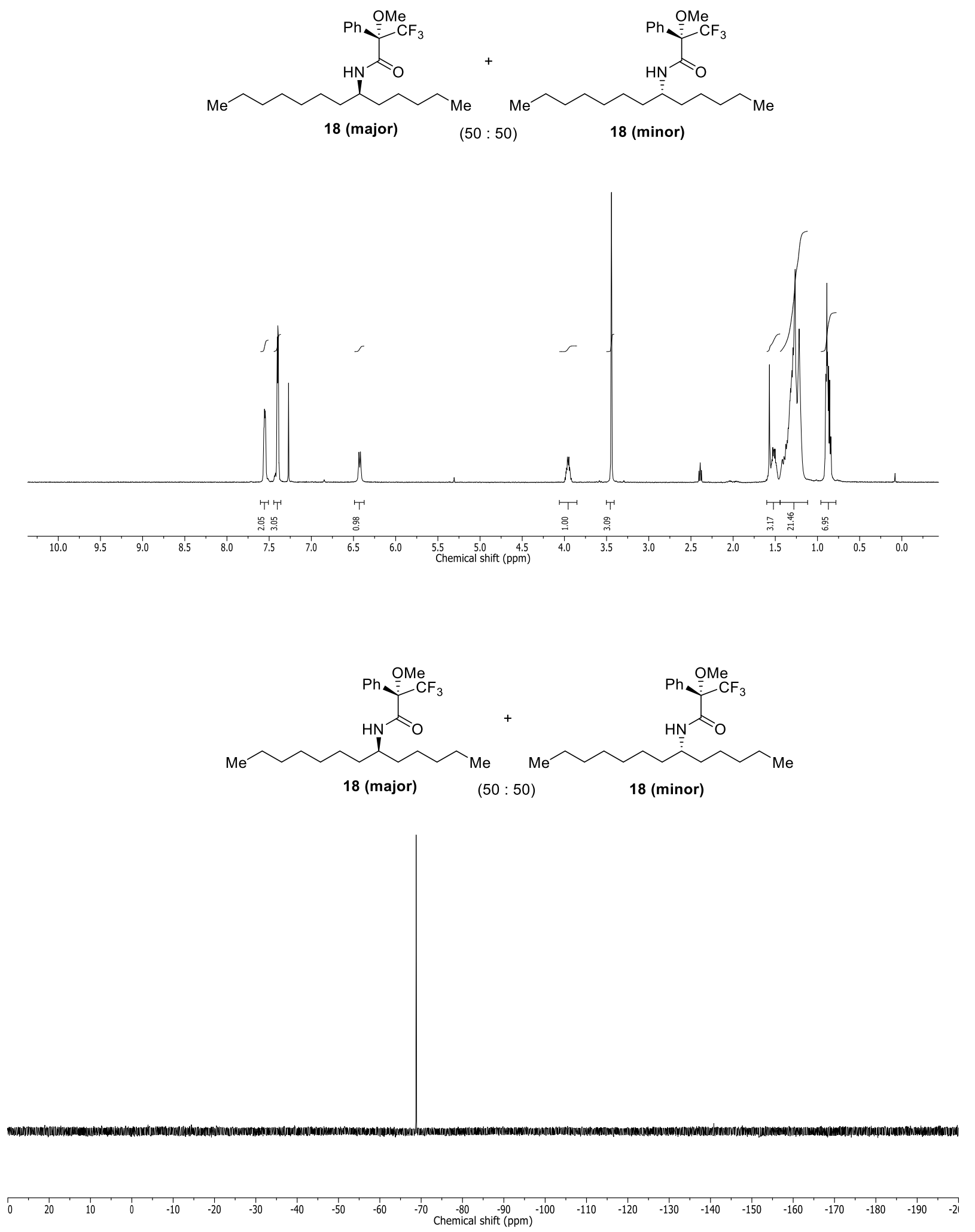

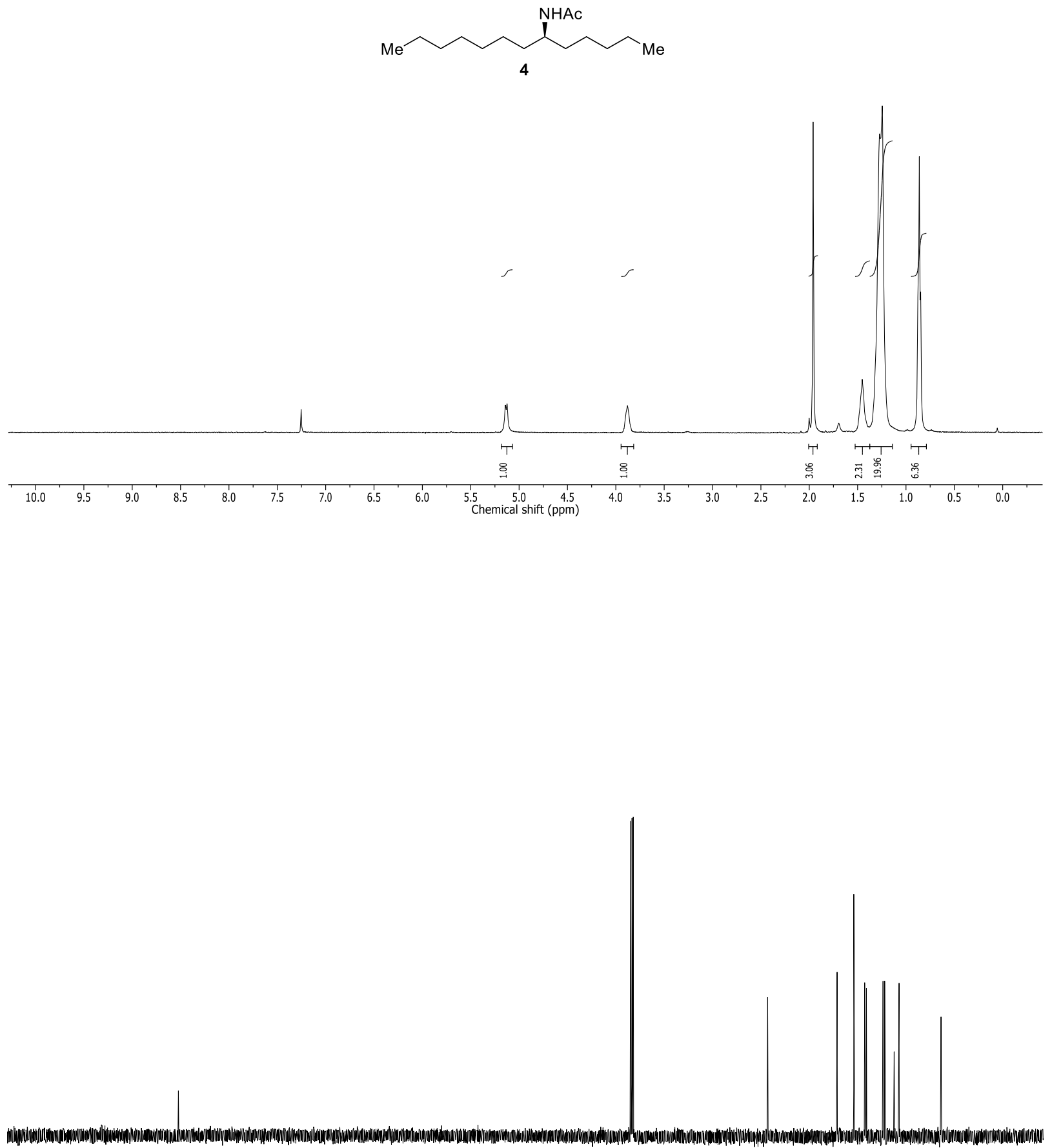

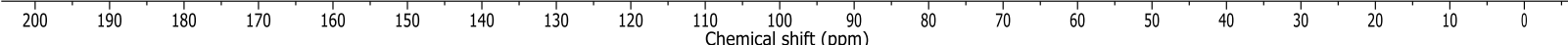




\section{X-Ray Crystallographic Data of Compound ent-9b}

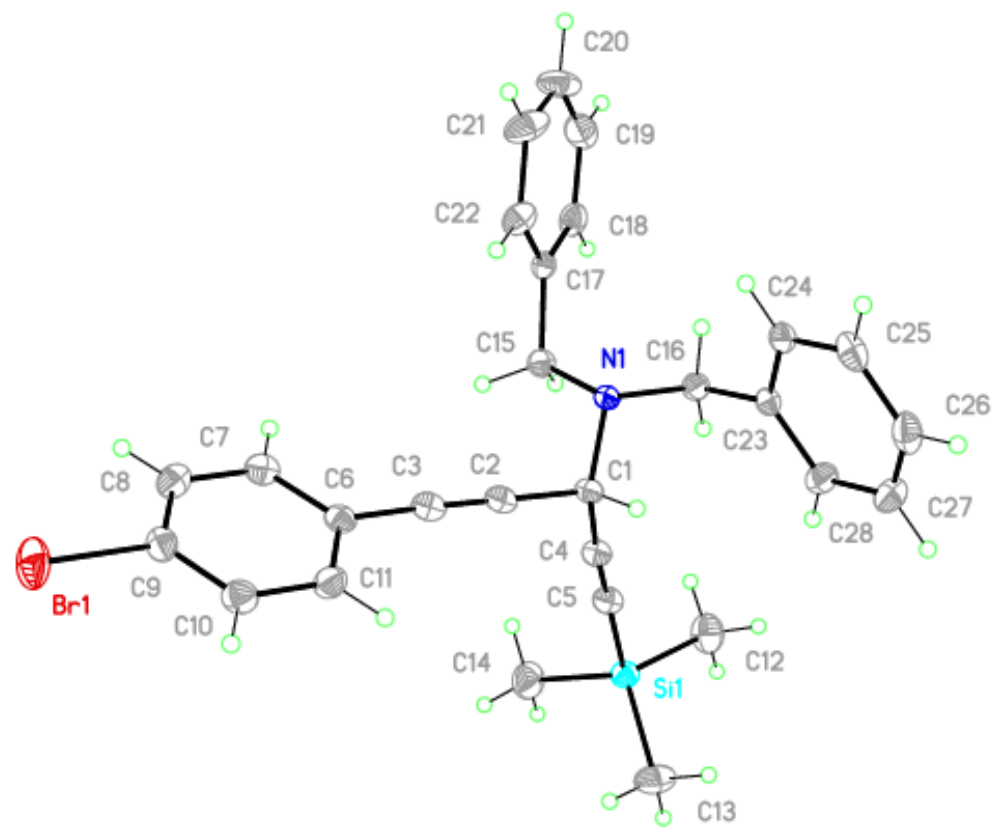

X-Ray experimental: The X-Ray quality single-crystals were grown from a $50: 50$ mixture of hexanes and dichloromethane. The amine ent-9b, $(25 \mathrm{mg}, 96 \%$ ee determined by Chiral HPLC, had a $(S)$-configuration and it was synthesized from $(\boldsymbol{R})$-StackPhos according to the general procedure described in section 3.1. For the recrystallization, the amine was placed in a vial and dissolved in dichloromethane (3 drops). Then hexanes $(1.0 \mathrm{~mL})$ were added to the vial. The vial was capped, and placed in the freezer at $-20^{\circ} \mathrm{C}$. Single-crystals were observed after one week.

X-Ray Intensity data were collected at $100 \mathrm{~K}$ on a Bruker DUO diffractometer using MoK $\alpha$ radiation $(\lambda=0.71073 \AA)$ and an APEXII CCD area detector.

Raw data frames were read by program SAINT and integrated using 3D profiling algorithms. The resulting data were reduced to produce hkl reflections and their intensities and 
estimated standard deviations. The data were corrected for Lorentz and polarization effects and numerical absorption corrections were applied based on indexed and measured faces.

The structure was solved and refined in SHELXTL2013, using full-matrix least-squares refinement. The non-H atoms were refined with anisotropic thermal parameters and all of the $\mathrm{H}$ atoms were calculated in idealized positions and refined riding on their parent atoms. The correct enantiomer is refined and reported here as can be evidenced by the value of the Flack $\mathrm{x}$ parameter of $-0.0131(12)$. In the final cycle of refinement, 5836 reflections (of which 5707 are observed with $\mathrm{I}>2 \sigma(\mathrm{I})$ ) were used to refine 283 parameters and the resulting $\mathrm{R}_{1}$, w $\mathrm{R}_{2}$ and $\mathrm{S}$ (goodness of fit) were $1.82 \%, 5.13 \%$ and 1.121 , respectively. The refinement was carried out by minimizing the $\mathrm{wR}_{2}$ function using $\mathrm{F}^{2}$ rather than $\mathrm{F}$ values. $\mathrm{R}_{1}$ is calculated to provide a reference to the conventional $\mathrm{R}$ value but its function is not minimized.

SHELXTL2013 (2013). Bruker-AXS, Madison, Wisconsin, USA.

\section{Datablock: paulo4}

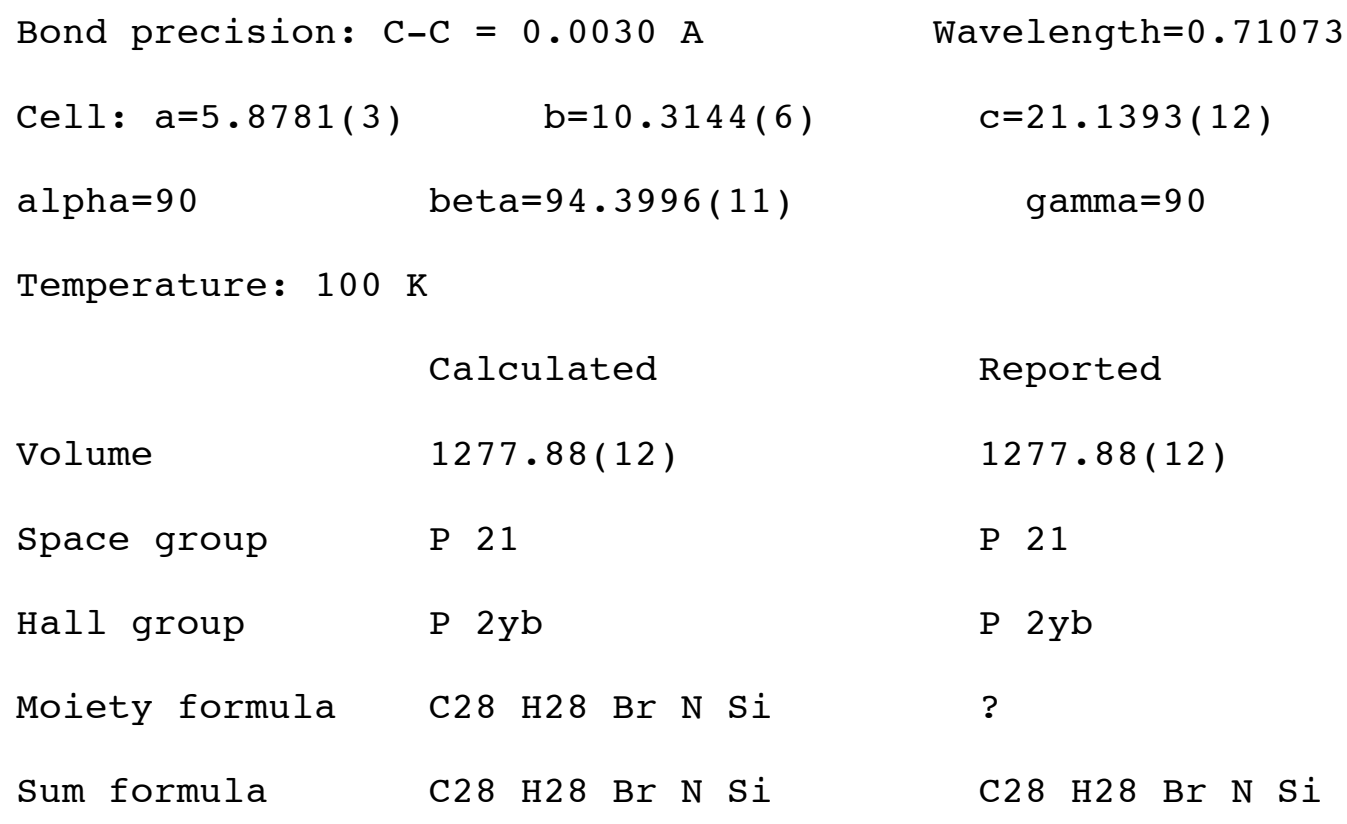




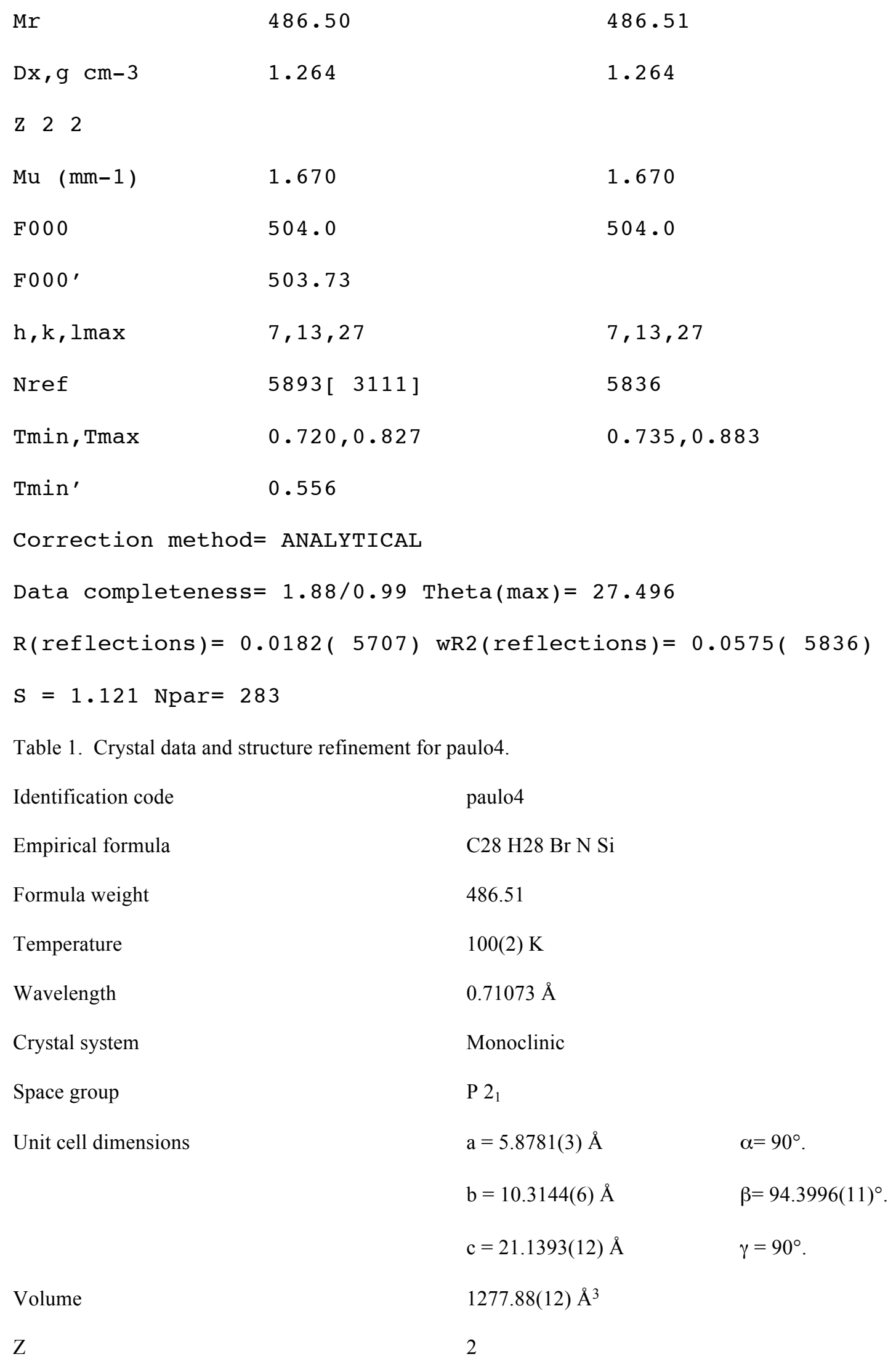


Density (calculated)

Absorption coefficient

$\mathrm{F}(000)$

Crystal size

Theta range for data collection

Index ranges

Reflections collected

Independent reflections

Completeness to theta $=25.242^{\circ}$

Absorption correction

Max. and min. transmission

Refinement method

Data / restraints / parameters

Goodness-of-fit on $\mathrm{F}^{2}$

Final $\mathrm{R}$ indices $[\mathrm{I}>2 \operatorname{sigma}(\mathrm{I})]$

$\mathrm{R}$ indices (all data)

Absolute structure parameter

Largest diff. peak and hole
$1.264 \mathrm{Mg} / \mathrm{m}^{3}$

$1.670 \mathrm{~mm}^{-1}$

504

$0.346 \times 0.169 \times 0.114 \mathrm{~mm}^{3}$

0.966 to $27.496^{\circ}$.

$-7 \leq \mathrm{h} \leq 7,-12 \leq \mathrm{k} \leq 13,-27 \leq \mathrm{l} \leq 27$

17946

$5836[\mathrm{R}(\mathrm{int})=0.0156]$

$99.9 \%$

Analytical

0.8830 and 0.7353

Full-matrix least-squares on $\mathrm{F}^{2}$

$5836 / 1 / 283$

1.121

$\mathrm{R} 1=0.0182, \mathrm{wR} 2=0.0513[5707]$

$\mathrm{R} 1=0.0193, \mathrm{wR} 2=0.0575$

$-0.0131(12)$

0.399 and -0.201 e. $\AA^{-3}$

$\mathrm{R} 1=\sum\left(|| \mathrm{F}_{\mathrm{O}}|-| \mathrm{F}_{\mathbf{c}} \mid\right) / \sum\left|\mathrm{F}_{\mathbf{O}}\right|$

$\mathrm{wR} 2=\left[\sum\left[\mathrm{w}\left(\mathrm{F}_{\mathrm{o}}{ }^{2}-\mathrm{F}_{\mathrm{c}}{ }^{2}\right)^{2}\right] / \sum\left[\mathrm{w}\left(\mathrm{F}_{\mathrm{o}}{ }^{2}\right)^{2}\right]\right]^{1 / 2}$

$\mathrm{S}=\left[\sum\left[\mathrm{w}\left(\mathrm{F}_{\mathrm{O}}^{2}-\mathrm{F}_{\mathrm{c}}{ }^{2}\right)^{2}\right] /(\mathrm{n}-\mathrm{p})\right]^{1 / 2}$

$\mathrm{w}=1 /\left[\sigma^{2}\left(\mathrm{~F}_{\mathrm{o}}{ }^{2}\right)+(\mathrm{m} * \mathrm{p})^{2}+\mathrm{n} * \mathrm{p}\right], \mathrm{p}=\left[\max \left(\mathrm{F}_{\mathrm{o}}^{2}, 0\right)+2^{*} \mathrm{~F}_{\mathrm{c}}{ }^{2}\right] / 3, \mathrm{~m} \& \mathrm{n}$ are constants. 
Table 2. Atomic coordinates $\left(\times 10^{4}\right)$ and equivalent isotropic displacement parameters $\left(\AA^{2} \times 10^{3}\right)$ for paulo4. $U(e q)$ is defined as one third of the trace of the orthogonalized $U^{i j}$ tensor.

\begin{tabular}{|c|c|c|c|c|}
\hline & $\mathrm{x}$ & $\mathrm{y}$ & z & $\mathrm{U}(\mathrm{eq})$ \\
\hline $\operatorname{Br}(1)$ & $1262(1)$ & $7069(1)$ & $388(1)$ & $36(1)$ \\
\hline $\operatorname{Si}(1)$ & $12306(1)$ & $14614(1)$ & $-1873(1)$ & $20(1)$ \\
\hline $\mathrm{N}(1)$ & $8564(2)$ & 10493(2) & $-3062(1)$ & $16(1)$ \\
\hline $\mathrm{C}(1)$ & $7606(3)$ & $11357(2)$ & $-2593(1)$ & $18(1)$ \\
\hline$C(2)$ & $6663(3)$ & $10601(2)$ & $-2084(1)$ & $19(1)$ \\
\hline$C(3)$ & $5849(3)$ & 9989(2) & $-1680(1)$ & $21(1)$ \\
\hline C(4) & $9204(3)$ & $12377(2)$ & $-2331(1)$ & $21(1)$ \\
\hline$C(5)$ & $10484(3)$ & $13242(2)$ & $-2156(1)$ & $23(1)$ \\
\hline$C(6)$ & $4798(3)$ & 9293(2) & $-1190(1)$ & $18(1)$ \\
\hline$C(7)$ & 5796(3) & $8169(2)$ & $-918(1)$ & $22(1)$ \\
\hline $\mathrm{C}(8)$ & $4772(3)$ & $7507(2)$ & $-446(1)$ & $24(1)$ \\
\hline$C(9)$ & $2726(3)$ & $7966(2)$ & $-250(1)$ & $22(1)$ \\
\hline$C(10)$ & 1691(3) & $9070(2)$ & $-512(1)$ & $24(1)$ \\
\hline$C(11)$ & 2733(3) & $9726(2)$ & $-983(1)$ & $23(1)$ \\
\hline$C(12)$ & $14064(4)$ & $15130(3)$ & $-2524(1)$ & $34(1)$ \\
\hline$C(13)$ & 10381(4) & $15948(2)$ & $-1655(1)$ & $32(1)$ \\
\hline$C(14)$ & $14153(4)$ & $14048(3)$ & $-1174(1)$ & $35(1)$ \\
\hline$C(15)$ & 10453(3) & $9681(2)$ & $-2786(1)$ & $18(1)$ \\
\hline$C(16)$ & $9258(3)$ & $11215(2)$ & $-3616(1)$ & $18(1)$ \\
\hline$C(17)$ & 10706(3) & $8529(2)$ & $-3211(1)$ & $17(1)$ \\
\hline$C(18)$ & $12614(3)$ & $8362(2)$ & $-3558(1)$ & $24(1)$ \\
\hline$C(19)$ & 12702(4) & $7341(2)$ & $-3986(1)$ & $33(1)$ \\
\hline$C(20)$ & $10925(4)$ & $6476(2)$ & $-4063(1)$ & $38(1)$ \\
\hline$C(21)$ & $9036(4)$ & $6614(2)$ & $-3712(1)$ & $35(1)$ \\
\hline$C(22)$ & $8932(3)$ & $7632(2)$ & $-3292(1)$ & $23(1)$ \\
\hline$C(23)$ & $7213(3)$ & $11785(2)$ & $-3991(1)$ & $18(1)$ \\
\hline$C(24)$ & $5670(3)$ & $10970(2)$ & $-4333(1)$ & $20(1)$ \\
\hline$C(25)$ & $3728(4)$ & $11472(2)$ & $-4660(1)$ & $25(1)$ \\
\hline$C(26)$ & $3300(4)$ & $12797(2)$ & $-4651(1)$ & $28(1)$ \\
\hline$C(27)$ & $4826(4)$ & $13610(2)$ & $-4319(1)$ & $31(1)$ \\
\hline$C(28)$ & $6780(4)$ & $13109(2)$ & $-3990(1)$ & $25(1)$ \\
\hline
\end{tabular}


Table 3. Bond lengths $[\AA]$ and angles $\left[{ }^{\circ}\right]$ for paulo4.

\begin{tabular}{|c|c|}
\hline $\operatorname{Br}(1)-C(9)$ & $1.8959(19)$ \\
\hline $\mathrm{Si}(1)-\mathrm{C}(5)$ & $1.846(2)$ \\
\hline $\operatorname{Si}(1)-C(14)$ & $1.859(2)$ \\
\hline $\operatorname{Si}(1)-C(12)$ & $1.860(2)$ \\
\hline $\operatorname{Si}(1)-C(13)$ & $1.861(2)$ \\
\hline N(1)-C(16) & $1.471(2)$ \\
\hline $\mathrm{N}(1)-\mathrm{C}(15)$ & $1.475(2)$ \\
\hline $\mathrm{N}(1)-\mathrm{C}(1)$ & $1.477(2)$ \\
\hline $\mathrm{C}(1)-\mathrm{C}(2)$ & $1.471(3)$ \\
\hline$C(1)-C(4)$ & $1.490(3)$ \\
\hline $\mathrm{C}(1)-\mathrm{H}(1 \mathrm{~A})$ & 1.0000 \\
\hline$C(2)-C(3)$ & $1.192(3)$ \\
\hline$C(3)-C(6)$ & $1.438(3)$ \\
\hline$C(4)-C(5)$ & $1.206(3)$ \\
\hline $\mathrm{C}(6)-\mathrm{C}(11)$ & $1.395(3)$ \\
\hline$C(6)-C(7)$ & $1.403(3)$ \\
\hline$C(7)-C(8)$ & $1.384(3)$ \\
\hline $\mathrm{C}(7)-\mathrm{H}(7 \mathrm{~A})$ & 0.9500 \\
\hline $\mathrm{C}(8)-\mathrm{C}(9)$ & $1.385(3)$ \\
\hline $\mathrm{C}(8)-\mathrm{H}(8 \mathrm{~A})$ & 0.9500 \\
\hline $\mathrm{C}(9)-\mathrm{C}(10)$ & $1.386(3)$ \\
\hline$C(10)-C(11)$ & $1.386(3)$ \\
\hline $\mathrm{C}(10)-\mathrm{H}(10 \mathrm{~A})$ & 0.9500 \\
\hline $\mathrm{C}(11)-\mathrm{H}(11 \mathrm{~A})$ & 0.9500 \\
\hline $\mathrm{C}(12)-\mathrm{H}(12 \mathrm{~A})$ & 0.9800 \\
\hline $\mathrm{C}(12)-\mathrm{H}(12 \mathrm{~B})$ & 0.9800 \\
\hline $\mathrm{C}(12)-\mathrm{H}(12 \mathrm{C})$ & 0.9800 \\
\hline $\mathrm{C}(13)-\mathrm{H}(13 \mathrm{~A})$ & 0.9800 \\
\hline $\mathrm{C}(13)-\mathrm{H}(13 \mathrm{~B})$ & 0.9800 \\
\hline $\mathrm{C}(13)-\mathrm{H}(13 \mathrm{C})$ & 0.9800 \\
\hline $\mathrm{C}(14)-\mathrm{H}(14 \mathrm{~A})$ & 0.9800 \\
\hline $\mathrm{C}(14)-\mathrm{H}(14 \mathrm{~B})$ & 0.9800 \\
\hline $\mathrm{C}(14)-\mathrm{H}(14 \mathrm{C})$ & 0.9800 \\
\hline$C(15)-C(17)$ & $1.504(3)$ \\
\hline
\end{tabular}




\begin{tabular}{|c|c|}
\hline $\mathrm{C}(15)-\mathrm{H}(15 \mathrm{~A})$ & 0.9900 \\
\hline $\mathrm{C}(15)-\mathrm{H}(15 \mathrm{~B})$ & 0.9900 \\
\hline$C(16)-C(23)$ & $1.508(2)$ \\
\hline $\mathrm{C}(16)-\mathrm{H}(16 \mathrm{~A})$ & 0.9900 \\
\hline $\mathrm{C}(16)-\mathrm{H}(16 \mathrm{~B})$ & 0.9900 \\
\hline$C(17)-C(22)$ & $1.394(3)$ \\
\hline$C(17)-C(18)$ & $1.396(3)$ \\
\hline C(18)-C(19) & $1.393(3)$ \\
\hline $\mathrm{C}(18)-\mathrm{H}(18 \mathrm{~A})$ & 0.9500 \\
\hline$C(19)-C(20)$ & $1.374(4)$ \\
\hline C(19)-H(19A) & 0.9500 \\
\hline$C(20)-C(21)$ & $1.389(4)$ \\
\hline $\mathrm{C}(20)-\mathrm{H}(20 \mathrm{~A})$ & 0.9500 \\
\hline$C(21)-C(22)$ & $1.379(3)$ \\
\hline $\mathrm{C}(21)-\mathrm{H}(21 \mathrm{~A})$ & 0.9500 \\
\hline $\mathrm{C}(22)-\mathrm{H}(22 \mathrm{~A})$ & 0.9500 \\
\hline$C(23)-C(28)$ & $1.390(3)$ \\
\hline$C(23)-C(24)$ & $1.396(3)$ \\
\hline$C(24)-C(25)$ & $1.389(3)$ \\
\hline $\mathrm{C}(24)-\mathrm{H}(24 \mathrm{~A})$ & 0.9500 \\
\hline$C(25)-C(26)$ & $1.390(3)$ \\
\hline $\mathrm{C}(25)-\mathrm{H}(25 \mathrm{~A})$ & 0.9500 \\
\hline$C(26)-C(27)$ & $1.379(3)$ \\
\hline $\mathrm{C}(26)-\mathrm{H}(26 \mathrm{~A})$ & 0.9500 \\
\hline$C(27)-C(28)$ & $1.396(3)$ \\
\hline $\mathrm{C}(27)-\mathrm{H}(27 \mathrm{~A})$ & 0.9500 \\
\hline $\mathrm{C}(28)-\mathrm{H}(28 \mathrm{~A})$ & 0.9500 \\
\hline$C(5)-\operatorname{Si}(1)-C(14)$ & $107.75(11)$ \\
\hline $\mathrm{C}(5)-\mathrm{Si}(1)-\mathrm{C}(12)$ & $108.79(10)$ \\
\hline $\mathrm{C}(14)-\mathrm{Si}(1)-\mathrm{C}(12)$ & $110.68(11)$ \\
\hline$C(5)-\operatorname{Si}(1)-C(13)$ & $107.34(9)$ \\
\hline $\mathrm{C}(14)-\mathrm{Si}(1)-\mathrm{C}(13)$ & $111.34(12)$ \\
\hline $\mathrm{C}(12)-\mathrm{Si}(1)-\mathrm{C}(13)$ & $110.79(12)$ \\
\hline $\mathrm{C}(16)-\mathrm{N}(1)-\mathrm{C}(15)$ & $110.84(14)$ \\
\hline $\mathrm{C}(16)-\mathrm{N}(1)-\mathrm{C}(1)$ & $111.81(14)$ \\
\hline
\end{tabular}




\begin{tabular}{|c|c|}
\hline $\mathrm{C}(15)-\mathrm{N}(1)-\mathrm{C}(1)$ & $113.03(14)$ \\
\hline $\mathrm{C}(2)-\mathrm{C}(1)-\mathrm{N}(1)$ & $110.83(15)$ \\
\hline$C(2)-C(1)-C(4)$ & $111.36(15)$ \\
\hline $\mathrm{N}(1)-\mathrm{C}(1)-\mathrm{C}(4)$ & $114.49(15)$ \\
\hline $\mathrm{C}(2)-\mathrm{C}(1)-\mathrm{H}(1 \mathrm{~A})$ & 106.5 \\
\hline $\mathrm{N}(1)-\mathrm{C}(1)-\mathrm{H}(1 \mathrm{~A})$ & 106.5 \\
\hline $\mathrm{C}(4)-\mathrm{C}(1)-\mathrm{H}(1 \mathrm{~A})$ & 106.5 \\
\hline$C(3)-C(2)-C(1)$ & $178.4(2)$ \\
\hline$C(2)-C(3)-C(6)$ & $177.7(2)$ \\
\hline$C(5)-C(4)-C(1)$ & $175.8(2)$ \\
\hline$C(4)-C(5)-S i(1)$ & $176.86(18)$ \\
\hline$C(11)-C(6)-C(7)$ & $118.84(18)$ \\
\hline$C(11)-C(6)-C(3)$ & $119.85(18)$ \\
\hline$C(7)-C(6)-C(3)$ & $121.30(17)$ \\
\hline$C(8)-C(7)-C(6)$ & $120.82(18)$ \\
\hline $\mathrm{C}(8)-\mathrm{C}(7)-\mathrm{H}(7 \mathrm{~A})$ & 119.6 \\
\hline $\mathrm{C}(6)-\mathrm{C}(7)-\mathrm{H}(7 \mathrm{~A})$ & 119.6 \\
\hline $\mathrm{C}(7)-\mathrm{C}(8)-\mathrm{C}(9)$ & 118.82(19) \\
\hline $\mathrm{C}(7)-\mathrm{C}(8)-\mathrm{H}(8 \mathrm{~A})$ & 120.6 \\
\hline $\mathrm{C}(9)-\mathrm{C}(8)-\mathrm{H}(8 \mathrm{~A})$ & 120.6 \\
\hline$C(8)-C(9)-C(10)$ & $121.78(19)$ \\
\hline$C(8)-C(9)-B r(1)$ & $119.85(16)$ \\
\hline$C(10)-C(9)-B r(1)$ & $118.37(15)$ \\
\hline $\mathrm{C}(9)-\mathrm{C}(10)-\mathrm{C}(11)$ & $118.92(18)$ \\
\hline $\mathrm{C}(9)-\mathrm{C}(10)-\mathrm{H}(10 \mathrm{~A})$ & 120.5 \\
\hline $\mathrm{C}(11)-\mathrm{C}(10)-\mathrm{H}(10 \mathrm{~A})$ & 120.5 \\
\hline$C(10)-C(11)-C(6)$ & $120.80(19)$ \\
\hline $\mathrm{C}(10)-\mathrm{C}(11)-\mathrm{H}(11 \mathrm{~A})$ & 119.6 \\
\hline $\mathrm{C}(6)-\mathrm{C}(11)-\mathrm{H}(11 \mathrm{~A})$ & 119.6 \\
\hline $\mathrm{Si}(1)-\mathrm{C}(12)-\mathrm{H}(12 \mathrm{~A})$ & 109.5 \\
\hline $\mathrm{Si}(1)-\mathrm{C}(12)-\mathrm{H}(12 \mathrm{~B})$ & 109.5 \\
\hline $\mathrm{H}(12 \mathrm{~A})-\mathrm{C}(12)-\mathrm{H}(12 \mathrm{~B})$ & 109.5 \\
\hline $\mathrm{Si}(1)-\mathrm{C}(12)-\mathrm{H}(12 \mathrm{C})$ & 109.5 \\
\hline $\mathrm{H}(12 \mathrm{~A})-\mathrm{C}(12)-\mathrm{H}(12 \mathrm{C})$ & 109.5 \\
\hline $\mathrm{H}(12 \mathrm{~B})-\mathrm{C}(12)-\mathrm{H}(12 \mathrm{C})$ & 109.5 \\
\hline $\mathrm{Si}(1)-\mathrm{C}(13)-\mathrm{H}(13 \mathrm{~A})$ & 109.5 \\
\hline
\end{tabular}




\begin{tabular}{|c|c|}
\hline $\mathrm{Si}(1)-\mathrm{C}(13)-\mathrm{H}(13 \mathrm{~B})$ & 109.5 \\
\hline $\mathrm{H}(13 \mathrm{~A})-\mathrm{C}(13)-\mathrm{H}(13 \mathrm{~B})$ & 109.5 \\
\hline $\mathrm{Si}(1)-\mathrm{C}(13)-\mathrm{H}(13 \mathrm{C})$ & 109.5 \\
\hline $\mathrm{H}(13 \mathrm{~A})-\mathrm{C}(13)-\mathrm{H}(13 \mathrm{C})$ & 109.5 \\
\hline $\mathrm{H}(13 \mathrm{~B})-\mathrm{C}(13)-\mathrm{H}(13 \mathrm{C})$ & 109.5 \\
\hline $\mathrm{Si}(1)-\mathrm{C}(14)-\mathrm{H}(14 \mathrm{~A})$ & 109.5 \\
\hline Si(1)-C(14)-H(14B) & 109.5 \\
\hline $\mathrm{H}(14 \mathrm{~A})-\mathrm{C}(14)-\mathrm{H}(14 \mathrm{~B})$ & 109.5 \\
\hline $\mathrm{Si}(1)-\mathrm{C}(14)-\mathrm{H}(14 \mathrm{C})$ & 109.5 \\
\hline $\mathrm{H}(14 \mathrm{~A})-\mathrm{C}(14)-\mathrm{H}(14 \mathrm{C})$ & 109.5 \\
\hline $\mathrm{H}(14 \mathrm{~B})-\mathrm{C}(14)-\mathrm{H}(14 \mathrm{C})$ & 109.5 \\
\hline $\mathrm{N}(1)-\mathrm{C}(15)-\mathrm{C}(17)$ & $108.55(14)$ \\
\hline $\mathrm{N}(1)-\mathrm{C}(15)-\mathrm{H}(15 \mathrm{~A})$ & 110.0 \\
\hline $\mathrm{C}(17)-\mathrm{C}(15)-\mathrm{H}(15 \mathrm{~A})$ & 110.0 \\
\hline $\mathrm{N}(1)-\mathrm{C}(15)-\mathrm{H}(15 \mathrm{~B})$ & 110.0 \\
\hline $\mathrm{C}(17)-\mathrm{C}(15)-\mathrm{H}(15 \mathrm{~B})$ & 110.0 \\
\hline $\mathrm{H}(15 \mathrm{~A})-\mathrm{C}(15)-\mathrm{H}(15 \mathrm{~B})$ & 108.4 \\
\hline $\mathrm{N}(1)-\mathrm{C}(16)-\mathrm{C}(23)$ & $110.96(14)$ \\
\hline $\mathrm{N}(1)-\mathrm{C}(16)-\mathrm{H}(16 \mathrm{~A})$ & 109.4 \\
\hline $\mathrm{C}(23)-\mathrm{C}(16)-\mathrm{H}(16 \mathrm{~A})$ & 109.4 \\
\hline $\mathrm{N}(1)-\mathrm{C}(16)-\mathrm{H}(16 \mathrm{~B})$ & 109.4 \\
\hline $\mathrm{C}(23)-\mathrm{C}(16)-\mathrm{H}(16 \mathrm{~B})$ & 109.4 \\
\hline $\mathrm{H}(16 \mathrm{~A})-\mathrm{C}(16)-\mathrm{H}(16 \mathrm{~B})$ & 108.0 \\
\hline $\mathrm{C}(22)-\mathrm{C}(17)-\mathrm{C}(18)$ & $118.49(18)$ \\
\hline $\mathrm{C}(22)-\mathrm{C}(17)-\mathrm{C}(15)$ & $119.37(17)$ \\
\hline$C(18)-C(17)-C(15)$ & $122.04(18)$ \\
\hline C(19)-C(18)-C(17) & $120.4(2)$ \\
\hline $\mathrm{C}(19)-\mathrm{C}(18)-\mathrm{H}(18 \mathrm{~A})$ & 119.8 \\
\hline $\mathrm{C}(17)-\mathrm{C}(18)-\mathrm{H}(18 \mathrm{~A})$ & 119.8 \\
\hline $\mathrm{C}(20)-\mathrm{C}(19)-\mathrm{C}(18)$ & $120.1(2)$ \\
\hline $\mathrm{C}(20)-\mathrm{C}(19)-\mathrm{H}(19 \mathrm{~A})$ & 119.9 \\
\hline $\mathrm{C}(18)-\mathrm{C}(19)-\mathrm{H}(19 \mathrm{~A})$ & 119.9 \\
\hline$C(19)-C(20)-C(21)$ & $120.2(2)$ \\
\hline $\mathrm{C}(19)-\mathrm{C}(20)-\mathrm{H}(20 \mathrm{~A})$ & 119.9 \\
\hline $\mathrm{C}(21)-\mathrm{C}(20)-\mathrm{H}(20 \mathrm{~A})$ & 119.9 \\
\hline$C(22)-C(21)-C(20)$ & $119.9(2)$ \\
\hline
\end{tabular}




\begin{tabular}{ll}
$\mathrm{C}(22)-\mathrm{C}(21)-\mathrm{H}(21 \mathrm{~A})$ & 120.1 \\
$\mathrm{C}(20)-\mathrm{C}(21)-\mathrm{H}(21 \mathrm{~A})$ & 120.1 \\
$\mathrm{C}(21)-\mathrm{C}(22)-\mathrm{C}(17)$ & $120.97(19)$ \\
$\mathrm{C}(21)-\mathrm{C}(22)-\mathrm{H}(22 \mathrm{~A})$ & 119.5 \\
$\mathrm{C}(17)-\mathrm{C}(22)-\mathrm{H}(22 \mathrm{~A})$ & 119.5 \\
$\mathrm{C}(28)-\mathrm{C}(23)-\mathrm{C}(24)$ & $118.77(18)$ \\
$\mathrm{C}(28)-\mathrm{C}(23)-\mathrm{C}(16)$ & $121.35(18)$ \\
$\mathrm{C}(24)-\mathrm{C}(23)-\mathrm{C}(16)$ & $119.85(17)$ \\
$\mathrm{C}(25)-\mathrm{C}(24)-\mathrm{C}(23)$ & $120.65(19)$ \\
$\mathrm{C}(25)-\mathrm{C}(24)-\mathrm{H}(24 \mathrm{~A})$ & 119.7 \\
$\mathrm{C}(23)-\mathrm{C}(24)-\mathrm{H}(24 \mathrm{~A})$ & 119.7 \\
$\mathrm{C}(24)-\mathrm{C}(25)-\mathrm{C}(26)$ & $120.1(2)$ \\
$\mathrm{C}(24)-\mathrm{C}(25)-\mathrm{H}(25 \mathrm{~A})$ & 119.9 \\
$\mathrm{C}(26)-\mathrm{C}(25)-\mathrm{H}(25 \mathrm{~A})$ & 119.9 \\
$\mathrm{C}(27)-\mathrm{C}(26)-\mathrm{C}(25)$ & $119.6(2)$ \\
$\mathrm{C}(27)-\mathrm{C}(26)-\mathrm{H}(26 \mathrm{~A})$ & 120.2 \\
$\mathrm{C}(25)-\mathrm{C}(26)-\mathrm{H}(26 \mathrm{~A})$ & 120.2 \\
$\mathrm{C}(26)-\mathrm{C}(27)-\mathrm{C}(28)$ & $120.5(2)$ \\
$\mathrm{C}(26)-\mathrm{C}(27)-\mathrm{H}(27 \mathrm{~A})$ & 119.8 \\
$\mathrm{C}(28)-\mathrm{C}(27)-\mathrm{H}(27 \mathrm{~A})$ & 119.8 \\
$\mathrm{C}(23)-\mathrm{C}(28)-\mathrm{C}(27)$ & $120.4(2)$ \\
$\mathrm{C}(23)-\mathrm{C}(28)-\mathrm{H}(28 \mathrm{~A})$ & 119.8 \\
$\mathrm{C}(27)-\mathrm{C}(28)-\mathrm{H}(28 \mathrm{~A})$ & \\
\hline & 119.8 \\
& \\
\hline
\end{tabular}

Symmetry transformations used to generate equivalent atoms: 
Table 4. Anisotropic displacement parameters $\left(\AA^{2} \times 10^{3}\right)$ for paulo4. The anisotropic displacement factor exponent takes the form: $-2 \pi^{2}\left[h^{2} a * 2 U^{11}+\ldots+2 h k a * b * U^{12}\right]$

\begin{tabular}{|c|c|c|c|c|c|c|}
\hline & $\mathrm{U}^{11}$ & $U^{22}$ & $\mathrm{U}^{33}$ & $\mathrm{U}^{23}$ & $\mathrm{U}^{13}$ & $\mathrm{U}^{12}$ \\
\hline $\operatorname{Br}(1)$ & $37(1)$ & $37(1)$ & $34(1)$ & 11(1) & $6(1)$ & $-15(1)$ \\
\hline $\operatorname{Si}(1)$ & $20(1)$ & 19(1) & $20(1)$ & $-3(1)$ & $-2(1)$ & $-1(1)$ \\
\hline $\mathrm{N}(1)$ & $19(1)$ & $15(1)$ & $14(1)$ & $-1(1)$ & $2(1)$ & $2(1)$ \\
\hline $\mathrm{C}(1)$ & $20(1)$ & $18(1)$ & $16(1)$ & $-3(1)$ & $2(1)$ & 1(1) \\
\hline$C(2)$ & $18(1)$ & $22(1)$ & $18(1)$ & $-5(1)$ & 1(1) & 1(1) \\
\hline$C(3)$ & $18(1)$ & $23(1)$ & $20(1)$ & $-4(1)$ & $2(1)$ & $0(1)$ \\
\hline$C(4)$ & $23(1)$ & $20(1)$ & $19(1)$ & $-2(1)$ & $3(1)$ & $2(1)$ \\
\hline$C(5)$ & $27(1)$ & $22(1)$ & $20(1)$ & $-3(1)$ & $3(1)$ & $0(1)$ \\
\hline$C(6)$ & $18(1)$ & $19(1)$ & $17(1)$ & $-4(1)$ & 1(1) & $-4(1)$ \\
\hline$C(7)$ & $18(1)$ & $24(1)$ & $22(1)$ & $-3(1)$ & $-1(1)$ & $2(1)$ \\
\hline$C(8)$ & $23(1)$ & $20(1)$ & $26(1)$ & $0(1)$ & $-6(1)$ & $0(1)$ \\
\hline$C(9)$ & $25(1)$ & 21(1) & $19(1)$ & $0(1)$ & 1(1) & $-9(1)$ \\
\hline$C(10)$ & $22(1)$ & $24(1)$ & $26(1)$ & $-1(1)$ & $7(1)$ & $0(1)$ \\
\hline$C(11)$ & $23(1)$ & $19(1)$ & $26(1)$ & $1(1)$ & $5(1)$ & $2(1)$ \\
\hline$C(12)$ & $28(1)$ & $40(1)$ & $34(1)$ & $7(1)$ & $4(1)$ & $-4(1)$ \\
\hline$C(13)$ & $28(1)$ & $28(1)$ & $39(1)$ & $-12(1)$ & $-3(1)$ & $2(1)$ \\
\hline$C(14)$ & $39(1)$ & $35(1)$ & $30(1)$ & $3(1)$ & $-11(1)$ & $2(1)$ \\
\hline$C(15)$ & $18(1)$ & $19(1)$ & $18(1)$ & $-1(1)$ & $-2(1)$ & 1(1) \\
\hline$C(16)$ & $19(1)$ & $17(1)$ & $19(1)$ & $0(1)$ & $4(1)$ & $0(1)$ \\
\hline$C(17)$ & $18(1)$ & $16(1)$ & $16(1)$ & $2(1)$ & $-1(1)$ & $3(1)$ \\
\hline$C(18)$ & $23(1)$ & $24(1)$ & $26(1)$ & $6(1)$ & $7(1)$ & $5(1)$ \\
\hline$C(19)$ & $36(1)$ & $39(1)$ & $25(1)$ & $1(1)$ & $5(1)$ & $21(1)$ \\
\hline$C(20)$ & $44(1)$ & $34(1)$ & $34(1)$ & $-18(1)$ & $-16(1)$ & $23(1)$ \\
\hline$C(21)$ & $28(1)$ & 21(1) & $51(2)$ & $-8(1)$ & $-16(1)$ & $5(1)$ \\
\hline$C(22)$ & $17(1)$ & $20(1)$ & $32(1)$ & $1(1)$ & $-1(1)$ & $2(1)$ \\
\hline$C(23)$ & $22(1)$ & $18(1)$ & $14(1)$ & $2(1)$ & $5(1)$ & $0(1)$ \\
\hline$C(24)$ & $27(1)$ & $18(1)$ & $16(1)$ & $2(1)$ & $5(1)$ & $-2(1)$ \\
\hline$C(25)$ & $24(1)$ & $34(1)$ & $17(1)$ & $2(1)$ & 1(1) & $-6(1)$ \\
\hline$C(26)$ & $24(1)$ & $38(1)$ & $22(1)$ & $9(1)$ & $2(1)$ & $8(1)$ \\
\hline$C(27)$ & $39(1)$ & $22(1)$ & $31(1)$ & $4(1)$ & $4(1)$ & $9(1)$ \\
\hline$C(28)$ & $30(1)$ & $19(1)$ & $27(1)$ & $0(1)$ & $0(1)$ & $0(1)$ \\
\hline
\end{tabular}


Table 5. Hydrogen coordinates ( $\left.\mathrm{x} 10^{4}\right)$ and isotropic displacement parameters $\left(\AA^{2} \times 10^{3}\right)$ for paulo4.

\begin{tabular}{|c|c|c|c|c|}
\hline & $\mathrm{x}$ & $\mathrm{y}$ & z & $\mathrm{U}(\mathrm{eq})$ \\
\hline $\mathrm{H}(1 \mathrm{~A})$ & 6291 & 11819 & -2821 & 21 \\
\hline $\mathrm{H}(7 \mathrm{~A})$ & 7191 & 7858 & -1059 & 26 \\
\hline $\mathrm{H}(8 \mathrm{~A})$ & 5462 & 6752 & -259 & 29 \\
\hline $\mathrm{H}(10 \mathrm{~A})$ & 290 & 9371 & -370 & 29 \\
\hline $\mathrm{H}(11 \mathrm{~A})$ & 2033 & 10481 & -1167 & 27 \\
\hline $\mathrm{H}(12 \mathrm{~A})$ & 13062 & 15362 & -2898 & 51 \\
\hline $\mathrm{H}(12 \mathrm{~B})$ & 15068 & 14416 & -2630 & 51 \\
\hline $\mathrm{H}(12 \mathrm{C})$ & 14990 & 15883 & -2385 & 51 \\
\hline $\mathrm{H}(13 \mathrm{~A})$ & 9431 & 15646 & -1323 & 48 \\
\hline $\mathrm{H}(13 \mathrm{~B})$ & 9401 & 16209 & -2029 & 48 \\
\hline $\mathrm{H}(13 \mathrm{C})$ & 11297 & 16690 & -1497 & 48 \\
\hline $\mathrm{H}(14 \mathrm{~A})$ & 13200 & 13819 & -831 & 53 \\
\hline $\mathrm{H}(14 \mathrm{~B})$ & 15211 & 14742 & -1032 & 53 \\
\hline $\mathrm{H}(14 \mathrm{C})$ & 15021 & 13286 & -1292 & 53 \\
\hline $\mathrm{H}(15 \mathrm{~A})$ & 10124 & 9389 & -2357 & 22 \\
\hline $\mathrm{H}(15 \mathrm{~B})$ & 11887 & 10189 & -2750 & 22 \\
\hline $\mathrm{H}(16 \mathrm{~A})$ & 10075 & 10627 & -3891 & 22 \\
\hline $\mathrm{H}(16 \mathrm{~B})$ & 10316 & 11920 & -3471 & 22 \\
\hline $\mathrm{H}(18 \mathrm{~A})$ & 13858 & 8949 & -3501 & 28 \\
\hline $\mathrm{H}(19 \mathrm{~A})$ & 13993 & 7242 & -4226 & 40 \\
\hline $\mathrm{H}(20 \mathrm{~A})$ & 10988 & 5782 & -4356 & 46 \\
\hline $\mathrm{H}(21 \mathrm{~A})$ & 7818 & 6008 & -3761 & 42 \\
\hline $\mathrm{H}(22 \mathrm{~A})$ & 7632 & 7724 & -3055 & 28 \\
\hline $\mathrm{H}(24 \mathrm{~A})$ & 5951 & 10064 & -4341 & 24 \\
\hline $\mathrm{H}(25 \mathrm{~A})$ & 2689 & 10909 & -4891 & 30 \\
\hline $\mathrm{H}(26 \mathrm{~A})$ & 1965 & 13141 & -4871 & 34 \\
\hline $\mathrm{H}(27 \mathrm{~A})$ & 4546 & 14517 & -4315 & 37 \\
\hline $\mathrm{H}(28 \mathrm{~A})$ & 7821 & 13678 & -3763 & 30 \\
\hline
\end{tabular}

